Bulletin of the Natural History Museum, 2014, 7: 93-127.

Received 21 Jul 2014; Accepted 30 Aug 2014.

DOI: $10.5937 /$ bnhmb1407093Z

UDC: $582.684 .1 .082 .5(497.11)$

\title{
REVISION OF THE GENUS HYPERICUM L. (HYPERICACEAE) IN THREE HERBARIUM COLLECTIONS FROM SERBIA
}

\author{
BOJAN ZlATKOVIĆ ${ }^{1}$, MARKO NiKOLIĆ ${ }^{1}$, MilJANA DRNDAREVIĆ ${ }^{2}$, \\ MirosLAV JOVANOVIĆ ${ }^{3}$, MARJAN NIKETIĆ ${ }^{3}$ \\ ${ }^{1}$ University of Niš, Faculty of Sciences and Mathematics, Department of Biology \\ and Ecology, Višegradska 33, 18000 Niš, Serbia, e-mail: bojanzlat@yahoo.com, \\ marko.nikolic@pmf.edu.rs \\ ${ }^{2}$ Biological Society "Dr Sava Petrović”, Višegradska 33, 18000 Niš, Serbia, \\ e-mail: miljana638@gmail.com \\ ${ }^{3}$ Natural History Museum, Njegoševa 51, 11000 Belgrade, Serbia, \\ e-mail: mniketic@nhmbeo.rs
}

This paper provides information on herbarium specimens of the genus Hypericum represented in several herbaria from Serbia. The reviewed collections include: Herbarium of Natural History Museum in Belgrade (BEO), Herbarium of the Institute of Botany and Botanical Garden "Jevremovac", University of Belgrade (BEOU), and Herbarium of the Faculty of Sciences and Mathematics, Department of Biology and Ecology, University of Niš (HMN). Total number of 1108 herbarium sheets was examined, including 426 specimens stored in BEO, 484 in BEOU and 198 in HMN. The review of revised herbarium data for 18 plant species of the genus represented in the flora of Serbia is presented. Their distribution in Serbia is reconsidered according to obtained herbarium data and shown in the maps.

Key words: Hypericum, herbarium material, revision, Serbia, distribution. 


\section{INTRODUCTION}

Hypericum L. is the type and the largest genus of the family Hypericaceae, including 420-470 species, mostly herbaceous plants, shrubs, or rarely small trees or annual species classified into 30-36 sections according to the most recent reviews (Robson 1977, Stevens 2007, Crockett \& Robson 2011). The majority of the species in this genus has general distribution in the temperate regions, while a number of them also inhabit mountain regions of the tropics, and they have been introduced to almost all continents of the World (Takhtajan 2009). According to the "Flora Europaea" (Robson 1968) 61 species and 18 sections of the genus are recorded for the continent. Although the genus Hypericum is cosmopolitan, the main centers of diversity are the Eurasian region (about 230 species) and South America, with a few additional, less important centers of diversity. Many Hypericum species are important herbs with potential value in traditional medicine of different nations, while $H$. perforatum $\mathrm{L}$. is one of the best known medicinal plants of the genus (Crockett \& Robson 2011).

Stjepanović-Veseličić $(1972,1977)$ performed the first comprehensive study of the genus Hypericum in Serbia, proving the basic information on the number of taxa and their distribution in the country. According to the updated delimitation of the genus given by Robson (1977), the native representatives from the flora of Serbia may be classified into eight sections of Hypericum: H. sect. Adenosepalum includes 2 species (Hypericum annulatum Moris and Hypericum montanum L.); H. sect. Androsaemum (Duhamel) includes only Hypericum androsaemum L.; H. sect. Drosocarpium Spach includes 6 species (Hypericum barbatum Jacq., Hypericum montbretii Spach, Hypericum richeri Vill. (Boiss.) Nyman, Hypericum rochelii Griseb. \& Schenk, Hypericum rumeliacum Boiss. and Hypericum umbellatum A. Kerner); $H$. sect. Hirtella Stef. includes one species (Hypericum hyssopifolium Chaix); $H$. sect. Hypericum includes 4 species (Hypericum elegans Stephan ex Willd, Hypericum maculatum Crantz (including 2 subspecies), Hypericum perforatum L. (including 3 subspecies) and Hypericum tetrapterum Fries); H. sect. Oligostema (Boiss.) Stef. includes one species (Hypericum humifusum L.); H. sect. Olympia (Spach) Nyman includes one species (Hypericum olympicum L.); and $H$. sect. Taeniocarpium Jaub. \& Spach includes 2 species (Hypericum hirsutum L. and Hypericum linarioides Bosse).

All enumerated species were recorded in Stjepanović-Veseličić (1972), excluding Hypericum montbretii which was reported recently as a new species in Serbian flora by Zlatković et al. (2009). On the other hand, some of the taxa and names enumerated in Stjepanović-Veseličić (1972) were erroneously reported, or their presence in Serbia was doubtful. 
In the framework of this review, based on revision and inspection of material in herbarium collections of BEO, BEOU and HMN, the majority of specimens of Hypericum taxa from different regions of Serbia were re-examined. It is recognized that herbarium data may provide high-quality information which is useful and accurate for purposes of plant checklists and offering precise data on species distribution. Therefore the main purpose of the present study was to determine presence of all Hypericum species collected in the territory of Serbia that would be useful as an addition to the forthcoming volumes of the "Flora of Serbia", offering precise floristic data for their distribution in different regions of the country.

\section{MATERIAL AND METHODS}

Voucher specimens represented in the review of the genus Hypericum are preserved in Herbarium of the Natural History Museum in Belgrade (BEO), Herbarium of the Institute of Botany and Botanical Garden "Jevremovac", University of Belgrade (BEOU), (Thiers 2014; http:// sweetgum.nybg.org/ih, continuously updated) and Herbarium of Faculty of Sciences and Mathematics, University of Niš (HMN). The separate subcollections of BEOU include: General Collection - BEOU, Collection of the Department of Plant Ecology and Geography - BEOU-KEGB and the Collection by Josif Pančić „Herbarium Pancicianum“ - BEOU (Herb Panc). The catalogue of the species in genus Hypericum was prepared exclusively by citing material collected from their native habitats in Serbia.

The revision and determination of unidentified herbarium material of genus Hypericum were conducted according to Flora Europaea (Robson 1968), but also by exploiting the regional floristic monographs relevant for the genus (Jordanov \& Kožuharov 1970, Stjepanovic-Veseličić 1972, Micevski 1995). Nomenclature is aligned with the Euro+Med (http:// ww2.bgbm.org/EuroPlusMed/) databases and adequate volumes of the Flora Europaea (Robson 1968).

Information on distribution was also collected for each Hypericum taxa and mapped in the grid system with precision of $10 \mathrm{~km}^{2}$ (Serbia, Zone 34T), based on UTM projection (http://www.luomus.fi/english/ botany/ afe/map/utm.htm; Lampinen 2001). The distribution depicted in maps shows less precise records, related to wider localities that overlap two or more UTM squares marked with sign. Uncertain locations or doubtful records were marked with a question mark (?). 


\section{Catalogue of herbarium specimens of the genus Hypericum from Serbia}

\section{Hypericum L.}

\section{H. sect. ANDROSAEMUM (Duhamel) Gordon}

\section{Hypericum androsaemum L. (Fig. 1)}

NW Serbia Boranja: Sakar, Trešnjica (Radakovića česma), UTM CQ50 (leg. Jurišić, Ž., 02-Jun-1912, 8592 BEO, sub Androsaemum officinale), rev. B., Zlatković 12-Dec-1013); Cer, UTM CQ74, CQ83, bukova šuma (leg. Stevanović, $V$. 4.1988, 45209 BEOU); (leg. Petrović, R. 1882, 42744 BEOU, sub Androsaemum vulgare, rev. B., Zlatković 12-Dec-1013); Dobre vode, UTM CQ74, 42710 BEOU); Gučevo: Crni vrh - Velika ravan, UTM CQ52 (leg. Niketić, M., Tomović, G. 25Apr-2008, 26706 BEOU-KEGB); Krupanj: Vukolovci, UTM CQ61 (leg. Jurišić, Ž. 06-Jun-1912, 8591 BEO, sub Androsaemum officinale), rev. B., Zlatković 12-Dec-1013).

\section{H. sect. HYPERICUM}

\section{Hypericum elegans Stephan} ex Willd. (Fig. 2)

Banat Deliblatska peščara: Domacija?, 25Jun-1944, 8702, 8704 BEO); (leg. Soška, Th. 25Jun-1944, det. Th., Soška, 8706 BEO); Dubovac, UTM EQ15 (leg. Pančić, J., det. J., Pančić, 3362 BEOU (Herb. Panc.)); Kajtasovo, UTM EQ26, 14-Jul-1940, 8705 BEO); 04-Jul-1943, 8703 BEO); (leg. Soška, Th. 04-Jul-1943, det. Th., Soška, 8707 BEO); C Serbia Niš: Lalinačka slatina, UTM EN59, $180 \mathrm{~m}$, les, stepolika staništa (leg. Zlatković, B., 2010, det. B., Zlatković, $7258 \mathrm{HMN}$ ); (leg. Ranđelović,V. 2003, det. V., Ranđelović, 633 HMN); Prokuplje: Dobrič, UTM EN59 (leg. Pančić, J., det. J., Pančić, 3364, 3365 BEOU (Herb. Panc.)), 14605 BEOU (Herb. Panc.); sub H. pulchellum, rev. B., Zlatković 06-Feb-2014); E Serbia Niš, UTM EN79 (leg. Pančić, J., det. J., Pančić, 3388 BEOU (Herb. Panc.), sub H. pulchellum, rev. B., Zlatković 06-Feb-2014).

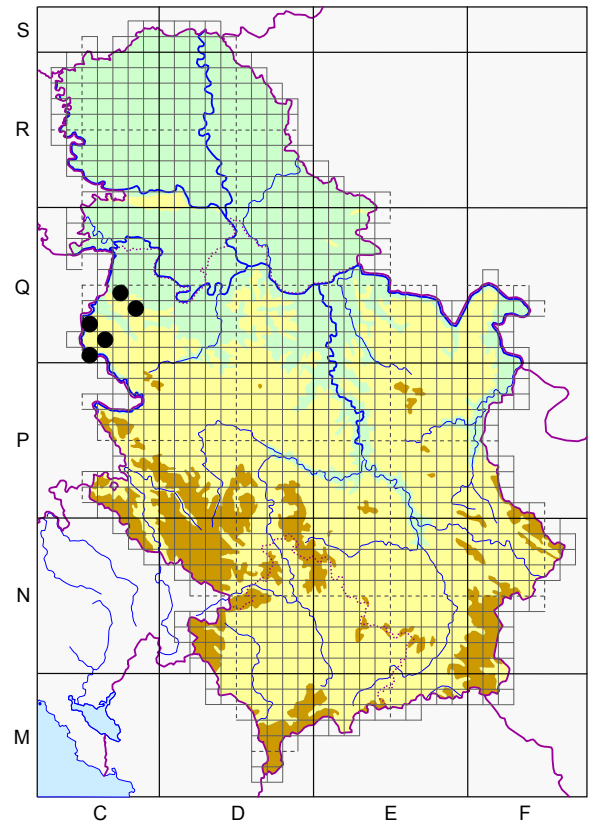

Fig. 1. - Distribution of the species $H$. androsaemum L. in Serbia.

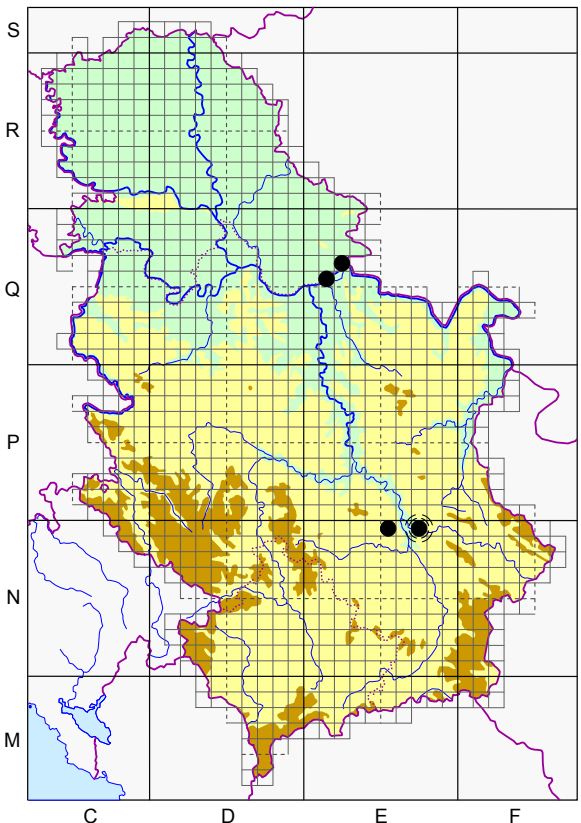

Fig. 2. - Distribution of the species H. elegans Stephan ex Willd. in Serbia. 


\section{Hypericum maculatum Crantz subsp. maculatum (Fig. 3)}

NW Serbia Suvobor: Ba, Čardak, UTM DP39, granit (leg. Jovanović, M., Niketić, M., 07May-2004, det. M., Jovanović, 43346 BEO); NE Serbia Homolje: Vukan, Veliki Vukan, UTM EQ40, $740 \mathrm{~m}$, krečnjak, stene (leg. Niketić, M., Tomović, G. 29-Apr-2003, 16460 BEOU-KEGB); Mlava - klisura: manastir Gornjak, UTM EQ40, pored puteva (leg. Jovanović, M. 18-May-2004, det. M., Jovanović, 43366 BEO); W Serbia Tara, UTM CP76, CP75, serpentinit, opožarena šumska staništa (leg. Stevanović, V., Jovanović, S., Vukojičić, S., 10-Jul-2000, 14376 BEOU-KEGB); C Serbia Kopaonik (leg. Pančić, J., det. J., Pančić, 3391, 3395 BEOU (Herb. Panc.), sub H. quadrangulum, rev. B., Zlatković 06-Feb-2014); Gobelja, UTM DN89 (leg. Rudski, I. 12-Jul-1938, det. M., Jovanović, 43340 BEO); Karaman, UTM DN89 (leg. Rudski, I. 09-Jul-1938, det. M., Jovanović, 43339 BEO); Rudnička rekadolina, UTM DN79, hrastova šuma (leg. Rajevski, L. 14-Jun-1952, 42888 BEOU); Sokolovica: Pupavičke livade, UTM EN26, 700 $\mathrm{m}$, andezit, brdske livade, 1507 BEOU); (leg. Tomović, G., Tomović, M. 25-Jun-1998, 1507 BEOU-KEGB); E Serbia Stara planina (leg. Matejić, J., 2004, det. V., Ranđelović, 3823

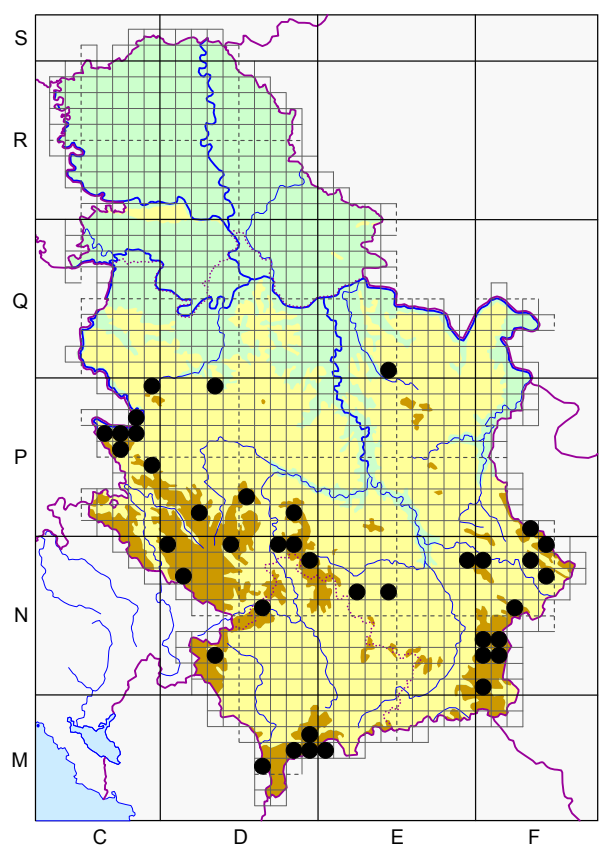

Fig. 3. - Distribution of the species H. maculatum Crantz in Serbia.

HMN); (leg. Pančić, J., det. J., Pančić, 3396 BEOU (Herb. Panc.), sub H. quadrangulum, rev. B., Zlatković 06-Feb-2014); Arbinje, UTM FN49 (leg. Tomović, G., Zlatković, B. 27-Jul-1998, 11645 BEOUKEGB); Babin zub, UTM FP30, crveni peščari i konglomerati, planinski pašnjaci (leg. Mihailović, $T$. 21-Jul-1993, det. T., Mihailović, 474 BEOU-KEGB); Dojkinci, Vrelo, UTM FN47, 1550 m, crveni peščari i konglomerati, tresava (leg. Mihailović, T. 28-Jul-1993, det. T., Mihailović, 475 BEOU-KEGB); 1550 m, crveni peščari i konglomerati, bukova šuma (leg. Mihailović, T. 28-Jul-1993, det. T., Mihailović, 476 BEOU-KEGB); Dupljak, UTM FP30, granit, visokoplaninski pašnjak (leg. Mihailović, T. 25-Jul1993, det. T., Mihailović, 477 BEOU-KEGB); Midžor, UTM FP30, crveni peščari i konglomerati, visokoplaninski pašnjak (leg. Mihailović, T. 29-Jul-1993, det. T., Mihailović, 478 BEOU-KEGB); Topli Do, Belan, UTM FP30, 1200 m (leg. Grebenščikov, O. 05-Jul-1946, det. O., Grebenščikov, 8675 BEO, sub H. maculatum subsp. quadrangulum, rev. B., Zlatković 31-Mar-2014); Tupanar, UTM FP30, crveni peščari i konglomerati, planinski pašnjaci (leg. Mihailović, T. 25-Jul-1993, det. T., Mihailović, 479 BEOU-KEGB); Žarkova čuka, UTM FP30, crveni peščari i konglomerati, planinski pašnjaci (leg. Mihailović, T. 24-Jul-1993, det. T., Mihailović, 473 BEOU-KEGB); Suva planina: Devojački grob, UTM EN98, 1400 m, krečnjak, suvi pašnjaci (leg. Bogosavljević, S. 2013, det. B., Zlatković, 7261 HMN); Vidlič: Basarski kamik, planinarski dom, UTM FN38, pored puteva (leg. Marković, M. 21-Aug-2005, det. B., Zlatković, $1725 \mathrm{HMN}$ ); Vazganica, UTM FN38, opožarena mesta, bukove šume (leg. Marković, M. 06-Jul-2010, det. M., Marković, $10311 \mathrm{HMN}$ ); pored puteva (leg. Marković, M. 25-Aug-2005, det. B., Zlatković, $1692 \mathrm{HMN}$ ); opožarena mesta, bukove šume (leg. Marković, M. 26-Aug-2008, det. M., Marković, 5201, 5261 HMN); Visočka Ržana, UTM FN47, pored puteva (leg. Marković, M. 21-Aug2005, det. M., Marković, 1712 HMN); SW Serbia Pešter: Giljeva, UTM DN17, 1300 m, krečnjak, pašnjaci, kamenjari (leg. Jovanović, S., 23-Jun-1996, 2647 BEOU-KEGB); S Serbia Radan: Dobre vode, planinarski dom, UTM EN46, obala potoka (leg. Jovanović, V., 1976, det. V., Jovanović, 5601 HMN); SE Serbia Besna Kobila, UTM FN00 (leg. Jovanović, V., 1976, det. B., Zlatković, 4422 HMN); Vlasina, brdske livade (leg. Marković, M. 2005, det. V., Ranđelović, 720 HMN); Kosovo Šarplanina (leg. Stevanović, V., Jovanović, S., 21-Jul-1989, 3308 BEOU-KEGB); Piribeg, UTM EM06, 
2000 m (leg. Stevanović, V. 07-Jul-1979, 2126 BEOU-KEGB); Metohija Mokra planina, UTM DN65 (leg. Rudski, I., 16-Jul-1932, det. M., Jovanović, 43337 BEO).

\section{Hypericum maculatum subsp. immaculatum (Murb.) A. Frohl (Fig. 3)}

NW Serbia Jablanik, UTM CP99, 1274 m, livada (leg. Nikolić, V., Diklić, N., 24-Jul-1954, det. V., Nikolić, 43491 BEO, sub H. perforatum, rev. B., Zlatković 01-Apr-2014); (leg. Pančić, J., det. J., Pančić, 3392 BEOU (Herb. Panc.), sub H. quadrangulum, rev. B., Zlatković 06-Feb-2014); Brezak, UTM CP94, livada (leg. Nikolić, V., Diklić, N. 28-Jun-1954, det. V., Nikolić, 43475 BEO, sub H. perforatum subsp. vulgare, rev. B., Zlatković 01-Apr-2014); W Serbia Čemerno, UTM DP52 (leg. Pančić, J., det. J., Pančić, 3393 BEOU (Herb. Panc.), sub H. quadrangulum, rev. B., Zlatković 06-Feb-2014); Javor: Kušići, UTM DP21, livade (leg. Nikolić, V., Mladenović, S. 15-Jul-1982, det. M., Jovanović, 43352 BEO); Tara: Jagoštica, UTM CP66 (leg. Jovanović, S., Jovanović, B. 05-Aug-2004, 22064 BEOUKEGB); Kaluđerske bare, UTM CP86, $1048 \mathrm{~m}$, bukovo-grabova šuma (leg. Jovanović, M. 18-Sep2003, det. M., Jovanović, 43367 BEO); Ljuto polje - Karaića bare, UTM CP75, tresave i Nardetum strictae (leg. Stevanović, V., Jovanović, S., Vukojičić, S. 08-Jul-2000, 14609 BEOU-KEGB); Rača, UTM CP87, livade (leg. Diklić, N., Rakin, M. 17-Jul-1963, det. N., Diklić, 43359 BEO); Šljivovica, UTM CP86 (leg. Stevanović, V., Vukojičić, S. 30-Aug-2000, 14480 BEOU-KEGB); Zmajevski potok, UTM CP75, šume, Picea omorika (leg. Stevanović, V., Jovanović, S., Vukojičić, S. 09-Jul-2000, 14701, 14720 BEOUKEGB); Zvezda: Veliki Stolac, UTM CP75 (leg. Menković, N. 21-Jul-2000, 10668 BEOU-KEGB); C Serbia Kopaonik (leg. Pančić, J., det. J., Pančić, 3390, 3394 BEOU (Herb. Panc.), sub H. quadrangulum, rev. B., Zlatković 06-Feb-2014); Bele stene, UTM DN89, krečnjak (leg. Kuzmanović, N., Jakovljević, K. Buzurović, I. 06-Aug-2011, 36104 BEOU-KEGB); (leg. Rudski, I. 12-Jul-1938, 8666 BEO, sub. H. maculatum, rev. B., Zlatković 31-Mar-2014); Crni vrh, UTM DN98, $900 \mathrm{~m}$, silikat, šume, Fagetum montanum (leg. Lakušić, D. 25-Jun-1996, 2035 BEOU-KEGB); Gobelja, UTM DN89, stene (leg. Rudski, I. 12-Jul-1938, det. M., Jovanović, 43342 BEO, sub. H. maculatum, rev. B., Zlatković 31Mar-2014); Kadijevac, kamp, UTM DN79, 1450 m, granit, vlažne livade (leg. Laušević, R. 10-Sep1988, det. D., Lakušić, BEOU-KEGB); (leg. Stanić, S. 13-Jul-1988, det. D., Lakušić, BEOU-KEGB); Karaman, UTM DN89 (leg. Rudski, I. 09-Jul-1938, det. M., Jovanović, 43341 BEO, sub. H. maculatum, rev. B., Zlatković 31-Mar-2014); Kukavica, UTM DN79, 1600 m, livade i pašnjaci (leg. Lakušić, D., Niketić, M. 10-Aug-1986, det. D., Lakušić, BEOU-KEGB); Mali Karaman, UTM DN89, $1850 \mathrm{~m}$, granit, ass. Vaccinio-Juniperetum sibiricae (leg. Laušević, R. 10-Sep-1988, det. D., Lakušić, BEOUKEGB); Milanov vrh, UTM DN89 (leg. Rudski, I. 08-Jul-1938, 8669 BEO, sub. H. maculatum, rev. B., Zlatković 31-Mar-2014); Oštre stene, UTM DN79, 1700 m, krečnjak, ass. Piceetum excelsae (leg. Stevanović, V., Niketić, M. 04-Jun-1985, det. D., Lakušić, BEOU-KEGB); Samokovska reka - klisura, UTM DN89, 1400 m, granit (leg. Laušević, R. 9.1988, det. D., Lakušić, BEOU-KEGB); 1400 m, ass. Piceetum excelsae (leg. Stanišić, S. 14-Jul-1988, det. D., Lakušić, BEOU-KEGB); Srebrnac, UTM DN89, 10-Jul-2004, 16056 BEOU-KEGB); pašnjaci, kamenjari (leg. Diklić, N., Mladenović, S. 17-Jul-1985, det. M., Jovanović, 43356 BEO); Suvo rudište, UTM DN89, 1800-1900 m (leg. Nikolić, V. 29-Aug1982, det. M., Jovanović, 43333 BEO, sub. H. maculatum, rev. B., Zlatković 31-Mar-2014); Željin, UTM DP81, pašnjak (leg. Adamović, Ž. 29-Sep-1966, det. Ž., Adamović, 43336 BEO, sub. H. maculatum, rev. B., Zlatković 31-Mar-2014); E Serbia Jerma - klisura: Zvonačka Banja, Cvetkova livada, UTM FN25 (leg. Nikolić, V., Diklić, N., 14-Jul-1965, det. N., Diklić, 43362 BEO); Stara planina: Babin zub, UTM FP30, 1689 m, silikat, ass. Rubeto-Juniperetum intermediae (leg. Lakušić, D., Lakušić, B. 19Jul-2012, 34951 BEOU-KEGB); visokoplaninski pašnjak (leg. Ranđelović, V. 1998, det. V., Ranđelović, 1406 HMN); Suva planina: Devojački grob - Trem, UTM EN98, krečnjak (leg. Vukojičić, S., Tomović, G. 08-Jul-1997, 6307 BEOU-KEGB); Kosmovac, UTM EN98 (leg. Pelivanović, S., 43084 BEOU); Trem - Golemo stražište - Valožje, UTM FN08, 1600 m, krečnjak (leg. Gusev, CH., Zlatković, B. 07-Jul-1997, 6452 BEOU-KEGB); SW Serbia Golija, UTM DN49, proplanci (leg. Janković, M., 18-Aug-1949, 42916 BEOU); Zlatar: Vodena poljana - Golo brdo, UTM DN09, 1200-1500 m, livade (leg. Nikolić, V., Diklić, N. 16-Jul-1971, det. N., Diklić, 43358 BEO); SE Serbia Besna Kobila, UTM FN00, 1500-1600 m, livade (leg. Nikolić, V., Diklić, N., 22-Jul-1967, det. N., Diklić, 43363 BEO); 1600-1900 m, livade i pašnjaci (leg. Nikolić, V., Diklić, N. 17-Jul-1968, det. N., Diklić, 43360 BEO); 1650-1922 m, pašnjaci, kamenjari (leg. Nikolić, V., Mladenović, S. 15-Jul-1979, 43353 BEO); Strešer: Veliki Strešer, UTM FN02, 1700-1890 m, pašnjaci (leg. Nikolić, V., Diklić, N. 23-Jul-1967, det. 
N., Diklić, 43357 BEO); Vlasina, pašnjak (leg. Gajić, M. 07-Aug-1950, 42965 BEOU); šuma molike, 19Jul-1886, 42512 BEOU); Hotel "Vlasina rid", UTM FN03, livada (leg. Nikolić, V., Diklić, N., Bogdanović, M. 25-Jul-1972, det. V., Nikolić, 43426 BEO, sub H. perforatum, rev. B., Zlatković 01-Apr-2014); Stratorija, UTM FN12 (leg. Černjavski, P. 15-Aug-1930, det. P., Černjavski, 8665 BEO, sub. H. maculatum, rev. B., Zlatković 31-Mar-2014); Vlasina rid, UTM FN03, pašnjaci, Festuco-Nardetum strictae (leg. Gudžić, B., Ranđelović, V. 20-Jul-1998, 43345 BEO, sub. H. maculatum, rev. B., Zlatković 31-Mar-2014); Vlasinsko jezero, vlažne livade (leg. Ranđelović, V. 1998, det. V., Ranđelović, 1726 HMN); Vrtop, UTM FN13, Festuco-Agrostietum subass. nardetosum (leg. Jovanović, S., Niketić, M. 29Aug-1991, 1721 BEOU-KEGB); obala potoka (leg. Černjavski, P. 16-Aug-1936, det. P., Černjavski, 8632 BEO, sub H. perforatum subsp. vulgare, rev. B., Zlatković 01-Apr-2014); Kosovo Koprivnik, UTM DN32, 1600 m (leg. Grebenščikov, O., 13-Jul-1939, det. O., Grebenščikov, 8664 BEO); Šar-planina, det. M., Jovanović, 43338 BEO, sub. H. maculatum, rev. B., Zlatković 31-Mar-2014); Careve livade, UTM DM96 (leg. Rudski, I. 06-Jul-1961, 42516 BEOU); Gine vode, UTM DM96, tresava, molikova šuma, 06-Jul-1961, 42956 BEOU); šuma molike (leg. Janković, M. 08-Jul-1961, 42940 BEOU); Ošljak Popovo prase, UTM DM97, livade (leg. Nikolić, V., Diklić, N., Mladenović, S. 24-Jul-1978, det. M., Jovanović, 43335 BEO, sub. H. maculatum, rev. B., Zlatković 31-Mar-2014); Prevalac, UTM DM96, 1600 m, livade (leg. Nikolić, V., Diklić, N. 13-Jul-1968, det. N., Diklić, 43361 BEO); Metohija Mokra planina, UTM DN65 (leg. Rudski, I., 16-Jun-1932, det. M., Jovanović, 43364 BEO); livade (leg. Rudski, I. 16-Jun-1932, det. M., Jovanović, 43365 BEO); Šar-planina: Drajčići - Crni vrh, UTM DM65, livade, pašnjaci, kamenjari (leg. Nikolić, V., Diklić, N., Mladenović, S. 18-Jul-1978, det. M., Jovanović, 43334 BEO, sub. H. maculatum, rev. B., Zlatković 31-Mar-2014); Kobilica, UTM DM86, 2400 m, pašnjaci, kamenjari (leg. Nikolić, V., Diklić, N., Mladenović, S. 19-Jul-1977, det. M., Jovanović, 43354, 43355 BEO); Kobilica - Beograd, UTM DM86, 1700 m, kalkšisti, bukova šuma (leg. Duraki, š. 04-Aug-2002, det. Š., Duraki, 973 BEOU-KEGB); Kobilica - Crn kamen, UTM DM86, $1700 \mathrm{~m}$, hlorit-sericitski škriljci, planinski pašnjaci (leg. Duraki, Š. 14-Aug-2003, det. Š., Duraki, 972 BEOU-KEGB); Kobilica Kara Nikola, UTM DM86, 2400 m, gabro, visokoplaninski pašnjak (leg. Duraki, Š. 14-Aug-2003, det. Š., Duraki, 970 BEOU-KEGB); Kobilica - Treskavac, UTM DM86, 2000 m, krečnjak, između kamenih ploča (leg. Duraki, Š. 29-Jul-2003, det. Š., Duraki, 971 BEOU-KEGB); 1950 m, krečnjak, kamenjari, pašnjaci (leg. Duraki, š. 29-Jul-2004, det. Š., Duraki, 968 BEOU-KEGB, sub $H$. acutum, rev. B., Zlatković 06-Feb-2014).

\section{Hypericum perforatum L. subsp. perforatum (Fig. 4)}

Bačka Novi Sad, UTM DR01 (leg. Šainović, B., 31-Aug-1966, 42919 BEOU); Banat Deliblatska peščara (leg. Grebenščikov, O., 21Jun-1946, det. M., Jovanović, 43516 BEO); Čardak - Pojilo, UTM EQ06, peskovita mesta, sveza Festucion rupicolae (leg. Stevanović, V., Jovanović, S., Lakušić, D., Vukojičić, S., Tomović, G. 30-Sep-1997, 7593 BEOU-KEGB); Dalovo Kravak - Sušara, UTM EQ17 (leg. Sigunov, A. 01-May-1948, det. N., Diklić, 43417 BEO); Flamunda, UTM EQ07 (leg. Soška, Th. 22Jul-1943, det. Th., Soška, 8650 BEO); Vrelo, UTM DQ98 (leg. Grebenščikov, O. 21-Jun-1946, det. M., Jovanović, 43500 BEO); Kikinda, UTM DR57 (leg. Izmajlov, N. 02-Jun-1922, det. O., Grebenščikov, 8629 BEO); pored železničke pruge (leg. Izmajlov, N. 02-Jun-1922, det. N., Izmajlov, 8647 BEO); Nakovački put, UTM DR57 (leg. Sretković, G. 17-Jul-1996, 42583 BEOU); Kovin, UTM DQ95 (leg. Jovanović,

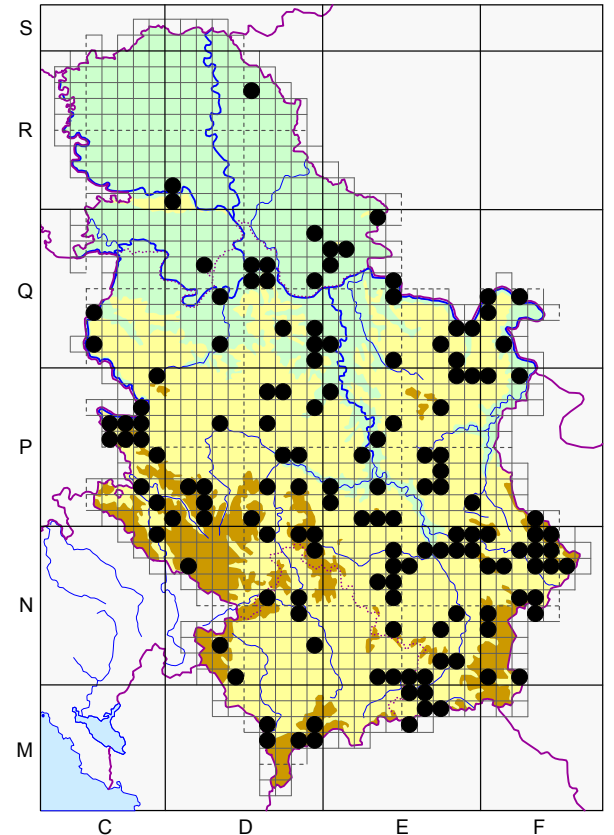

Fig. 4. - Distribution of the species H. perforatum L. in Serbia. 
E. 25-Jun-2002, 153 BEOU-KEGB); Vršačke planine, UTM EQ39 (leg. Diklić, N. 25-Jul-1957, det. N., Diklić, 43427 BEO); Široko bilo, UTM EQ39 (leg. Diklić, N. 26-Jun-1968, det. N., Diklić, 43421, 43422 BEO); Srem Fruška gora: Ledinci, Ledinačko jezero, UTM DR00, pored puteva (leg. Jovanović, M., 27-Jun-2003, det. M., Jovanović, 43526, 43449 BEO, sub. H. perforatum subsp. veronense, rev. B., Zlatković 01-Apr-2014); (leg. Jovanović, M. 27-Jun-2003, det. M., Jovanović, 43448 BEO); Ruma: Pećinci, Karlovčić, UTM DQ26, 50 m, slatina (leg. Lakušić, D. 05-Oct-1996, 2185 BEOU-KEGB); slatina (leg. Stanojević, S. 07-Jun-1996, 42575 BEOU); NW Serbia Jablanik, UTM CP99, livada (leg. Nikolić, V., 24-Jul-1954, det. V., Nikolić, N., Diklić, 43430 BEO); Loznica: Trbušnica, UTM CQ53 (leg. Mitrović, V. 14-Jun-1998, 42562 BEOU); Mali Zvornik, UTM CQ51 (leg. Grubišić, D. 01-Jul-1999, 9373 BEOUKEGB); Medvednik, UTM CP99, bukova šuma (leg. Nikolić, V., Diklić, N. 26-Jul-1954, det. V., Nikolić, 43480, 43481, 43482 BEO); Šumadija Beograd (leg. Jovanović, S., 1986, BEOU-KEGB); Ada Ciganlija, UTM DQ55 (leg. Lakušić, D. 28-Jun-2007, 24599 BEOU-KEGB); Bubanj Potok, od autopijace ka naplatnoj rampi, UTM DQ65, 114-116 m (leg. Kabaš, E., Kuzmanović, N. 17-Jul-2011, 35240 BEOUKEGB); Dedinje, UTM DQ55 (leg. Soška, Th. 12-Jul-1926, 42604 BEOU); Novi Beograd, Blok 24 (okretnica 67), UTM DQ56 (leg. Mrdaković, M. 24-Aug-1996, 42584 BEOU); kod filmske akademije, UTM DQ56 (leg. Mrdaković, M. 26-Jul-1996, 42585, 42586 BEOU); Višnjica, UTM DQ66 (leg. Bogojević, R. 1959-1963, 42917 BEOU); Zvezdara, UTM DQ65, pašnjak (leg. Lakušić, D. 06-Sep-1998, 9225 BEOU-KEGB); Borač: Borački krš, UTM DP66, gabro, stene (leg. Stevanović, V. 08-Jun-2003, 22297 BEOU-KEGB); Gornjačka klisura, UTM EQ40, stene, sipari i škrape, 26-May-1989, 45210 BEOU); Kragujevac, UTM DP97 (leg. Rudski, I. 07-Sep-1937, det. M., Jovanović, 43519 BEO); Badnjevac, UTM EP08 (leg. Rudski, I. 22-Aug-1934, 8624 BEO); Bubanj, ul. Đure Pucara, UTM DP97, 190 m, ruderalne površine (leg. Marinković, D., det. D., Marinković, BEOU-KEGB); Disino brdo, UTM DP97 (leg. Rudski, I. 04-Jul-1939, 8644 BEO); Divlje poljane, UTM DP97 (leg. Rudski, I. 24-Aug-1940, det. Th., Soška, 8656 BEO); Mladenovac: Česma Crkvenac, UTM DQ72, parkovi (leg. Anđelković, S. 13-Aug-2001, 205 BEOU-KEGB); Obrenovac: Urovci, termoelektrana (TENT B), UTM DQ34 (leg. Kostić, Z. 14-May-2000, det. S., Jovanović, BEOU-KEGB); Rudnik, UTM DP68, mešovita listopadno-četinarska šuma (leg. Jovanović, M. 10-Jun-2003, det. M., Jovanović, 43451 BEO); Pomoravlje Ćuprija: Sisevac, UTM EP46, 550 m (leg. Damjanović, D., 16-Aug-1983, det. M., Jovanović, 43498 BEO); Lajkovac, UTM DQ31, šume (leg. Černjavski, P. 06-Jul-1942, det. P., Černjavski, 8663 BEO); Les? (leg. Pančić, J., det. J., Pančić, 3381 BEOU (Herb. Panc.)); Paraćin: manastir Ravanica, UTM EP46 (leg. Pančić, J., det. J., Pančić, 3383 BEOU (Herb. Panc.)); Suvaja - klisura, UTM EP24, krečnjak, 11-Jul-1998, BEOU-KEGB); Požarevac: Požeženska peščara, UTM EQ45 (leg. Bogojević, R. 05-Jun-1987, 3707 BEOU-KEGB); Smederevska Palanka: Azanja, UTM DQ92, zapušteni voćnjaci (leg. Jovanović, M. 12-Sep-2002, det. M., Jovanović, 43508, 43459 BEO); Glibovac, UTM DQ91 (leg. Tomić, A. 16-Jul-1991, 42577 BEOU); Kudreč - jezero, UTM DQ91, livada (leg. Jovanović, M. 03-Jul-2003, det. M., Jovanović, 43455 BEO); Mala Plana, UTM EQ01, šuma (leg. Tomić, A. 30-Jul-1991, 42751 BEOU); Mikulja, UTM DQ91, šuma (leg. Tomić, A. 23-May-1952, 42581 BEOU); Vodice, UTM DQ90, livade (leg. Tomić, A. 25-Jul-1991, 42574 BEOU); Vidovača, UTM DQ90, šume (leg. Jovanović, M. 03-Jul-2003, det. M., Jovanović, 43457 BEO); NE Serbia Bor: Crni vrh, UTM FP09, bukova šuma, kamenjar (leg. Nikolić, V., 27-Jun-1955, det. V., Nikolić, 43447 BEO); (leg. Nikolić, V. 30-Jul-1956, det. V., Nikolić, 43472 BEO); Stol, UTM EP99, kamenjar (leg. Nikolić, V. 25-Jun-1955, det. V., Nikolić, 43446 BEO); Veliki krš, UTM EP89, šibljaci (leg. Diklić, N. 14-Jul-1959, det. N., Diklić, 43477 BEO); 850 m, šibljaci (leg. Diklić, N. 16-Jul-1959, det. N., Diklić, 43439 BEO); kamenjar (leg. Diklić, N. 15-Aug-1959, det. N., Diklić, 43505 BEO); pašnjaci, kamenjari (leg. Diklić, N. 29-Jun-1960, det. N., Diklić, 43440 BEO); šibljak jorgovana (leg. Nikolić, V., Diklić, N., Bogdanović, M. 17-Jun-1972, det. V., Nikolić, 43520 BEO, sub. H. perforatum subsp.veronense, rev. B., Zlatković 01-Apr-2014); Donji Milanovac, UTM EQ82, pored puteva (leg. Nikolić, V., Diklić, N., Rakin, M. 14-Sep-1965, det. N., Diklić, 43484 BEO); Paprenica - dolina, UTM EQ92 (leg. Diklić, N. 03-Aug-1966, det. N., Diklić, 43501 BEO); Poreč, UTM EQ80, 29-Jun-1934, det. Th., Soška, 8643 BEO); šume (leg. Nikolić, V., Diklić, N. 02-Aug-1966, det. N., Diklić, 43490 BEO); Porečka reka - dolina, UTM EQ92, obala potoka (leg. Nikolić, V., Diklić, N. 04-Aug-1966, det. N., Diklić, 43487 BEO); šuma (leg. Nikolić, V., Rakin, M. 19-Sep-1965, det. N., Diklić, 43499 BEO); Đerdap: Dobra - Kožica, UTM FQ03, pored puteva (leg. Nikolić, V., Diklić, N. 21-Sep-1967, det. N., Diklić, 43486 BEO); Golubac, UTMEQ44, 150 m(leg. Lindtner, V. 04-Jul-1946, det. M., Jovanović, 43518 BEO, sub. H. perforatum subsp. angustifolium, rev. B., Zlatković 01-Apr-2014); Mali Štrbac, UTM FQ04, 600 m, krečnjak, stene (leg. Vukojičić, S., Tomović, G. 16-Jul-2001, 13427 BEOU-KEGB); Sip, UTM FQ24, livade (leg. Nikolić, V., Diklić, N. 24-Jun-1967, det. N., Diklić,43485BEO); Kladovo, UTMFQ24,2783BEOU-KEGB); (leg. Pančić, J., det. J., Pančić, 14603 BEOU (Herb. Panc.)); Majdanpek, UTM EQ71 (leg. Pančić, J., det. J., 
Pančić, 3384 BEOU (Herb. Panc.)); Miroč: Filipovo brdo, UTM FQ03, šume (leg. Diklić, N., Pajović, M. 30-Jul-1955, det. N., Diklić, 43473 BEO); Konjska Glava, UTM FQ03, šibljaci (leg. Diklić, N., Nikolić, V. 28-Jul-1955, det. N., Diklić, 43433, 43434BEO); Rt?, UTMFQ03, livada (leg. Diklić, N. 20-Jun-1955, det. N., Diklić, 43431 BEO); Visoki čukar, UTM FQ03, šibljaci (leg. Diklić, N. 23-Jun-1955, det. N., Diklić, 43428 BEO); Negotin: Kapu Dalu?, UTM FP29 (leg. Pančić, J., det. J., Pančić, 3387 BEOU (Herb. Panc.)); Vratna - kanjon, UTMFQ11 (leg. Pančić, J., det. J., Pančić,3385BEOU(Herb. Panc.)); Zlot: Malinik, UTM EP77, 800 m, krečnjak (leg. Lakušić, D. 16-Jul-1986, 334 BEOU-KEGB); W Serbia Javor: Ciganska ravan, UTM DP20, 1400 m, krečnjak, kamenjar (leg. Tomović, G., 27-Jul-1996, 1835 BEOU-KEGB); Kušići, UTM DP21, livade (leg. Nikolić, V., Mladenović, S. 15-Jul-1982, det. N., Diklić, 43405 BEO); Kopaonik: Čajetina, UTM CP94, hrastova šuma (leg. Rajevski, L. 21-Jul-1952, 42889 BEOU); Mokra Gora: Šargan, UTM CP85, 678 m, serpentinit, kamenjar (leg. Kuzmanović, N., Batanjski, V., Vicić, D. 17-Jul-2010, 35490 BEOU-KEGB); Mučanj, UTM DP12, DP22, 1300 m (leg. Lakušić, D., Stanić, S. 23-Jan-1990, det. D., Lakušić, S., Stanić, BEOU-KEGB); krečnjak, kamenjar, Potentilleto-Festucetum valesiaceae (leg. Lakušić, D., Stanić, S. 27-Feb-1990, det. D., Lakušić, S., Stanić, BEOU-KEGB); kamenjar (leg. Nikolić, V. 02-Aug-1981, det. N., Diklić, 43400 BEO); Ovčarsko-Kablarska klisura: Ovčar Banja, Orlove stene, UTM DP36 (leg. Jovanović, M., Niketić, M. 21-Jun-2004, det. M., Jovanović, 43454 BEO); Perućac: Derventa, UTM CP66, krečnjak, pored puteva (leg. Jovanović, $M$. 19-Sep-2003, det. M., Jovanović, 43458 BEO); Tara, UTM CP76, CP75 (leg. Menković, N. 16-Jul-2000, 10686 BEOU-KEGB); Kaluđerske bare, UTM CP86, šuma, Ostrya carpinifolia (leg. Diklić, N. 30-Jun1965, det. N., Diklić, 43483 BEO); serpentinit, četinarske šume, Erico-Pinetum nigrae (leg. Jovanović, S., Jovanović, B. 06-Aug-2004, 22046 BEOU-KEGB); Perućac, UTM CP76 (leg. Diklić, N., Nikolić, V., Rakin, M. 13-Jul-1963, det. N., Diklić, 43503 BEO); Predov krst, UTM CP66 (leg. Grubišić, D. 02-Jul-1999, 9356 BEOU-KEGB); Rača, UTM CP87, šume, Ostrya carpinifolia (leg. Diklić, N., Rakin, M. 15-Jul-1963, det. N., Diklić, 43504 BEO); Stolac, UTM CP75, krečnjak (leg. Stevanović, V., Vukojičić, S., Tomović, G., Zlatković, B. 03-Aug-2003, 17378 BEOU-KEGB); C Serbia Goč: Drenjička kosa, UTM DP82, hrastove i bukove šume (leg. Delfi, B., 06-Aug-1936, 8631 BEO); Jelenac, UTM DP82 (leg. Delfi, B. 06-Aug1936, 8628 BEO); Kopaonik, 31-Jul-1952, 42914 BEOU); (leg. Popović, M., Mišić, V. 5.1952, 42904 BEOU); UTM DN89, 1570 m, bukova šuma, 18-Jul-1952, 42901 BEOU); Golo Brdo, UTM DN89, serpentinit, 12-Aug-1986, 42619 BEOU); Hajdučki potok - dolina, UTM DN89, 1500 m, ass. Piceetum excelsae (leg. Stanišić, S. 14-Jul-1988, det. D., Lakušić, BEOU-KEGB); Kozje stene, UTM DN99, kamenjar (leg. Nikolić, V., Diklić, N. 16-Jul-1960, det. N., Diklić, 43476 BEO); Mala Gobelja, UTM DN89, šuma smrče i jele, 04-Aug-1952, 42903 BEOU); Treska, UTM DN98, 1500 m, serpentinit, kamenjar (leg. Lakušić, D. 18-Jul-1988, det. D., Lakušić, BEOU-KEGB); Kraljevo, UTM DP74, livada, 21-Jul-1948, det. V., Lindtner, 8627 BEO); Čukojevac, UTM DP84 (leg. Rudski, I. 20-Jun-1938, 8658 BEO); Niš: Batušinačke bare, UTM EN68 (leg. Matejić, J. 2007, det. V., Ranđelović, 4163 HMN); Pasjača, UTM EN47, EN57, livade (leg. Nikolić, V. 14-Jul-1959, det. N., Diklić, 43408 BEO); Raška: Matovići Trnava, UTM DN69, serpentinit (leg. Vukojičić, S., Niketić, M. 21-Aug-1997, 7033 BEOU-KEGB); krečnjak (leg. Vukojičić, S., Tomović, G. 06-Jul-1997, 6522 BEOU-KEGB); Sokolovica: Vukovići Prolom Banja, UTM EN36, 600 m, andezit, brdske livade, 764 BEOU); (leg. Tomović, G. 19-Jun-1997, 764 BEOU-KEGB); Trstenik, UTM EP02, $300 \mathrm{~m}$ (leg. Spasojević, J. 21-Sep-1993, 42572 BEOU); Pečeni grob, UTM EP02, 400 m, silikat, kamenjar (leg. Spasojević, J. 12-Jul-1992, 42580 BEOU); Veliki Jastrebac, UTM EP20, EP30, šibljak, Carpinetum orientalis (leg. Diklić, N. 23-Jul-1954, det. N., Diklić, 43474 BEO); Bela stena, UTM EP30 (leg. Sigunov, A. 30-Jul-1949, det. M., Jovanović, 43468 BEO); Trešnja, UTM EP40, hrastova šuma (leg. Jovanović, M. 03-Jun-2003, det. M., Jovanović, 43450 BEO); Vidojevica, UTM EN47, 1000 m (leg. Nikolić, v. 15-Aug-1959, det. N., Diklić, 43407 BEO); UTM EN48, EN47 (leg. Jovanović, V. 1976, det. V., Jovanović, 4450 HMN); Jabučevo, UTM EN47, livada (leg. Nikolić, V. 15-Jul-1959, det. N., Diklić, 43406 BEO); E Serbia Bela Palanka: Šljivovički vrh, Stranje - Barkov del, UTM FN17, 1038 m, krečnjak, pašnjaci, kamenjari, 992 HMN); Šljivovik, UTM FN17, 843 m, krečnjak, ass. Quercetum frainetto-cerris, 989 BEOU); 843 m, krečnjak, šume, Quercetum frainetto-cerris (leg. Jušković, M. 14-Jun-2003, 989 BEOU-KEGB); Ždrelo, UTM FN17, 588 m, krečnjak, pašnjaci, kamenjari, 990 BEOU); (leg. Jušković, M., Zlatković, B. 25-Jun-2004, 990 BEOUKEGB); Gadžin Han: Grkinja, UTM EN88, livada (leg. Matejić, J. 2006, det. M., Marković, 3888 HMN); Greben, UTM FN34, FN35, šibljaci (leg. Diklić, N., Nikolić, V. 16-Jul-1965, det. N., Diklić, 43423 BEO); UTM FN35, 1100-1300 m, kamenjar (leg. Diklić, N., Nikolić, V. 17-Jul-1965, det. N., Diklić, 43445 BEO); Jerma - klisura: Zvonačka Banja, UTM FN25, kamenjar (leg. Nikolić, V., Diklić, N., Rakin, M. 15-Jul-1965, det. N., Diklić, 43441 BEO); Studena, UTM FN25, kamenjar (leg. Diklić, N., Nikolić, V. 19Jul-1965, det. N., Diklić, 43444 BEO); Knjaževac, Miljkovac, Golemo Branište, UTM EP91, 600 m, 
krečnjak, livade (leg. Đorđević, A. 2013, det. B., Zlatković, 7260 HMN); Niš: Jelašnica - klisura, UTM EN89, 400 m, krečnjak (leg. Vukojičić, S., Tomović, G. 07-Jul-1997, 6362 BEOU-KEGB); Ozren: Janior, UTM EP73, šibljak, Carpinetum orientalis subass. syringetosum (leg. Diklić, N. 20-Jun-1957, det. N., Diklić, 43436 BEO); Leskovik, UTM EP62, 1170 m (leg. Diklić, N. 26-Jul-1957, det. N., Diklić, 43437 BEO); Paraćin: Grza - klisura, UTM EP35, krečnjak, obala jezera (leg. Pejanović, V. 25-Apr2001, BEOU-KEGB); Pirot: Krupac, Krupačko blato, UTM FN37, livada (leg. Petrović, B. 2006, det. B., Zlatković, 3265 HMN); Temštica - klisura, UTM FN28 (leg. Jovanović, S., Lakušić, D. 29-Jun1991, 330 BEOU-KEGB); Visočica - klisura, UTM FN39 (leg. Cvetković, D. 20-Jul-1991, 22705 BEOU-KEGB); Rtanj, UTM EP64, EP74 (leg. Pančić, J., det. J., Pančić, 3382 BEOU (Herb. Panc.)); Šarbanovac, UTM EP73, zapuštene obradive površine (leg. Zlatković, B., Radivojević, A. 2012, det. B., Zlatković, 6878 HMN); Ruj: Zvonce, UTM FN25 (leg. Nikolić, V., Diklić, N. 14-Jul-1965, det. N., Diklić, 43492 BEO); Seličevica, UTM EN78, EN88, kamenjar (leg. Ranđelović, V. 2004, det. V., Ranđelović, 1036 HMN); Sićevačka klisura: Oblik, UTM EN99, 400-800 m, krečnjak (leg. Živković, T. 05-Jul1997, 5780 BEOU-KEGB); Ostrovica - manastir Sv. Petka, UTM EN99 (leg. Nikolić, V., Diklić, N. 25-Jun-1963, det. N., Diklić, 43489 BEO); (leg. Nikolić, V., Diklić, N., Rakin, M. 26-Jul-1964, det. V., Nikolić, 43471 BEO); Sokobanja, UTM EP73, pašnjak (leg. Diklić, N. 1960, det. N., Diklić, 43479 BEO); Devica, Oštra čuka, UTM EP72 (leg. Diklić, N. 29-Jun-1957, det. N., Diklić, 43435 BEO); Lepterija, UTM EP73 (leg. Antonijević, R. 25-Jun-1957, 42602 BEOU); Soko grad, UTM EP73, kamenjar, 26-Jun1957, det. N., Diklić, 43506 BEO); Stara planina: Babin zub, UTM FP30, crveni peščari i konglomerati, planinski pašnjaci (leg. Mihailović, T. 13-Jul-1994, det. T., Mihailović, 481 BEOU-KEGB); Dojkinci, Vrelo, UTM FN47, $1550 \mathrm{~m}$, crveni peščari i konglomerati, tresava (leg. Mihailović, T. 28-Jul1993, det. T., Mihailović, 482 BEOU-KEGB); Rakitska reka - dolina, UTM FN25, crveni peščari i konglomerati, šume bukve, hrasta i javora (leg. Mihailović, T. 26-Jul-1993, det. T., Mihailović, 483 BEOU-KEGB); Slavinja, Bele vode, UTM FN57, pašnjak (leg. Marković, M. 8.2004, det. M., Marković, 1021 HMN); Vrtibog, UTM FN49, 1440 m, krečnjak, kamenjar (leg. Niketić, M., Tomović, G., Eurović, S. 08-Jul-2012, 36364 BEOU-KEGB); Žarkova čuka, UTM FP30, $1850 \mathrm{~m}$, crveni peščari i konglomerati, stene, rudine (leg. Stevanović, V., Niketić, M., Tomović, G. 17-Jul-1998, 11544 BEOUKEGB); Suva planina (leg. Petrović, 43090 BEOU); Devojački grob, UTM EN98, $1550 \mathrm{~m}$, krečnjak, termofilni pašnjaci (leg. Bogosavljević, S. 2013, det. B., Zlatković, 7250 HMN); Gornja Koritnica, UTM FN07, 535 m, krečnjak, brdske livade, 988, 991 HMN); Gornja Studena Devojački grob, UTM EN88 (leg. Kuzmanović, N., Batanjski, V., Vicić, D. 03-Jul-2010, 36139 BEOUKEGB); Gornji Dušnik, UTM EN98 (leg. Nikolić, V., Diklić, N., Bogdanović, M. 17-Jul-1976, det. V., Nikolić, 43522 BEO, sub. H. perforatum subsp.veronense, rev. B., Zlatković 01-Apr-2014); Mosor Sokolov kamen, UTM EN98, krečnjak (leg. Vukojičić, S., Tomović, G. 06-Jul-1997, 6631 BEOU-KEGB); Svrljiške planine: Goli vrh, UTM FN09 (leg. Nikolić, V., Diklić, N. 29-Jul-1963, det. N., Diklić, 43488 BEO); Vidlič: Basarski kamik, UTM FN37, livada (leg. Marković, M. 05-Jul-2008, det. M., Marković, 5131 HMN); kamenjar (leg. Marković, M. 05-Jul-2008, det. M., Marković, 5514 HMN); livada (leg. Marković, M. 28-Jun-2008, det. M., Marković, 10876 HMN); UTM FN38, livada (leg. Blagojevic, I. 2009, det. N., Ranđelović, $6636 \mathrm{HMN}$ ); opožarena mesta, bukove šume (leg. Marković, M. 02-Aug-2010, det. M., Marković, 10344 HMN); opožarena mesta, kamenjari (leg. Marković, M. 12-Jul-2008, det. D., Pavlović, 5324 HMN); opožarena mesta, bukove šume (leg. Marković, M. 12-Jul-2008, det. D., Pavlović, 5350 HMN); opožarena mesta, kamenjari (leg. Marković, M. 21-Jun-2009, det. M., Marković, 7566 HMN); (leg. Marković, M., Dimitrijević, M., D., Marković, Lj. 02-Aug-2010, det. M., Marković, 10828 HMN); (leg. Marković, M., Dimitrijević, M., D., Marković, Lj. 23-Jul-2009, det. M., Marković, 7930 HMN); opožarena mesta, bukove šume (leg. Marković, M., Pavlović, D., Stanković, M. 12-Jul-2008, det. D., Pavlović, 7719 HMN); planinarski dom, UTM FN38, šumske progale (leg. Marković, M. 27-Jul-2008, det. D., Pavlović, 6019 HMN); livada (leg. Marković, M. 28-Jun-2008, det. M., Marković, 4635 HMN); Četvrti kilometar, UTM FN38, livada (leg. Marković, M., Pančić, S. 6.2004, det. M., Marković, 1094, 1114 HMN); Gulenovci, UTM FN47, opožarena mesta, hrastove šume (leg. Marković, M. 13-Jun-2009, det. M., Marković, 9265 HMN); kamenjar (leg. Marković, M. 13-Jun-2009, det. M., Marković, 9295 HMN); Dobrogled, UTM FN47, kamenjar (leg. Marković, M. 11-Jun-2005, det. M., Marković, 1975 HMN); Kozarica, UTM FN47, pored puteva (leg. Marković, M. 6.2005, det. M., Marković, 2087 HMN); Krajište, UTM FN38, mezofilne šume (leg. Marković, M. 07-Aug-2009, det. M., Marković, 8478 HMN); UTM FN48, opožarena mesta, bukove šume (leg. Marković, M. 13-Jun-2010, det. M., Marković, 10088 HMN); Visoka stena, UTM FN38, opožarena mesta, bukove šume (leg. Marković, M. 24-May-2009, det. M., Marković, 9809 HMN); Krupac, UTM FN37, pored puteva (leg. Marković, M. 25-Jul-2005, det. M., Marković, $1547 \mathrm{HMN}$ ); opožarena mesta, hrastove šume (leg. Marković, M., Marković, Lj., Stojanović, Lj., 
Đorđević, L. 14-Jun-2008, det. M., Marković, 4288 HMN); livada (leg. Marković, M., Marković, Lj., Stojanović, Lj., Đorđević, L. 14-Jun-2008, det. M., Marković, 4394 HMN); Vučje, UTM FN37, opožarena mesta, kamenjari (leg. Marković, M. 06-Sep-2009, det. B., Zlatković, 7509 HMN); opožarena mesta, hrastove šume (leg. Marković, M. 20-Jul-2008, det. M., Marković, 7359 HMN); Mojinci, UTM FN57, kamenjar (leg. Marković, M. 11-Jun-2005, det. M., Marković, 1919 HMN); Odorovački kamen - Golemi vrh, UTM FN47, krečnjak (leg. Vukojičić, S., Tomović, G., Zlatković, B. 14-Aug-2003, 17456 BEOU-KEGB); Paterica?, UTM FN48, kamenjari, livade (leg. Marković, M. 29-Jul-2009, det. M., Marković, 9050 HMN); Rsovci, UTM FN48, pored puteva (leg. Marković, M. 6.2005, det. M., Marković, 2128 HMN); kamenjar (leg. Marković, M. 08-Jun-2008, det. M., Marković, 3529 HMN); opožarena mesta, bukove šume (leg. Marković, M. 09-Aug-2009, det. M., Marković, 8782 HMN); (leg. Marković, M. 17-Aug-2008, det. M., Marković, 6005, 6014 HMN); livada (leg. Marković, M. 25-Jul-2010, det. M., Marković, 9962 HMN); (leg. Marković, M., Jotić, B., Miljković, M., Marković, Lj. 24-May-2009, det. M., Marković, 9931 HMN); Smilovci, UTM FN57, šuma (leg. Marković, M. 06-Jul-2008, det. M., Marković, 8185 HMN); Vazganica, UTM FN38, opožarena mesta, bukove šume (leg. Marković, M. 06-Sep-2008, det. M., Marković, 8437 HMN); (leg. Marković, M. 17-Jul-2008, det. M., Marković, 5606 HMN); (leg. Marković, M. 26-Aug-2008, det. M., Marković, $5254 \mathrm{HMN}$ ); Vazganica - Rsovci, UTM FN48, planinski pašnjaci (leg. Marković, M., Andrejić, Z. 31-Jul-2004, det. M., Marković, 1178 HMN); Visočka Ržana, UTM FN47, livada (leg. Marković, M. 08-Jun-2008, det. M., Marković, 6334 HMN); Visočki Odorovci, UTM FN47, opožarena mesta, kamenjari (leg. Marković, M. 06-Jul-2008, det. M., Marković, 4998 HMN); VIkovijski kamik, UTM FN57, kamenjar (leg. Marković, M. 13-Jun-2009, det. M., Marković, 9405 HMN); SW Serbia Golija: Studenica, UTM DP50, serpentinit, kamenjar (leg. Lakušić, D., 01-Jul-1998, 8670 BEOUKEGB); Pešter: Giljeva, UTM DN17, 1300 m, krečnjak, pašnjaci, kamenjari (leg. Jovanović, S. 23Jun-1996, 2637 BEOU-KEGB); Priboj: Crni vrh, UTM CP82 (leg. Lindtner, v. 22-Jun-1949, det. N., Diklić, 43416 BEO); S Serbia Bujanovac: Končuljska klisura, UTM EN50 (leg. Nikolić, V., 29Jun-1965, 43496 BEO); Kraljeva kuća, UTM EN60, šuma, Quercus robur (leg. Diklić, N. 21-Sep-1961, det. N., Diklić, 43429 BEO); Lučane, UTM EM59 (leg. Nikolić, V. 27-Jun-1965, 43494 BEO); Kukavica, UTM EN73, EN84 (leg. Jovanović, V. 1972, det. V., Jovanović, 5604 HMN); (leg. Jovanović, V. 1973, det. V., Jovanović, 5605 HMN); Arničko, UTM EN73 (leg. Jovanović, V. 1973, det. V., Jovanović, 5606 HMN); Preševo: Kor?, UTM EM57, pored puteva (leg. Diklić, N. 24-Sep-1961, det. N., Diklić, 43507 BEO, sub. H. perforatum subsp. angustifolium, rev. B., Zlatković 01-Apr-2014); Radan, UTM EN45, EN46, livada (leg. Jovanović, V. 1976, det. V., Jovanović, 5607 HMN); (leg. Jovanović, V. 1996, det. M., Marković, 4679, 4685 HMN); Dobre vode, UTM EN46 (leg. Jovanović, V. 1976, det. V., Jovanović, 5609 HMN); Ivanjske livade, UTM EN46, livada (leg. Jovanović, V. 1976, det. V., Jovanović, 5608 HMN); Petrova gora, UTM EN46, livade (leg. Nikolić, V. 21-Jul-1959, det. N., Diklić, 43410 BEO); 1400 m, bukova šuma (leg. Nikolić, v. 23-Jul-1959, det. N., Diklić, 43409 BEO); Sijarinjska Banja, UTM EN43, hrastovo-grabova šuma, kamenjari (leg. Nikolić, V. 23-Jun-1965, det. N., Diklić, 43414 BEO); Vranje: Ćoška, ul. Zelengorska, UTM EN71, 23-Jul-1999, BEOU-KEGB); SE Serbia Bosilegrad, UTM FN20 (leg. Ranđelović, N. 1990, det. N., Ranđelović, 125 HMN); Izvor, UTM FN20 (leg. Ranđelović, N. 2002, det. N., Ranđelović, 2701 HMN); Čemernik: Ostrozub, UTM FN04, 07-Aug-1950, 42936 BEOU); 13-Jul-1950, 42900 BEOU); Kozjak: Koviljski rit, Jamina greda, UTM EM78, 14-Jul-1961, 42897 BEOU); Trgovište: Radovica, UTM EN71, pored puteva (leg. Nikolić, V., Diklić, N., Bogdanović, M. 15-Jul-1972, det. V., Nikolić, 43524 BEO, sub. H. perforatum subsp. veronense, rev. B., Zlatković 01-Apr-2014); Vlasina, škriljci, pukotine stena (leg. Černjavski, $P$. 08-Aug-1930, det. P., Černjavski, 8662 BEO); 1200 m, livade (leg. Diklić, N. 23-Jul-1966, det. N., Diklić, 43493 BEO); pored puteva (leg. Dikliić, N. 16-Jul-1985, det. N., Diklić, 43415 BEO); (leg. Ranđelović, V. 1998, det. V., Ranđelović, $1318 \mathrm{HMN}$ ); Vranje: Aleksandrovac, UTM EP01, livada (leg. Zlatanović, S. 2006, det. B., Zlatković, 4204 HMN); Pržar, UTM EN71 (leg. Jovanović, V., Mišić, V., Dinić, A. 1983, det. M., Marković, 4614 HMN); Vranjska Banja, UTM EN81 (leg. Ničić, Đ. 1886, 42566 BEOU); Kosovo Čičavica: Gradica, UTM DN92, hrastova šuma (leg. Diklić, N., 24-Jul-1961, det. N., Diklić, 43425 BEO); Gnjilane, UTM EN30, hrastova šuma, 01-Jul-1985, det. V., Nikolić, 43495 BEO); Vilina kosa?, UTM EN40, hrastova šuma (leg. Nikolić, V. 01-Jul-1965, 43495 BEO); Kosovska Mitrovica, UTM DN84, ruderalne površine (leg. Milinčić, D. 20-Jul-1996, det. D., Milinčić, 196 BEOU-KEGB); Zvečan, UTM DN85 (leg. Soška, Th. 22-Jun-1914, 42588 BEOU); Lipovica, UTM DM67, hrastova šuma (leg. Diklić, N. 24-Jun-1962, det. N., Diklić, 43424 BEO); (leg. Nindić, V. 24-Jun-1902, 42948 BEOU); Šar-planina (leg. Stešević, D. 22-Jun-2000, det. D., Stešević, 11 BEOU-KEGB); Brezovica, UTM DM97, serpentinit (leg. Stevanović, V. 12-Aug-2003, 3880 BEOU-KEGB); Metohija Mokra planina: Vrelo, UTM DN65, obala potoka (leg. Rudski, I., 02-Jul-1932, det. M., Jovanović, 43466 BEO); 
Paštrik: Gorožup, UTM DM66, 500-1500 m, krečnjak, livade i šume, Quercetum macedonicae (leg. Dikliić, N. 27-Jun-1979, det. V., Nikolić, N., Diklić, S., Mladenović, 43412 BEO); Peć: Gubavac, UTM DN32 (leg. Stevanović, V. 07-Jul-1978, 2484 BEOU-KEGB); Prokletije: Dečani, UTM DN40, šume pitomog kestena (leg. Janković, M. 18-Jun-1957, 42974 BEOU); (leg. Janković, M. 22-May-1958, 42959 BEOU).

\section{Hypericum perforatum subsp. Iatifolium (Gaudin) A. Frohl. (Fig. 4)}

NW Serbia Jablanik, UTM CP99, 1274 m, livade (leg. Diklić, N., Nikolić, V., 24-Jul-1954, det. V., Nikolić, 43492 BEO, sub. H. perforatum, rev. B., Zlatković 01-Apr-2014); utrina (leg. Diklić, N., Nikolić, $v$. 27-Jul-1954, det. V., Nikolić, 43438 BEO, sub. H. perforatum, rev. B., Zlatković 01-Apr-2014); Nova Varoš: Akmačići, Uvac - kanjon, UTM DP00, 1000 m, krečnjak, kamenjar (leg. Diklić, N., Nikolić, V. 10-Jul-1977, det. N., Diklić, 43404 BEO, sub. H. perforatum, rev. B., Zlatković 01-Apr-2014); W Serbia Tara: Zaovine, UTM CP65, 1250 m (leg. Lindtner, V., 28-Jun-1956, det. M., Jovanović, 43467 BEO, sub. H. perforatum, rev. B., Zlatković 01-Apr-2014); E Serbia Suva planina: Trem, UTM EN98, 1500 m, krečnjak, kamenjar (leg. Diklić, N., Nikolić, V., Mladenović, S., 26-Jul-1977, det. N., Diklić, 43403 BEO, sub. H. perforatum, rev. B., Zlatković 01-Apr-2014); S Serbia Rujan, UTM EM68, EM69, andezit, brdski pašnjaci (leg. Gudžić, B., Ranđelović, V., 29-Jul-1998, det. N., Ranđelović, 43453 BEO, sub. H. perforatum, rev. B., Zlatković 01-Apr-2014); SE Serbia Besna Kobila, UTM FN00, 1600-1900 m, livade i pašnjaci (leg. Diklić, N., Nikolić, V., 17-Jul-1968, det. N., Diklić, 43442 BEO, sub. H. perforatum, rev. B., Zlatković 01-Apr-2014); Kosovo Gnjilane: Kmetovce - Dobrovčane, Česma Miloševo, UTM EN40 (leg. Nikolić, V., 26-Jun-1966, 43497 BEO, sub. H. perforatum, rev. B., Zlatković 01-Apr-2014); Šar-planina: Gine vode, UTM DM96 (leg. Janković, M. 08-Jul-1961, 42922 BEOU); Ostrovica, UTM DM97, silikat (leg. Janković, M. 15-Jul-1967, 42957, 42958 BEOU); Prevalac - Gornje Selo, UTM DM96, 1500 m (leg. Diklić, N., Nikolić, V. 24-Jul-1980, det. N., Diklić, 43401 BEO, sub. H. perforatum, rev. B., Zlatković 01-Apr-2014); Metohija Šar-planina: Kobilica, Drajčići, UTM DM86, livade i pašnjaci (leg. Diklić, N., Nikolić, V., Mladenović, S., 19-Jul-1977, det. N., Diklić, 43411 BEO, sub. H. perforatum, rev. B., Zlatković 01-Apr-2014).

\section{Hypericum perforatum subsp. veronense (Schrank) H. Lindb. (Fig. 4)}

Banat Deliblatska peščara: Devojački bunar, UTM DQ98 (leg. Stevanović, V., 24-Jul-2011, 37326 BEOU-KEGB); Flamunda, UTM EQ07 (leg. Soška, Th. 22-Jul-1943, det. Th., Soška, 8645 BEO); Šumadija Kragujevac: Dobrača, Sjeničko polje, UTM DP78 (leg. Rudski, I., 24-Apr-1938, 8638 BEO); Šumarice, UTM DP84 (leg. Rudski, I. 30-Jul-1939, 8637 BEO); NE Serbia Kladovo, UTM FQ24 (leg. Pančić, J., det. J., Pančić, 3351 BEOU (Herb. Panc.), sub H. arenicola, rev. B., Zlatković 06-Feb-2014); C Serbia Ibar - dolina: Dobre strane, UTM DP62, livada (leg. Nikolić, V., Diklić, N., 12-Jul-1967, det. N., Diklić, 43502 BEO, sub. H. perforatum, rev. B., Zlatković 01-Apr-2014); Kruševac: Gaglovo, UTM EP32, pored puteva (leg. Bogdanović, M. 13-Aug-1971, det. V., Nikolić, 43521 BEO); Novi Pazar: Kladnica, UTM DP20, stene (leg. Nikolić, V., Mladenović, S. 17-Jul-1981, det. N., Diklić, 43320 BEO, sub H. linarioides, rev. B., Zlatković 31-Mar-2014); E Serbia Jerma klisura: Zvonačka Banja, UTM FN25 (leg. Nikolić, V., Diklić, N., 20-Jul-1965, det. N., Diklić, 43443 BEO, sub. H. perforatum, rev. B., Zlatković 01-Apr-2014); Stara planina: Midžor, UTM FP30, 1600 m, livada (leg. Lindtner, V. 20-Aug-1955, det. M., Jovanović, 43531 BEO); Udica - dolina, UTM FP30, 1924 m (leg. Nikolić, V., Diklić, N. 23-Jun-1958, 43413 BEO, sub. H. perforatum, rev. B., Zlatković 01-Apr2014); Vidlič: Paterica?, UTM FN48, kamenjari, livade (leg. Marković, M. 29-Jul-2009, det. M., Marković, $9051 \mathrm{HMN}$ ); SW Serbia Priboj: Bistrica, UTM CP91, serpentinit, kamenjar (leg. Nikolić, V., Diklić, N., 11-Jul-1977, det. N., Diklić, 43318, 43319 BEO, sub H. linarioides, rev. B., Zlatković 31Mar-2014); Crni vrh, UTM CP82 (leg. Lindtner, v. 22-Jul-1949, 8630 BEO, sub. H. perforatum, rev. B., Zlatković 01-Apr-2014); Prijepolje: Strugovi, UTM CN99, 30-Jul-1930, 42607 BEOU); S Serbia Bujanovac: Končuljska klisura, UTM EN50 (leg. Nikolić, V., Diklić, N., Bogdanović, M., 18-Jul-1974, det. V., Nikolić, 43523 BEO); SE Serbia Vlasina: Brana, UTM FN03, vlažne livade (leg. Nikolić, V., Diklić, N., Bogdanović, M. 25-Jul-1972, det. V., Nikolić, 43525 BEO); Vranjska Banja, UTM EN81 (leg. Ničić, Đ. 27-Jul-1886, 43083 BEOU); Kosovo Šar-planina: Careve livade, UTM DM96, šuma munike i belog bora (leg. Janković, M., 22-May-1958, 42934 BEOU). 


\section{Hypericum tetrapterum Fries (Fig. 5)}

Banat Deliblatska peščara: Majur Bara, UTM EQ16, šumarci (leg. Stevanović, V., 14-Jul2011, 37329 BEOU-KEGB); Šumadija Kragujevac: Bubanj, Barebanj?,UTMDP97 (leg. Pančić, J., det. J., Pančić, 3428 BEOU (Herb. Panc.)); Topola: Vlakča, UTM DP78 (leg. Pančić, J., det. J., Pančić, 3427 BEOU (Herb. Panc.)); Pomoravlje Smederevska Palanka, UTM DQ91, zamočvarena mesta, obala potoka (leg. Pečnik, 28-Aug-1943, det. P., Černjavski, $8692 \mathrm{BEO}$ ); Kusadak, UTM DQ81, pored puteva (leg. Tomić, A. 14-Jul-1992, det. S., Jovanović, 42804 BEOU, sub H. acutum); NE Serbia Đerdap: Tekija, UTM FQ14 (leg. Pančić, J., det. J., Pančić, 3430 BEOU (Herb. Panc.)); Majdanpek: Debeli Lug, Pek - dolina, UTM EQ71 (leg. Černjavski, P. 08-Aug-1946, det. P., Černjavski, 8628 BEO, sub H. perforatum, rev. B., Zlatković 01-Apr2014); Zlot: Podgorac, UTM EP76 (leg. Dimitrijević, M., S. 01-Jul-1992, 1367 BEOUKEGB); W Serbia Klisura Panjice, UTM DP23, kamenjari, stene i sipari (leg. Lakušić, D., Jovanovć, S., 28-Jul-1990, 2202 BEOU-KEGB, sub H. acutum, rev. B., Zlatković 12-Dec-2013); (leg. Stanić, S., Bemić, N. 01-Aug-1990, 2737 BEOU-KEGB, sub H. acutum, rev. B., Zlatković 12-Dec-2013); Nova Varoš: Uvac - ka-

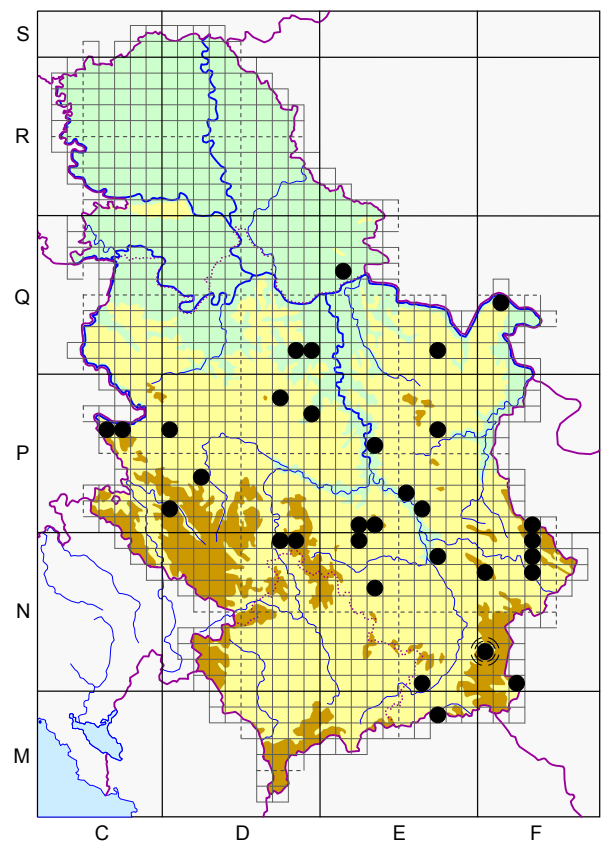

Fig. 5. - Distribution of the species $H$. tetrapterum Fries in Serbia. njon, UTM DP01, Exp. SW, 900 m, krečnjak, šikare, Fraxinus ornus i Crategus monogyna (leg. Lakušić, D. 23-May-1996, 300 BEOU-KEGB, sub H. maculatum, rev. B., Zlatković 06-Feb-2014); Tara: Batura - Kruščica, UTM CP66, vlažne livade (leg. Stevanović, V., Vukojičić, S. 30-Aug-2000, 14456 BEOUKEGB); Mitrovac - Krnja jela, UTM CP76, zatresavljna livada (leg. Stevanović, V., Jovanović, S., Vukojičić, S. 13-Aug-2000, 14305 BEOU-KEGB); Užice: Jelova gora, UTM DP06 (leg. Lakušić, D., Vukojičić, S. 25-Jul-1999, 9250 BEOU-KEGB, sub H. maculatum, rev. B., Zlatković 06-Feb-2014); $\underline{\mathbf{C}}$ Serbia Blace, UTM EN29 (leg. Pančić, J., det. J., Pančić, 3432 BEOU (Herb. Panc.)); Kopaonik: Semeteško jezero, UTM DN79 (leg. Pančić, J., det. J., Pančić, 3431 BEOU (Herb. Panc.)); Suvo rudište, prema mauzoleju, UTM DN89 (leg. Nikolić, V., Diklić, N. 18-Sep-1964, det. N., Diklić, 43533 BEO, sub H. acutum, rev. B., Zlatković 01-Apr-2014); Veliki Jastrebac, UTM EP20, EP30 (leg. Pančić, J., det. J., Pančić, 3429 BEOU (Herb. Panc.)); E Serbia Aleksinac, UTM EP52 (leg. Perić, 1929, 42805 BEOU); Vrelo, UTM EP61 (leg. Niketić, M., Tomović, G. 16-Sep-1998, 11240 BEOU-KEGB, sub H. maculatum, rev. B., Zlatković 06-Feb-2014); Bela Palanka: Šljivovički vrh, Gornja Koritnica, UTM FN07, 535 m, krečnjak, obala potoka (leg. Jušković, M. 15-Jul-2004, 1005 BEOU-KEGB); Koritnička reka - klisura, UTM FN07, 350 m, krečnjak, obala reke (leg. Jušković, M., Ranđelović, V. 23-Aug2003, 1004 BEOU-KEGB); Paraćin: Grza - klisura, UTM EP35, krečnjak, vlažne livade (leg. Pejanović, V. 13-Aug-2000, 203 BEOU-KEGB, sub H. acutum, rev. B., Zlatković 06-Feb-2014); Pirot: Visočica - klisura, UTM FN39 (leg. Cvetković, D. 20-Jul-1991, 2669 BEOU-KEGB, sub H. tertium, rev. B., Zlatković 12-Dec-2013); Seličevica: Donje Vlase, UTM EN78, 450 m, silikat, obala potoka, Fagetum moesiacae (leg. Zlatković, B. 1998, det. B., Zlatković, 7266 HMN); Stara planina: Babin zub, UTM FP30, planinske livade (leg. Marković, M. 2005, det. V., Ranđelović, 1399 HMN); Suva planina: Gornja Koritnica, UTM FN07, 535 m, krečnjak, obala potoka, 1005 BEOU); Koritnička reka klisura, UTM FN07, 350 m, krečnjak, obala reke, 1004 BEOU); Vidlič: Basarski kamik, UTM FN37, obala potoka (leg. Marković, M. 12-Jul-2008, det. D., Pavlović, 4866 HMN, sub H. acutum); Crni vrh, Grlja, UTM FN38, livada (leg. Marković, M. 6.2004, det. M., Marković, 1122 HMN, sub H. acutum); Vazganica, UTM FN38, opožarena mesta, bukove šume (leg. Marković, M. 07-Jul-2010, det. M., Marković, 10299 HMN, sub H. acutum); S Serbia Bujanovac: Lopardince, UTM EN60, tresave i 
Nardetum strictae (leg. Diklić, N., 23-Sep-1961, det. N., Diklić, 43478 BEO, sub H. perforatum subsp. vulgare, rev. B., Zlatković 01-Apr-2014); Radan: Velika Loparda, UTM EN36, tresava (leg. Jovanović, V. 1976, det. M., Marković, $4921 \mathrm{HMN}$ ); (leg. Jovanović, V. 1976, det. V., Jovanović, 5600 HMN); SE Serbia Kozjak: Brestov Vrtlog?, UTM EM78, 18-May-1961, 42909 BEOU); Koviljski rit, Široka mlaka, UTM EM78, 18-May-1961, 42898 BEOU); Rudina, UTM FN20 (leg. Ranđelović, N. 2003, det. N., Ranđelović, 2375 HMN, sub H. acutum); (leg. Ranđelović, N. 2004, det. N., Ranđelović, $2582 \mathrm{HMN}$, sub H. acutum); Vlasina, UTM FN02, pored puteva (leg. Nikolić, V. 18-Jul1958, det. N., Diklić, 43532 BEO, sub H. acutum, rev. B., Zlatković 01-Apr-2014).

\section{H. sect. OLYMPIA (Spach) Nyman}

\section{Hypericum olympicum L. (Fig. 6)}

S Serbia Rujan, UTM EM68, EM69, ass. Hyperico-Trifolietum trichopteri (leg. Ranđelović, N., Stamenković, V., 08-Jul-1997, det. V., Stamenković, 43376 BEO); Blizanci, UTM EM68, 900-1000 m, silikat (leg. Niketić, M., Tomović, G. 14-Jul-2006, 21370 BEOU-KEGB); Spančevac, UTM EM79, granit (leg. Ranđelović, N. 04-Jul-1983, det. N., Ranđelović, 43379 BEO); Svinjište, UTM EM68, $780 \mathrm{~m}$, silikat, suvi pašnjaci (leg. Zlatković, B., Tomović, G. 2013, det. B., Zlatković, 7249 HMN); SE Serbia Pčinja - dolina: manastir Sv. Prohor Pčinjski, UTM EM78 (leg. Tomović, G., Ranđelović, V., Zlatković, B., 08-Aug-1997, 8141 BEOU-KEGB).

\section{H. sect. DROSOCARPIUM Spach}

\section{Hypericum barbatum Jacq. (Fig. 7)}

NW Serbia Maljen, UTM DP28, serpentinit, kamenjari, zona bukve (leg. Diklić, N., Bogdanović, M., 03-Jul-1984, 43204 BEO); Crni vrh, UTM DP18, šuma, 13-Jul-1949, det. N., Diklić, 43206 BEO); 1200 m, serpentinit, det. M., Jovanović, 43196 BEO); Divčibare, UTM DP18, 950 m, 1948, 8804 BEO); 27-Jul-1948,

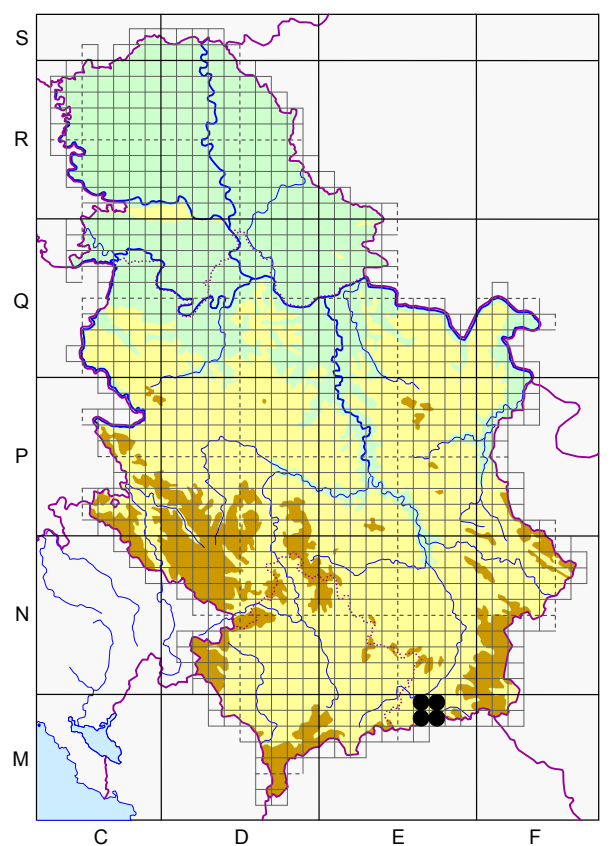

Fig. 6. - Distribution of the species H. olympicum L. in Serbia. det. N., Diklić, 43205 BEO); 950 m, serpentinit, livada (leg. Diklić, N., Mladenović, S. 02-Jul-1984, det. M., Jovanović, 43197 BEO); serpentinit (leg. Stevanović, v. 09-Sep-1981, 2682 BEOU-KEGB); Tometino polje, UTM DP18, 800 m, serpentinit, kamenjar (leg. Jovanović, S., Lakušić, D., Vukojičić, S. 21-Aug-2009, 29738 BEOU-KEGB); Šumadija Aranđelovac: Venčac, Brajinac, UTM DQ60, serpentinit, kamenjar (leg. Niketić, M., Tomović, G., 21-May-2005, 19735 BEOU-KEGB); Avala, UTM DQ64, 07-Jun1952, 42918 BEOU); (leg. Pančić, J., det. J., Pančić, 3356 BEOU (Herb. Panc.)); Borač: Borački krš, UTM DP66, gabro, stene (leg. Stevanović, V. 08-Jun-2003, 22309 BEOU-KEGB); Gornji Milanovac: Brđanska klisura, UTM DP57, serpentinit (leg. Stevanović, V. 27-Jul-1983, 23130 BEOU-KEGB); Kragujevac: Borački krš, UTM DP66 (leg. Rudski, I. 07-Jul-1937, det. I., Rudski, 8794 BEO); Šumarice, UTM DP84 (leg. Rudski, I. 06-Jun-1936, det. I., Rudski, 8797 BEO); (leg. Rudski, I. 21-May1940, det. I., Rudski, 8799 BEO); (leg. Rudski, I. 30-May-1936, det. I., Rudski, 8800 BEO); Rudnik, UTM DP68, livade (leg. Diklić, N. 10-Jun-1963, det. N., Diklić, 43209 BEO); mešovita listopadnočetinarska šuma (leg. Jovanović, M. 10-Jun-2003, det. M., Jovanović, 43228 BEO); Pomoravlje Jagodina: Đurđevo brdo, UTM EP26 (leg. Pančić, J., det. J., Pančić, 3352 BEOU (Herb. Panc.)); 


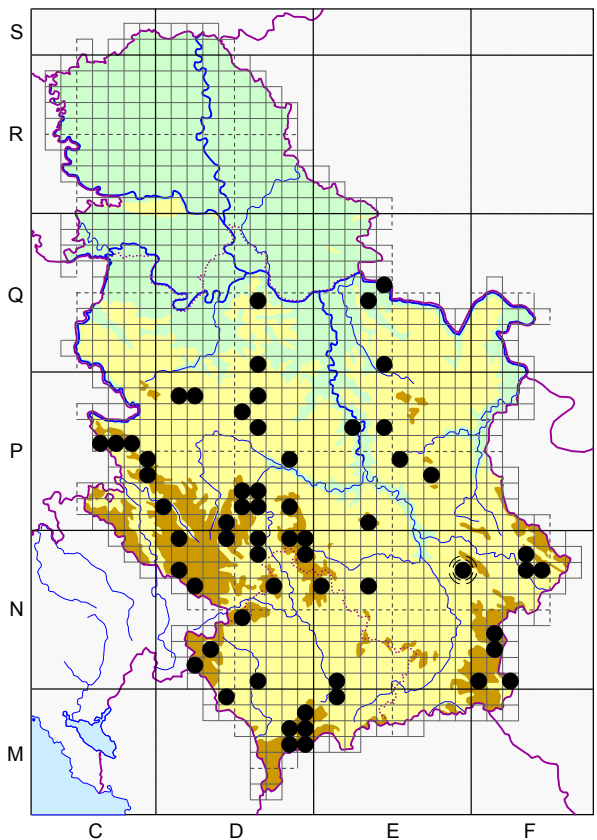

Fig. 7. - Distribution of the species

H. barbatum Jacq. in Serbia.
Paraćin: manastir Ravanica, UTM EP46 (leg. Pančić, J., det. J., Pančić, 3353 BEOU (Herb. Panc.)); Požarevac: Ljubinje, UTM EQ34, 42647 BEOU); NE Serbia Đerdap: Vinci, UTM EQ45 (leg. Pančić, J., det. J., Pančić, 3360 BEOU (Herb. Panc.)); Homolje: Vukan, UTM EQ40 (leg. Pančić, J., det. J., Pančić, 3359 BEOU (Herb. Panc.)); W Serbia Mokra Gora: Šargan, UTM CP85, serpentinit, kamenjar (leg. Stevanović, V., 2002, BEOU-KEGB); Tara: Miloševac, UTM CP75 (leg. Pančić, J., det. J., Pančić, 3354 BEOU (Herb. Panc.)); Mitrovac - Zaovine, UTM CP65, serpentinit, kamenjar (leg. Stevanović, V. 05-Aug-2001, 12494 BEOUKEGB); Zlatibor, livade (leg. Bogdanović, $M$. 13-Aug-1974, det. V., Nikolić, 43240 BEO); (leg. Jurišić, Ž. 23-Jun-1912, 8802 BEO); UTM CP94, 900-1000 m, serpentinit, livade, kamenjari (leg. Jovanović, M., Niketić, M. 22-Jun2004, det. M., Jovanović, 43223, 43226 BEO); Borova Glava, UTM CP85, 27-Jun-2004, 16054 BEOU-KEGB); Gmizova ćuprija, UTM CP93 (leg. Jurišić, Ž. 15-Jun-1912, 8801 BEO); Partizanske vode, UTM CP94, 90 m, serpentinit, livade (leg. Diklić, N., Mladenović, S. 04-Jul-1974, det. M., Jovanović, 43234 BEO); Ribnica - jezero, UTM CP93, $900 \mathrm{~m}$, šuma belog bora (leg. Diklić, N., Mladenović, S. 05-Jul1984, det. M., Jovanović, 43241 BEO); C Serbia Ibar - dolina: Dobre strane, UTM DP62, serpentinit (leg. Diklić, N., 27-May-1971, det. N., Diklić, 43233 BEO); livada (leg. Nikolić, V., Diklić, N. 12Jul-1967, det. N., Diklić, 43203 BEO); Maglić, UTM DP62, serpentinit (leg. Lakušić, D. 30-Jun-1998, 8728 BEOU-KEGB); Stolovi, Greda, UTM DP52, 1150-1320 m, livade (leg. Nikolić, V., Diklić, N. 12-Jul1967, det. N., Diklić, 43232 BEO); Ušće, UTM DP61 (leg. Rudski, I. 1940, det. I., Rudski, 8798 BEO); Kopaonik: Golo Brdo, UTM DN89, serpentinit, 12-Aug-1986, 42530 BEOU); Karanovac, UTM CP93 (leg. Pančić, J., det. J., Pančić, 3355 BEOU (Herb. Panc.)); Kozje stene, UTM DN99, BEOUKEGB); 1400 m, krečnjak, kamenjar, Potentilleto- Caricetum humilis (leg. Lakušić, D. 17-Jul-1987, det. D., Lakušić, BEOU-KEGB); 1400 m, krečnjak, kamenjar, Seslerietum rigidae (leg. Lakušić, D., Niketić, M. 10-Aug-1986, det. D., Lakušić, BEOU-KEGB); Oštro koplje, UTM EN06 (leg. Soška, Th. 29-Jun1814, BEOU); Treska, UTM DN98, 1500 m, livade i pašnjaci, Agrostidetum capillare (leg. Lakušić, D., Niketić, M. 07-Aug-1986, det. D., Lakušić, BEOU-KEGB); serpentinit (leg. Niketić, M., Tomović, G., Novčić, R. 09-Jul-2005, 19927 BEOU-KEGB); serpentinit, stene, rudine (leg. Niketić, M., Tomović, G., Novčić, R. 26-Jun-2004, 18955 BEOU-KEGB); (leg. Rudski, I. 14-Jul-1938, det. I., Rudski, 8795 BEO); Kraljevo: Čukojevac, UTM DP84 (leg. Rudski, I. 16-Jun-1940, det. I., Rudski, 8796 BEO); Radočelo, UTM DP51 (leg. Soška, Th. 02-Jul-1932, 42643 BEOU); Raška, UTM DN69, serpentinit, kamenjar (leg. Stevanović, V., Vukojičić, S., Tomović, G. 15-Jun-2003, 16924 BEOU-KEGB); Sokolovica: Mali krš, UTM EN36, 930 m, andezit, stene, 1456 BEOU); (leg. Tomović, G., Tomović, M. 24-Jul-1998, 1456 BEOUKEGB); Stolovi, UTM DP62, serpentinit (leg. Jovanović, M., Niketić, M. 21-Jun-2004, det. M., Jovanović, 43227 BEO); (leg. Košanin, N. 22.08., 42624 BEOU); Veliki Jastrebac: Pogled, UTM EP30, 1400 m, pašnjaci, kamenjari (leg. Diklić, N. 25-Jul-1959, det. N., Diklić, 43210 BEO); Željin, UTM DP81, serpentinit (leg. Stevanović, V. 23-Jun-1989, 4188 BEOU-KEGB); (leg. Stevanović, V., Jovanović, S. 23Jun-1989, 4215 BEOU-KEGB); Baćište, UTM DP81, 21-Jun-1989, 45205 BEOU); Brezova, UTM DP51, ass. Koelerio-Danthonietum alpinae (leg. Stevanović, v. 23-Jun-1989, 4010 BEOU-KEGB); E Serbia Rtanj: Šarbanovac, UTM EP73, 675 m, krečnjak, termofilni pašnjaci (leg. Đorđević, A., 2010, det. B., Zlatković, 7259 HMN); Vrelski potok, UTM EP54 (leg. Rudski, I. 02-Jul-1932, det. M., Jovanović, 43242 BEO); Suva planina, UTM EN97, 06-Jul-1911, 43086 BEOU); (leg. Dimitrijević, M. M. 
06-Jul-1911, det. Ž., Jurišić, 8792 BEO); Vidlič, UTM FN47, kamenjar (leg. Marković, M. 11-Jun-2005, det. M., Marković, 1974 HMN); Basarski kamik, UTM FN38, opožarena mesta, bukove šume (leg. Marković, M., Dimitrijević, M., D., Marković, Lj. 27-Sep-2009, det. M., Marković, 8034 HMN); Gulenovci, UTM FN47, kamenjar (leg. Marković, M. 11-Jun-2005, det. M., Marković, 146 HMN); Krupac, UTM FN37, šuma (leg. Marković, M., Marković, Lj., Stojanović, Lj., Đorđević, L. 14-Jun-2008, det. M., Marković, $4340 \mathrm{HMN}$ ); SW Serbia Golija, UTM DN49, livade i proplanci, pojas smrče (leg. Nikolić, V., Mladenović, S., 09-Jul-1982, det. M., Jovanović, 43198 BEO); Dajići, UTM DP40, pored tresave (leg. Kalafatić, V. 24-Jun-1994, 4432 BEOU-KEGB); Studenica, UTM DP61, kamenjar (leg. Stevanović, V., Vukojičić, S., Tomović, G. 14-Jun-2003, 16829 BEOU-KEGB); Pešter, pašnjaci, livade (leg. Nikolić, V., Diklić, N., Mladenović, S. 19-Jul-1980, det. M., Jovanović, 43199 BEO); Giljeva, UTM DN17, 1300 m, krečnjak, pašnjaci, kamenjari (leg. Jovanović, S. 23-Jun-1996, 2640 BEOU-KEGB); Suvi Do (Suhodol)?, UTM DN26 (leg. Košanin, N., 43080 BEOU); Raška: Merćez, UTM DN68, livade (leg. Košanin, N. 21-Jun-1926, 43081 BEOU); Rogozna: Čapljinac, UTM DN76, 1318 m, serpentinit, pašnjaci, kamenjari (leg. Niketić, M., Tomović, G. 08-Jul-2010, 30720 BEOU-KEGB); Grižani Han, UTM DN76 (leg. Soška, Th. 24-Jun-1914, 42032 BEOU); Velika Skakavica, UTM DN76, 1166 m, serpentinit, pašnjaci, Carex humilis i Bromus erectus (leg. Niketić, M., Tomović, G. 08-Jul-2010, 30732 BEOU-KEGB); Sjenica, UTM DN19, serpentinit, livade, kamenjari (leg. Jovanović, S. 23-Jun-1996, 2724 BEOU-KEGB); (leg. Jovanović, S. 23-Jun-1996, 2742 BEOU-KEGB); Zlatar: Zlatarsko jezero, UTM DP01, 872 m, serpentinit (leg. Kabaš, E., Kuzmanović, N., Vukojičić, S. 21-Jun-2012, 34700 BEOUKEGB, sub H. rumeliacum, rev. B., Zlatković 06-Feb-2014); SE Serbia Besna Kobila, UTM FN00 (leg. Jovanović, V., 1976, det. V., Jovanović, 4418 HMN); 1600-1900 m, livade i pašnjaci (leg. Nikolić, V., Diklić, N. 17-Aug-1968, det. N., Diklić, 43230 BEO); 1800 m, livade (leg. Nikolić, V., Diklić, N. 22-Jul-1967, det. N., Diklić, 43200 BEO); 1900 m, pašnjaci, kamenjari (leg. Nikolić, V., Diklić, N. 22-Jul-1967, det. N., Diklić, 43201 BEO); 1650-1900 m, pašnjaci, kamenjari (leg. Diklić, N., Mladenović, S. 15-Jul-1979, det. N., Diklić, 43635 BEO, sub H. rumeliacum, rev. B., Zlatković 31-Mar-2014); planinarski dom, UTM FN00, 1500-1600 m, livade (leg. Nikolić, V., Diklić, N. 22-Jul-1967, det. N., Diklić, 43202 BEO); Rudina, UTM FN20 (leg. Ranđelović, V. 1992, det. V., Ranđelović, 2011 HMN); Vlasina: Mala Plana, UTM FN13 (leg. Pančić, J., det. J., Pančić, 3357 BEOU (Herb. Panc.)); Stratorija, UTM FN12, 10.07., 42644 BEOU); (leg. Košanin, N. 8.191, 42629 BEOU); Kosovo Kodža Balkan: Golem bor, UTM DM87, šuma molike (leg. Janković, M., 11-Jul-1961, 42941 BEOU); (leg. Janković, M. 25-Apr-1965, 42927 BEOU); Jezerska planina, UTM DM98, 1600-2090 m, serpentinit, pašnjaci, kamenjari (leg. Nikolić, V., Diklić, N. 18-Jul-1975, det. V., Nikolić, 43238 BEO); Kopaonik: Oštro koplje, UTM EN06 (leg. Soška, Th. 29-Jun-1914, 42645 BEOU); Koprivnik, UTM DN32, 1600 m (leg. Grebenščikov, O. 13-Jul1939, det. O., Grebenščikov, 8808 BEO); Priština: Lipljane, UTM EN10, 500 m, šuma sladuna i cera (leg. Diklić, N. 29-May-1971, det. N., Diklić, 43207 BEO); Šar-planina (leg. Stevanović, V., Jovanović, S. 21-Jul-1989, 3345 BEOU-KEGB); Brezovica, Malo Borče, UTM DM97, 1400-1512 m (leg. Nikolić, V., Diklić, N., Bogdanović, M. 21-Jul-1974, det. V., Nikolić, 43239 BEO); Gine vode, UTM DM96 (leg. Janković, M. 07-Jul-1971, 42953 BEOU, sub H. montanum, rev. B., Zlatković 12-Dec-2013); Ošljak, UTM DM97, 1800-2000 m (leg. Rudski, I. 23-Jul-1930, 42649 BEOU); 2000 m, krečnjak (leg. Nikolić, V., Diklić, N., Mladenović, S. 23-Jul-1976, det. N., Diklić, 43557 BEO, sub H. richeri subsp. grisebachii, rev. B., Zlatković 31-Mar-2014); 1800-2212 m, krečnjak, kamenjar (leg. Nikolić, V., Diklić, N., Bogdanović, M. 19-Jul-1974, det. V., Nikolić, 43591 BEO, sub H. umbellatum, rev. B., Zlatković 31-Mar-2014); Cerevački vrh, UTM DM97, 1500-2040 m, krečnjak (leg. Nikolić, V., Diklić, N. 19-Jul-1975, det. V., Nikolić, 43237 BEO); Virovi - Golem bor, UTM DM87, 1900 m (leg. Rudski, I. 22-Jul-1930, 42648 BEOU); (leg. Rudski, I. 23-Jul-1930, 42650 BEOU); Uroševac: Sazlija, UTM EM19, livade (leg. Jurišić, Ž. 04-May-1914, 8810 BEO); Metohija Koznik: Miruša, UTM DN60, 370-500 m, serpentinit (leg. Diklić, N., 28-May-1971, det. N., Diklić, 43208 BEO); Mokra gora: Slomna gora, UTM DN54, opožarena šumska staništa (leg. Rudski, I. 06-Jul-1932, det. M., Jovanović, 43243 BEO); Planik: Ponoševac, UTM DM49, serpentinit (leg. Košanin, N. 20-May-1913, 43040 BEOU); Prizren: Drven Grad, UTM DM87 (leg. Rudski, I. 25-Jul-1930, 42651 BEOU); Prokletije: Pločica, UTM DN21, šuma molike (leg. Černjavski, P., Rudski, I., Lindtner, V. 18-Jul-1933, det. P., Černjavski, 8791 BEO; Šarplanina: Kobilica, UTM DM86, 1650 m (leg. Rudski, I. 04-Jul-1930, 42646 BEOU). 


\section{Hypericum montbretii Spach (Fig. 8)}

S Serbia Preševo: Kozjak, Delinovački rid, UTM EM78, migmatiti, 16255 BEOU); Golemi uši, UTM EM78, migmatiti, BEOU); Pčinja dolina, Vogance, UTM EM78, biotit i gnajs, 16258 BEOU); Starac, Gornji Starac, UTM EM78, biotit i gnajs, 16260 BEOU); karaula, UTM EM79, biotit i gnajs, 16256 BEOU); SE Serbia Kozjak: Delinovički rid, UTM EM78, $640 \mathrm{~m}$, migmatiti, polidominantne hrastove šume, Carpino orientalis-Quercetum mixtum, BEOU); Pčinja - dolina: Kostin čukar, UTM EM79, $800 \mathrm{~m}$, biotit i gnajs, šume kitnjaka, Quercetum montanum, BEOU); Vogance, UTM EM78, 440 $\mathrm{m}$, biotit i gnajs, šikare grabića i medunca, Ostryo-Carpinion aegeicum, BEOU); Vogance Jablanica, Kostin čukar, UTM EM79, biotit i gnajs, 16259 BEOU); Starac, UTM EM78, 590 $\mathrm{m}$, migmatiti, šikare grabića i medunca, OstryoCarpinion aegeicum, BEOU); Gornji Starac, UTM EM78, $800 \mathrm{~m}$, migmatiti, šikare grabića i medunca, Ostryo-Carpinion aegeicum, BEOU); Široka planina: Dumbije, UTM EM88, 650 $\mathrm{m}$, mikašisti i leptinoliti, šume kitnjaka, Quercetum montanum, BEOU); Trgovište: Široka planina, Dumbije, UTM EM88, mikašisti i leptinoliti, $16257 \mathrm{BEOU})$.

\section{Hypericum richeri subsp.} grisebachii (Boiss.) Nyman (Fig. 9)

NW Serbia Maljen, UTM DP28 (leg. Pančić, J., 43091 BEOU); (13-Jul-1949, det. N., Diklić, 43550 BEO, sub $H$. alpinum, rev. B., Zlatković 31-Mar-2014); NE Serbia Bor: Stol, UTM EP99 (leg. Nikolić, V., 23-Jun-1955, det. M., Jovanović, 43539 BEO); kamenjar (leg. Nikolić, V. 25-Jun-1955, det. M., Jovanović, 43538, 43540 BEO); W Serbia Perućac: Derventa, UTM CP66, stene, 08-May-1909, det. N., Pak, 8769 BEO, sub H. alpinum, rev. B., Zlatković 31-Mar2014); C Serbia Goč: Velika livada, UTM DP72, $1110 \mathrm{~m}$, livade, 26-Jul-1950, det. N., Diklić, 43551 BEO, sub H. alpinum, rev. B., Zlatković 31-Mar-2014); Kopaonik, 43089 BEOU); šuma smrče (leg. Diklić, N., Mladenović, S. 16-Jul1985, 43537 BEO); (leg. Pančić, J., det. J., Pančić, 3400, 3405 BEOU (Herb. Panc.)); (15-Jul-1987, BEOU-KEGB); (leg. Pančić, J., det. J., Pančić, 3339, 3340 BEOU (Herb. Panc.), sub H. alpinum, rev. B., Zlatković 06-Feb-2014); (leg. Ilić, K. 1919, 8618 BEO, sub H. humifusum, rev. B., Zlatković 31Mar-2014); Brzeće, UTM DN99, 29-Jun-2004, 16053 BEOU-KEGB); Gobelja, UTM DN89, $1800 \mathrm{~m}$, silikat, ass. Juniperetum nanae (leg. Sigunov, A. 20-Jul-1978, 43536 BEO, sub H. transsilva-

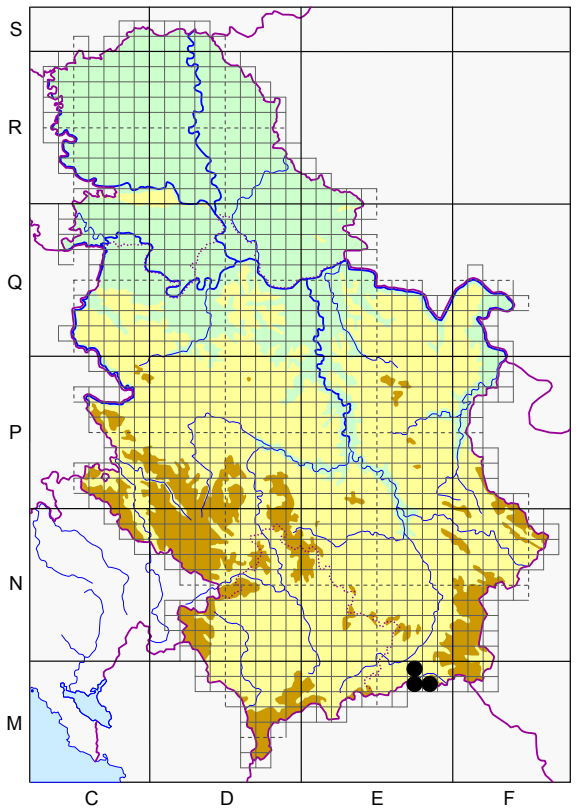

Fig. 8. - Distribution of the species H. montbretii Spach in Serbia.

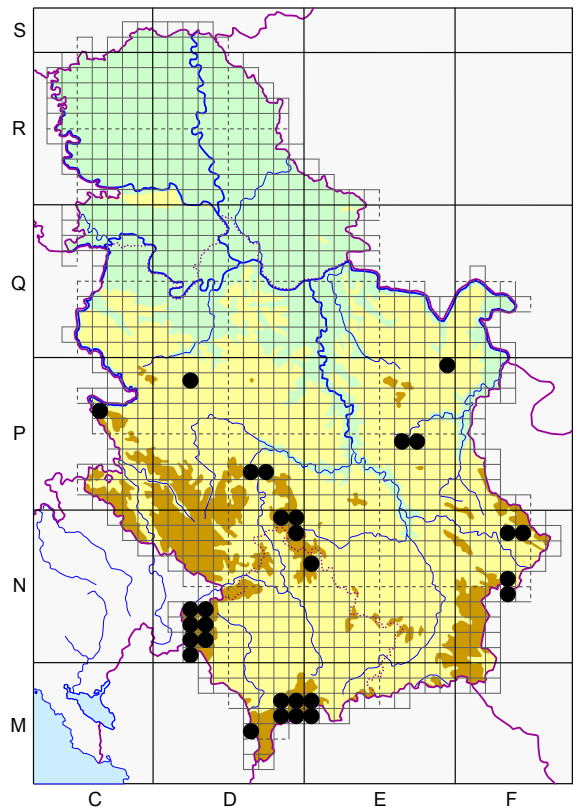

Fig. 9. - Distribution of the subspecies H. richeri subsp. grisebachii (Boiss.) Nyman in Serbia. 
nicum, rev. B., Zlatković 31-Mar-2014); Karaman, UTM DN89 (leg. Rudski, I. 09-Jul-1938, 8770 BEO, sub H. alpinum, rev. B., Zlatković 31-Mar-2014); Milanov vrh, UTM DN89 (leg. Rudski, I. 08-Jul1938, 8777 BEO, sub H. alpinum, rev. B., Zlatković 31-Mar-2014); (leg. Rudski, I. 13-Aug-1938, 8767 BEO, sub H. alpinum, rev. B., Zlatković 31-Mar-2014); Sedlo, UTM DN89, 1600 m, ass. VaccinioJuniperetum sibiricae, 12-Jul-1987, BEOU-KEGB); Suvo rudište, UTM DN89, livade (leg. Dimitrijević, M. M. 19-Jul-1911, det. Ž., Jurišić, 43571 BEO); (leg. Košanin, N. 17-Jul-1910, 42703 BEOU, sub H. alpigenum, rev. B., Zlatković 12-Dec-2013); 1900 m, kornit, ass. Poetum violaceae (leg. Stanić, S. 11Jul-1987, det. D., Lakušić, BEOU-KEGB, sub H. transsilvanicum, rev. B., Zlatković 06-Feb-2014); Treska, UTM DN98, serpentinit, stene, rudine (leg. Niketić, M., Tomović, G., Novčić, R. 26-Jun-2004, 18954 BEOU-KEGB); Stolovi, UTM DP62 (leg. Pančić, J., det. J., Pančić, 3338, 42705 BEOU BEOU (Herb. Panc.), sub H. alpestre, rev. B., Zlatković 06-Feb-2014); (leg. Pančić, J., det. J., Pančić, 3342 BEOU (Herb. Panc.), sub H. alpinum, rev. B., Zlatković 06-Feb-2014); E Serbia Greben, UTM FN34, FN35, pašnjaci (leg. Lindtner,V., 24-Jul-1933, det. P., Černjavski, 8771 BEO, sub H. alpinum, rev. B., Zlatković 31-Mar-2014); Rtanj, UTM EP64, EP74 (leg. Pančić, J., det. J., Pančić, 3408 BEOU (Herb. Panc.)); Vidlič: Krajište, UTM FN48, opožarena mesta, bukove šume (leg. Marković, M. 13-Jun-2010, det. M., Marković, 10144, $10183 \mathrm{HMN}$ ); Visoka stena, UTM FN38, opožarena mesta, bukove šume (leg. Marković, M., Stanković, M., Marković, Lj. 27-Jun-2009, det. M., Marković, 8697, 8703, 8725 HMN); Vazganica, UTM FN38, opožarena mesta, bukove šume (leg. Marković, M. 22-Jun-2008, det. D., Pavlović, 5480 HMN, sub H. alpinum subsp. grisebachii); Kosovo Kodža Balkan: Golem bor, UTM DM87, 1900 m, krečnjak, kamenjar (leg. Nikolić, V., Diklić, N., 19-Jul-1975, det. V., Nikolić, 43546 BEO, sub H. alpinum, rev. B., Zlatković 31-Mar-2014); Kopaonik: Oštro koplje, UTM EN06 (leg. Soška, Th., 42701 BEOU); Šar-planina: Brezovica, UTM EM06, kamenjar (leg. Nikolić, V., Diklić, N., Bogdanović, M. 22-Jul-1974, det. V., Nikolić, 43549 BEO, sub H. alpinum, rev. B., Zlatković 31-Mar2014); Careve livade, UTM DM96, 16-Jul-1930, 42708 BEOU); lovačka kuća, UTM DM96, 2000 m, u pojasu Pinus peuce (leg. Stevanović, v. 26-Jul-1978, 1676 BEOU-KEGB, sub H. alpigenum, rev. B., Zlatković 12-Dec-2013); Durlov potok - dolina, UTM EM06 (leg. Saboljević, M. 29-Jun-1997, 7417 BEOU-KEGB, sub H. alpigenum); Gine vode, UTM DM96, žbunjaci, Rhododendron (leg. Janković, M. 07-Jul-1971, 42954 BEOU, sub H. alpinum, rev. B., Zlatković 12-Dec-2013); Jažinačko jezero, UTM DM96, livade, tresave, šuma molike (leg. Jovanović, S., Lakušić, D., Bemić, N. 28-Jun-1990, 1510 BEOU-KEGB, sub H. alpigenum, rev. B., Zlatković 12-Dec-2013); Suva reka, UTM DM97, 1400-1900 m, šume (leg. Nikolić, V., Diklić, N., Mladenović, S. 20-Jul-1978, det. N., Diklić, 43556 BEO); Ljuboten, UTM EM07, silikat, 05-Jul-1914, 42693 BEOU); (leg. Košanin, N. 06-Jul-1991, 42798 BEOU); Orman?, UTM EM07, 1400 m (leg. Rudski, I. 16-Jul-1930, 42706 BEOU, sub H. alpinum, rev. B., Zlatković 12Dec-2013); Ošljak, UTM DM97, 1800-2212 m, krečnjak, kamenjar (leg. Nikolić, V., Diklić, N., Bogdanović, M. 19-Jul-1974, det. V., Nikolić, 43547 BEO, sub H. alpinum, rev. B., Zlatković 31-Mar-2014); Piribeg, UTM EM06 (leg. Stevanović, V., Jovanović, S. 21-Jul-1989, 3293 BEOU-KEGB); 1800-2200 m, krečnjak, kamenjar (leg. Nikolić, V., Diklić, N. 21-Jul-1975, det. V., Nikolić, 43545, 43548 BEO, sub H. alpinum, rev. B., Zlatković 31-Mar-2014); Stojkova kuća, UTM EM06, 1200-1800 m, pašnjaci, kamenjari (leg. Nikolić, V., Diklić, N., Mladenović, S. 20-Jul-1977, det. N., Diklić, 43553 BEO); 1600-2400 m (leg. Nikolić, V., Diklić, N., Mladenović, S. 23-Jul-1980, det. N., Diklić, 43554, 43555 BEO); Metohija Peć: Hajla, UTM DN23, $2250 \mathrm{~m}$ (leg. Gošević, S., 22.07., 42700 BEOU, sub H. alpigenum, rev. B., Zlatković 12-Dec2013); Prokletije: Bogićevica, Pasji zub (vrh?), UTM DN21, in pratis alpinis (leg. Černjavski, P., Rudski, I., Lindtner, V. 19-Jul-1933, det. P., Černjavski, 8772 BEO, sub H. alpinum, rev. B., Zlatković 31Mar-2014); Derviš kom, UTM DN20, 2000 m, granit (leg. Černjavski, P., Rudski, I., Lindtner, V. 17-Jul1933, det. P., Černjavski, 8773 BEO, sub H. alpinum, rev. B., Zlatković 31-Mar-2014); (leg. Černjavski, P., Rudski, I., Lindtner, V. 19-Jul-1933, det. P., Černjavski, 8783 BEO, sub H. alpinum, rev. B., Zlatković 31-Mar-2014); Đeravica, UTM DN20, 2000-2650 m, krečnjak, kamenjar (leg. Nikolić, V., Diklić, N., Bogdanović, M. 17-Jul-1973, det. V., Nikolić, 43543 BEO, sub H. alpinum, rev. B., Zlatković 31-Mar2014); Koprivnik, Krš Čvrlje, UTM DN31, 2070 m, serpentinit (leg. Janković, M. 08-Jul-1963, 42943 BEOU, sub H. alpinum, rev. B., Zlatković 12-Dec-2013); Mileševski potok - dolina?, UTM DN32, krečnjak, stene, rub šume (leg. Amidžić, L., 2848 BEOU-KEGB, sub H. maculatum, rev. B., Zlatković 06Feb-2014); Nedžinat, UTM DN22, bukovo-jelova šuma (leg. Černjavski, P., Rudski, I., Lindtner, V. 28-Jul1933, det. P., Černjavski, 8774 BEO, sub H. alpinum, rev. B., Zlatković 31-Mar-2014); 1200-1400 m (leg. Nikolić, V., Diklić, N. 25-Jul-1971, det. N., Diklić, 43542 BEO, sub H. alpinum, rev. B., Zlatković 31Mar-2014); Pločica, UTM DN21, granit (leg. Černjavski, P., Rudski, I., Lindtner, V. 17-Jul-1933, det. P., 
Černjavski, 8780 BEO, sub H. alpinum, rev. B., Zlatković 31-Mar-2014); šuma molike (leg. Černjavski, P., Rudski, I., Lindtner, V. 18-Jul-1933, det. P., Černjavski, 8790 BEO, sub H. barbatum var. trichanthum, rev. B., Zlatković 31-Mar-2014); Žljeb, Maja Rusolija, UTM DN33, krečnjak (leg. Černjavski, P., Rudski, I., Lindtner, V. 02-Aug-1933, det. P., Černjavski, 8784 BEO, sub H. alpinum, rev. B., Zlatković 31Mar-2014); Šar-planina: Bistrica, UTM DM86, Exp. N, 1600-1800 m (leg. Nikolić, V., Diklić, N. 14-Jul1969, det. N., Diklić, 43541 BEO, sub H. alpinum, rev. B., Zlatković 31-Mar-2014); Exp. N, 2000-2200 m, kamenjar (leg. Nikolić, V., Diklić, N., Mladenović, S. 22-Jul-1976, det. N., Diklić, 43552 BEO, sub H. alpinum, rev. B., Zlatković 31-Mar-2014); Crni vrh, UTM DM65 (leg. Rudski, I. 06-Jul-1930, 42709 BEOU, sub H. alpinum); 2100 m (leg. Rudski, I. 05-Jul-1930, 42709 BEOU, sub H. alpinum, rev. B., Zlatković 12-Dec-2013); Kobilica, UTM DM86, 2400 m, pašnjaci, kamenjari (leg. Nikolić, V., Diklić, N., Mladenović, S. 19-Jul-1977, det. N., Diklić, 43559 BEO); 1110 m (leg. Rudski, I. 04-Jul-1930, det. I., Rudski, 8765 BEO, sub H. grisebachii, rev. B., Zlatković 31-Mar-2014); Kobilica - Surduk, UTM DM86, 2200 m, krečnjak, rudine (leg. Duraki, š. 29-Jul-2003, det. Š., Duraki, 976 BEOU-KEGB); Kobilica - Treskavac, UTM DM86, 2300 m, krečnjak i mermer, stene, rudine (leg. Duraki, Š. 01-Jul2003, det. Š., Duraki, 977 BEOU-KEGB); 2100 m, krečnjak, pašnjak (leg. Duraki, Š. 29-Jul-2003, det. Š., Duraki, 978 BEOU-KEGB; 969 BEOU-KEGB, sub H. linarioides, rev. B., Zlatković 06-Feb-2014; 979 BEOU-KEGB, sub H. umbellatum, rev. B., Zlatković 06-Feb-2014); Konjuška, UTM DM96 (leg. Rudski, I. 16-Jul-1930, 42707 BEOU, sub H. alpinum, rev. B., Zlatković 12-Dec-2013).

Hypericum rochelii Griseb. \& Schenk (Fig. 10)

Šumadija Gornjačka klisura, UTM EQ40, krečnjak, stene (leg. Niketić, M., Tomović, G., 18Jun-2004, 18985 BEOU-KEGB); Pomoravlje Paraćin: manastir Ravanica, UTM EP46 (leg. Pančić, J., det. J., Pančić, 3343 BEOU (Herb. Panc.), sub H. alpinum, rev. B., Zlatković 06-Feb-2014); kamenjar(leg. Ilić, Đ. Jun-1908., det. Đ., Ilić, 8788 BEO, sub H. boissieri f. latifolium, rev. B., Zlatković 20-Nov-2014); (leg. Pančić, J., det. J., Pančić, 3403 BEOU (Herb. Panc.), sub H. richeri, rev. B., Zlatković 06-Feb2014); NE Serbia Bor: Rgotski kamen, UTM FP07, kamenjar (leg. Nikolić, V., Diklić, N., Bogdanović, M., 15-Jun-1973, det. V., Nikolić, 43258 BEO, sub H. boissieri, rev. B., Zlatković 20-Nov-2014); Stol, UTM EP99, Exp. SW, kamenjar, Stipa pulcherrima (leg. Nikolić, V., Diklić, N., Bogdanović, M. 29-Jun-1964, det. N., Diklić, 43268 BEO, sub H. boissieri, rev. B., Zlatković 20-Nov-2014); Veliki krš, UTM EP89 (leg. Diklić, N. 14-Jul-1959, det. N., Diklić, 43277 BEO, sub H. boissieri f. latifolium, rev. B., Zlatković 20-Nov-2014); kamenjar (leg. Diklić, N. 29-Jun-1960, det. N., Diklić, 43266, 43267 BEO, sub H. boissieri, rev. B., Zlatković 20-Nov-2014; 43279 BEO, sub H. boissieri f. latifolium, rev. B., Zlatković 20-Nov-2014);

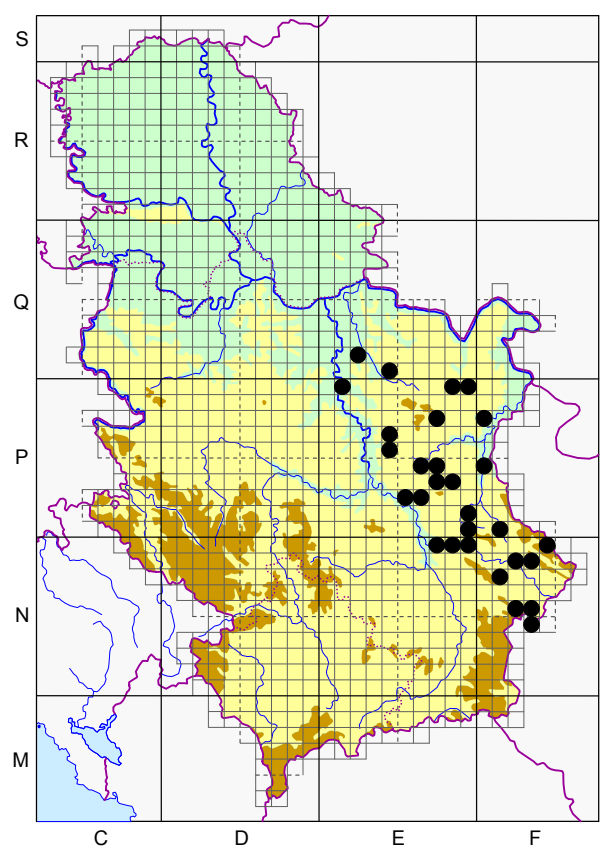

Fig. 10. - Distribution of the species H. rochelii Griseb. \& Schenk in Serbia. pašnjak (leg. Diklić, N. 24-Jun-1961, det. N., Diklić, 43270 BEO, sub H. boissieri, rev. B., Zlatković 20Nov-2014; 43278 BEO, sub H. boissieri f. latifolium, rev. B., Zlatković 20-Nov-2014); (leg. Diklić, N. 22Jun-1962, det. N., Diklić, 43578 BEO); kamenjar (leg. Nikolić, V., Diklić, N. 17-Jun-1972, det. V., Nikolić, 43254 BEO, sub H. boissieri, rev. B., Zlatković 20-Nov-2014); šibljak jorgovana (leg. Nikolić, V., Diklić, N., Bogdanović, M. 17-Jun-1972, det. N., Diklić, 43255, 43256, 43257 BEO, sub H. boissieri, rev. B., Zlatković 20-Nov-2014); 1100 m, kamenjar (leg. Diklić, N. 16-Jul-1959, det. N., Diklić, 43614 BEO, sub H. rumelicum, rev. B., Zlatković 31-Mar-2014); Homolje: Petrovac na Mlavi, UTM EQ21, krečnjak, 
stene, 26-Apr-1951, det. N., Diklić, 43250 BEO, sub H. boissieri, rev. B., Zlatković 20-Nov-2014); Klisura Resave, UTM EP19, krečnjak (leg. Nikolić, V., Diklić, N. 12-Jun-1976, det. N., Diklić, 43245, 43246, 43247, 43248 BEO, sub H. boissieri, rev. B., Zlatković 20-Nov-2014); Zlot, UTM EP77 (leg. Pančić, J., det. J., Pančić, 3413 BEOU (Herb. Panc.), sub H. richeri, rev. B., Zlatković 06-Feb-2014); Lazareva reka - kanjon, UTM EP77 (leg. Stevanović, V. 27-May-1982, 10300 BEOU-KEGB); krečnjak (leg. Lakušić, D., Tomović, G. 22-May-1998, 8970 BEOU-KEGB, sub H. boissieri, rev. B., Zlatković 06-Feb-2014); krečnjak, kamenjari, stene (leg. Stevanović, V. 18-May-1990, 367 BEOU-KEGB, sub H. boissieri, rev. B., Zlatković 12-Dec-2013); 257 m, krečnjak, kamenjari, šibljaci (leg. Vukojičić, S., Jakovljević, K. 22-May-2009, 29644 BEOU-KEGB, sub H. boissieri, rev. B., Zlatković 06-Feb-2014); E Serbia Aleksinac, UTM EP52 (leg. Pančić, J., det. J., Pančić, 3410 BEOU (Herb. Panc.)); Greben, UTM FN34, FN35 (leg. Pančić, J., det. J., Pančić, 3409, 3415 BEOU (Herb. Panc.), sub H. richeri, rev. B., Zlatković 06-Feb-2014); Jerma - klisura: Zvonačka Banja, UTM FN25, stene (leg. Nikolić, V., Diklić, N., ?, det. N., Diklić, 3244 BEO, sub H. boissieri, rev. B., Zlatković 20-Nov-2014); (leg. Nikolić, V., Diklić, N. 18-Jul-1965, det. N., Diklić, 43251 BEO, sub H. boissieri, rev. B., Zlatković 20-Nov-2014); (leg. Nikolić, V., Diklić, N., Rakin, M. 18-Jul-1965, det. N., Diklić, 43275 BEO, sub H. boissieri, rev. B., Zlatković 20-Nov-2014); Knjaževac: Beli Potok, Ždrelo, UTM FP04, krečnjak, stepski fragmenti (leg. Niketić, M., Tomović, G. 04-May-2008, 26930 BEOU-KEGB, sub H. boissieri, rev. B., Zlatković 12-Dec-2013); Kalna, UTM FP10 (leg. Vukojičić, S., Zlatković, B., Ranđelović, V. 08-Jun-1997, 5692 BEOU-KEGB, sub H. boissieri, rev. B., Zlatković 06-Feb-2014); Miljkovac, UTM EP91, 530 m, krečnjak, termofilni pašnjaci, kamenjari (leg. Bogosavljević, S. 2013, det. B., Zlatković, 7247 HMN); Niš, UTM EN79 (leg. Pančić, J., det. J., Pančić, 3336 BEOU (Herb. Panc.)); Ozren: Janior, UTM EP73, pašnjak (leg. Diklić, N. 6.1996, det. N., Diklić, 43272 BEO, sub H. boissieri, rev. B., Zlatković 20-Nov-2014); Leskovik, UTM EP62 (leg. Pančić, J., det. J., Pančić, 3399 BEOU (Herb. Panc.), sub H. richeri, rev. B., Zlatković 06-Feb-2014); Pirot: Jerma - klisura, UTM FN35, 600 m, krečnjak, šibljaci (leg. Stevanović, V., Jovanović, S., Lakušić, D., Jakovljević, K. 26-Jun-2006, 20915 BEOU-KEGB, sub H. boissieri, rev. B., Zlatković 06-Feb-2014); krečnjak, stene, kamenjari (leg. Vukojičić, S., Tomović, G., Mitrović, G., Lazarević, P. 08-Jun-2002, 15017 BEOU-KEGB, sub H. boissieri, rev. B., Zlatković 06-Feb-2014); Kitka, UTM FN38, 1100-1209 m, krečnjak, kamenjar (leg. Nikolić, V., Diklić, N. 19-Jun-1974, det. V., Nikolić, 43261 BEO, sub H. boissieri, rev. B., Zlatković 20-Nov-2014); Rtanj, UTM EP64, EP74 (leg. Pančić, J., det. J., Pančić, 3416, 3417, 3418 BEOU (Herb. Panc.)); (leg. Ranđelović, N. 2010, det. N., Ranđelović, 6578 HMN); Baba, UTM EP45 (leg. Ranđelović, V., Niketić, M., Z. R., Živković, T. 1997, det. V., Ranđelović, 243 HMN); Tumba, UTM EP74, 700-850 m, krečnjak, kamenjari, šibljaci (leg. Niketić, M., Tomović, G. 10-Jul2009, 30200 BEOU-KEGB); Vrmdža, Kusak, UTM EP83, krečnjak, kamenjari, šibljaci (leg. Zlatković, B., Ranđelović, V. 2002, det. B., Zlatković, 7254 HMN); Sićevačka klisura, UTM EN89, EN99, stene (leg. Nikolić, V., Diklić, N. 26-May-1964, det. N., Diklić, 43581 BEO); (leg. Nikolić, V., Diklić, N. 26-Jun-1963, det. N., Diklić, 43577 BEO); (leg. Ranđelović, V., Zlatković, B., det. V., Ranđelović, B., Zlatković, 761 HMN); (leg. Nikolić, V., Diklić, N. 15-Jun-1974, det. V., Nikolić, 43260 BEO, sub H. boissieri, rev. B., Zlatković 20-Nov-2014); manastir Sv. Petka, UTM EN99 (leg. Grebenščikov, O., 43276 BEO, sub H. boissieri, rev. B., Zlatković 20-Nov-2014); krečnjak, stene (leg. Ilić, Đ. Jun-1908., det. Đ., Ilić, 8787 BEO, sub H. boissieri, rev. B., Zlatković 20-Nov-2014); (leg. Nikolić, V., Diklić, N. 25-Jun-1963, det. N., Diklić, 43274 BEO, sub H. boissieri, rev. B., Zlatković 20-Nov-2014); (leg. Diklić, N. 10-Jun-1971, det. N., Diklić, 43264, 43265 BEO, sub H. boissieri, rev. B., Zlatković 20-Nov-2014); krečnjak (leg. Ilić, Đ. 09.07., 8787 BEO, sub H. boissieri f. latifolium, rev. B., Zlatković 20-Nov-2014); (leg. llić, Đ., 8785 BEO, sub H. boissieri rev. B., Zlatković 20-Nov-2014); krečnjak (leg. llić, Đ., 8786 BEO, sub H. boissieri, rev. B., Zlatković 20-Nov-2014); (leg. Nikolić, V., Diklić, N. 26-Jun-1963, det. N., Diklić, 43253 BEO, sub H. boissieri, rev. B., Zlatković 20-Nov-2014); (leg. Nikolić, V., Diklić, N., det. N., Diklić, 43252 BEO, sub H. boissieri, rev. B., Zlatković 20-Nov-2014); (leg. Nikolić, V., Diklić, N., Bogdanović, M. 19-Jun-1975, det. N., Diklić, 43262, 43263 BEO, sub H. boissieri, rev. B., Zlatković 20-Nov-2014); Prosek, UTM EN89, stene (leg. llić, Đ., 8789 BEO, sub H. boissieri, rev. B., Zlatković 20-Nov-2014); Vis, UTM EN79 (leg. Pančić, J., det. J., Pančić, 3334 BEOU (Herb. Panc.)); Sokobanja, UTM EP73, 350 m, 07-Jul-1956, det. N., Diklić, 43243 BEO, sub H. boissieri, rev. B., Zlatković 20-Nov-2014); pašnjak (leg. Diklić, N. 25-Jun1957, det. N., Diklić, 43271 BEO, sub H. boissieri, rev. B., Zlatković 20-Nov-2014); Soko grad, UTM EP73, 329 m, krečnjak (leg. Vukojičić, S., Kuzmanović, N., Jušković, M. 02-Jun-2011, 31997 BEOU-KEGB); krečnjak (leg. Diklić, N. 21-Jun-1957, det. N., Diklić, 43273 BEO, sub H. boissieri, rev. B., Zlatković 20Nov-2014); Stara planina: Oštra kladenac, UTM FN49 (leg. Pančić, J., det. J., Pančić, 3402 BEOU 
(Herb. Panc.), sub H. richeri, rev. B., Zlatković 06-Feb-2014); Svrljiške planine: Pleš, UTM EP90, zajednice Salvia officinalis (leg. Diklić, N., Nikolić, V. 25-May-1964, det. V., Nikolić, 43576 BEO, sub H. boissieri, rev. B.,Zlatković31-Mar-2014); (leg. Pančić, J., det. J., Pančić, 3424 BEOU (Herb. Panc.), sub H. serbicum, rev. B., Zlatković 06-Feb-2014); Ulj kamen, UTM EP90 (leg. Pančić, J., det. J., Pančić, 3407 BEOU (Herb. Panc.), sub H. richeri, rev. B., Zlatković 06-Feb-2014); Vidlič: Crni vrh, UTM FN38, kamenjar (leg. Marković, M. 6.2004, det. M., Marković, $314 \mathrm{HMN}$ ); Četvrti kilometar, UTM FN38, kamenjar, pojas grabićevih šuma (leg. Marković, M. 01-Jun-2008, det. M., Marković, 2750 HMN); Dag Banjica, UTM FN28, kamenjar (leg. Marković, M. 19-Jun-2005, det. M., Marković, 1501 HMN); Ponor, Dupni kamik, UTM FN17, livada (leg. Marković, M. 7.2004, det. B., Zlatković, 192 HMN); Zaječar: Vratarnica, UTM FP04, 540 m, krečnjak, termofilne šume i pašnjaci (leg. Zlatković, B. 2013, det. B., Zlatković, $7257 \mathrm{HMN}$ ).

\section{Hypericum rumeliacum Boiss. subsp. rumeliacum (Fig. 11)}

Banat Deliblatska peščara, UTM EQ07 (leg. Stevanović, V., 1383 BEOU-KEGB); NE Serbia Bor: Brestovac, UTM EP87 (leg. Pančić, J., det. J., Pančić, 3412 BEOU (Herb. Panc.), sub H. richeri, rev. B., Zlatković 06Feb-2014); Brestovačka Banja, UTM EP87 (leg. Diklić, N. 24-Jun-1966, det. N., Diklić, 43613 BEO); C Serbia Prokuplje, UTM EN48 (leg. Lindtner, V., 05-Jul-1953, det. N., Diklić, 43631 BEO); (leg. Jurišić, Ž. 31-May-1911, 8781 BEO, sub H. richeri, rev. B., Zlatković 31-Mar-2014); Hisar, UTM EN48 (leg. Vukojičić, S., Tomović, G., Zlatković, B., Jakovljević, K., Kabaš, E. 08-Jun-2012, 35335 BEOU-KEGB); Viča - Ložđe, UTM EN38, 250 m, šume, Quercus frainetto, Carpinus orientalis (leg. Tomović, G., Vukojičić, S. 25-May-1997, 8345 BEOUKEGB, sub $H$. perforatum subsp. veronense, rev. B., Zlatković 06-Feb-2014); Sokolovica: Rudare, UTM EN26,450 m, andezit, kamenjar, Festuco-Chrysopogonetum grylli (leg. Tomović, G., Niketić, D. 06-Jun-1998, 1312 BEOU-KEGB); (leg. Tomović, G., Vukojičić, S., Ranđelović, V., Zlatković, B. 23-May-1997, 300 BEOU-KEGB); Lubnički krš, UTM EN26, 450 m, andezit, kamenjar, Festuco-Chrysopogonetum grylli, 1312, 300 BEOU); Vidojevica: Sokolica, Bostanište (južna kota 474), UTM EN48, krečnjak (leg. Niketić, M., Tomović, G. 13-Jul-2009, 30265 BEOUKEGB); E Serbia Aleksinac, UTM EP52 (leg. Pančić, J., det. J., Pančić, 3420, 3421, 3422 BEOU (Herb. Panc.); (leg. Perić 1949, 42663 BEOU, sub H. richeri, rev. B., Zlatković 12-Dec-2013); (leg. Pančić, J., det. J., Pančić, 14604 BEOU (Herb. Panc.), sub H. rochelii, rev. B., Zlatković 06-Feb-2014); Kraljevo, UTM EP52, šuma grabića (leg. Nikolić, V., Diklić, N. 13-Jun-1976, det. M., Jovanović, 43642 BEO); Bela Palanka: Crnoklište - Čiflik, UTM FN18, krečnjak, stene (leg. Vukojičić, S., Tomović, G., Mitrović, G., Lazarević, P. 10-Jun-2002, 14935 BEOU-KEGB); Čiflik, UTM FN18, krečnjak, stene (leg. Nikolić, V., Diklić, N. 25-Jun-1978, det. N., Diklić, 43637 BEO); (leg. Nikolić, V., Diklić, N. 25-Jun-1979, det. N., Diklić, 43637 BEO); Šljivovički vrh, kod repetitora, UTM FN17, 1258 m, krečnjak, kamenjar, 997 HMN); Mali Suvodol - Kardašica, UTM FN28, 700 m, krečnjak, kamenjar, 994 HMN); Malo Kurilo, UTM FN08, 388 m, krečnjak, kamenjar, 1000 HMN); Stranje - Barkov del, UTM FN17, 9001200 m, krečnjak, pašnjaci, kamenjari, 999 HMN); Stranje - Dve Ivorke, UTM FN17, 1015 m, krečnjak, kamenjar, 1003 HMN); Šljivovik, UTM FN17, 843 m, krečnjak, kamenjar, 995 HMN); Ždrelo, UTM 
FN17, 588 m, krečnjak, kamenjar, 1001 HMN); Dimitrovgrad: Gosen, kod gradske deponije, UTM FN46, stepski fragmenti (leg. Vukojičić, S., Tomović, G., Zlatković, B. 15-Aug-2003, 17515 BEOU-KEGB); Knjaževac: Beli Potok, UTM FP04 (leg. Pančić, J., det. J., Pančić, 3406 BEOU (Herb. Panc.), sub H. rochelii, rev. B., Zlatković 06-Feb-2014); Bučje, UTM EP93 (leg. Niketić, M., Tomović, G. 28-Jun1998, 11340 BEOU-KEGB); Kulište, UTM EP72, krečnjak, kamenjar (leg. Bogosavljević, S. 2013, det. B., Zlatković, 7246 HMN); Miljkovac, UTM EP91, 602 m, krečnjak, termofilni pašnjaci (leg. Bogosavljević, S. 2013, det. B., Zlatković, 7248 HMN); Svrljiška Topla, UTM EP91, krečnjak, stepski fragmenti (leg. Niketić, M., Tomović, G. 06-Apr-2008, 26989 BEOU-KEGB); Trgoviški Timok klisura, UTM FP02, krečnjak, stene (leg. Stevanović, V., Niketić, M., Tomović, G. 17-Jun-1998, 11921 BEOU-KEGB); Tupižnica, UTM EP94, kamenjari, stene (leg. Niketić, M., Tomović, G. 28-Jun-1998, 11415 BEOU-KEGB); Niš: Gorica, UTM EN79, 03-Jul-1885, 42673 BEOU); Pirot, UTM FN38 (leg. Petrović, S., 42538 BEOU); kamenjari, šibljaci (leg. Nikolić, V., Diklić, N., Bogdanović, M. 20-Jun-1973, det. N., Diklić, 43579, 43580 BEO, sub H. rochelii, rev. B., Zlatković 31-Mar-2014); Carev kladenac, UTM FN38, 600-1000 m, krečnjak, šuma grabića (leg. Nikolić, V., Diklić, N. 16-Jun-1976, det. N., Diklić, 43638 BEO); Krupac, Krupačko blato, UTM FN37, livada (leg. Petrović, B. 2006, det. B., Zlatković, 3266 HMN); Rasnica, UTM FN27, kamenjar (leg. Niketić, M., Tomović, G. 14-Sep-1998, 11167 BEOUKEGB); Sarlak, UTM FN28 (leg. Nikolić, V. 24-May-1974, det. V., Nikolić, 43617 BEO); Rtanj, UTM EP64, EP74 (leg. Pančić, J., det. J., Pančić, 3426 BEOU (Herb. Panc.), sub H. serbicum, rev. B., Zlatković 06-Feb-2014); Sićevačka klisura, UTM EN89, EN99 (leg. Grebenščikov, O. 14-May-1949, det. M., Jovanović, 43593 BEO); Dolac, UTM EN99, zajednice Salvia officinalis (leg. Nikolić, V., Diklić, N. 25-May-1964, det. N., Diklić, 43664 BEO); Kusača, UTM EN89, stene (leg. Nikolić, V., Diklić, N. 26May-1966, det. N., Diklić, 43656, 43657 BEO); Oblik, UTM EN99, 400-800 m, krečnjak (leg. Živković, T. 05-Jul-1997, 5769 BEOU-KEGB, sub H. barbatum, rev. B., Zlatković 06-Feb-2014); Ostrovica manastir Sv. Petka, UTM EN99 (leg. Nikolić, V., Diklić, N. 25-Jun-1963, det. N., Diklić, 43632 BEO); Sićevo, UTM EN89 (leg. Nikolić, V., Diklić, N. 22-May-1964, det. V., Nikolić, 43630, 43633 BEO); (leg. Bornmüller, J. 1888, 42674 BEOU, sub H. richeri, rev. B., Zlatković 12-Dec-2013); Sokobanja, UTM EP73, pašnjak (leg. Diklić, N. 1960, 43615 BEO); (leg. Lindtner, V. 10-Jun-1956, det. N., Diklić, 43665, 43666 BEO); kamenjar (leg. Nikolić, V., Diklić, N., Bogdanović, M. 21-Jun-1973, det. V., Nikolić, 43661 BEO, 43662); Devica, Oštra čuka, UTM EP72, krečnjak, stene (leg. Niketić, M., Tomović, G. 19-Jun2004, 19012 BEOU-KEGB); Oštra čuka, UTM EP73, livade (leg. Diklić, N. 29-Jun-1957, det. N., Diklić, 43612 BEO); (leg. Lindtner, V. 06-Jul-1956, det. N., Diklić, 43608 BEO); Suva planina, 1882, 42537 BEOU); Babušnica, UTM FN16, šuma grabića (leg. Nikolić, V., Diklić, N. 20-Jun-1974, det. V., Nikolić, 43659, 43660 BEO); Devojački grob - Trem, UTM EN98, krečnjak (leg. Vukojičić, S., Tomović, G. 08-Jul-1997, 6309 BEOU-KEGB); Divljana, UTM FN08, 475 m, krečnjak, kamenjar (leg. Jušković, M., Nikolić, B. 26-May-2004, 998 BEOU-KEGB); Divljana - Nerezina, UTM FN08, 475 m, krečnjak, kamenjar, 998 BEOU); Gornja Koritnica, UTM FN07, 600 m, krečnjak, kamenjar, HordeoXeranthemetum annui, $1002 \mathrm{HMN}$ ); 600-800 m, krečnjak, ass. Quercetum frainetto-cerris, $993 \mathrm{HMN}$ ); 535 m, krečnjak, brdske livade, kamenjari, 996 HMN); Koritnička reka - klisura, UTM FN07, 350 m, krečnjak, kamenjar, BEOU); Svrljig, UTM EP90 (leg. Jurišić, Ž. 29-May-1911, det. M., Dimitrijević, 8782 BEO, sub H. richeri, rev. B., Zlatković 31-Mar-2014); Niševac, UTM EP81, 450 m, krečnjak, suvi pašnjaci, kamenjari (leg. Bogosavljević, S. 2013, det. B., Zlatković, 7245 HMN); Kovilovik, UTM EP81, 550 m, krečnjak, kamenjar (leg. Bogosavljević, S. 2013, det. B., Zlatković, 7244 HMN); Svrljiške planine: Crni vrh, UTM FN06, kamenjar (leg. Nikolić, V. 24-Jun-1957, 43625 BEO); Goli vrh, UTM FN09, kamenjar (leg. Nikolić, V. 23-Jun-1957, det. V., Nikolić, 43622 BEO); (leg. Nikolić, V., Diklić, N. 30Jun-1963, det. N., Diklić, 43655 BEO); Pernatica, UTM EP90, pašnjak (leg. Nikolić, V., Diklić, N. 28Jun-1963, det. N., Diklić, 43629 BEO); Pleš, UTM EP90, 820 m, livade, kamenjari, iznad bukovog pojasa (leg. Lindtner, V. 28-Jun-1955, det. N., Diklić, 43628 BEO); kamenjar (leg. Nikolić, V. 26-Jun-1957, det. V., Nikolić, 43621 BEO); livade (leg. Nikolić, V. 27-Jul-1957, 43623, 43624 BEO); (leg. Nikolić, V., Diklić, N. 28-Jun-1963, det. N., Diklić, 43610 BEO); (leg. Pančić, J., det. J., Pančić, 14706 BEOU (Herb. Panc.), sub H. richeri, rev. B., Zlatković 06-Feb-2014); Tumba - Goli vrh, UTM FN09 (leg. Nikolić, V., Diklić, N. 29-Jun-1963, det. N., Diklić, 43654 BEO); Tumba?, UTM FN09 (leg. Nikolić, V., Diklić, N. 29-Jun-1963, det. N., Diklić, 43609 BEO); Žanovački vrh, UTM EP90 (leg. Zlatković, B. 1995, det. B., 
Zlatković, 342 HMN); Vidlič, UTM FN47, FN57, kamenjar (leg. Nikolić, V., Diklić, N. 15-Jun-1974, det. V., Nikolić, 43618, 43619 BEO); Crni vrh, UTM FN38, kamenjar (leg. Marković, M. 6.2004, det. M., Marković, $394 \mathrm{HMN}$ ); pored puteva (leg. Marković, M. 17-May-2008, det. M., Marković, 3902 HMN); kamenjar (leg. Marković, M. 17-Jul-2009, det. M., Marković, 9169 HMN); (leg. Marković, M. 21-May-2005, det. B., Zlatković, $6590 \mathrm{HMN}$ ); Četvrti kilometar, UTM FN38, kamenjar (leg. Marković, M. 07-Jun2005, det. M., Marković, 6920 HMN); (leg. Marković, M. 22-Jun-2008, det. D., Pavlović, 5792 HMN); Dag Banjica, UTM FN28, šibljaci (leg. Marković, M. 24-May-2009, det. M., Marković, 9629 HMN); Gulenovci, UTM FN47, opožarena mesta, kamenjari (leg. Marković, M. 06-Jul-2010, det. M., Marković, 10598 HMN); Izvor, UTM FN37, kamenjar (leg. Marković, M. 05-Aug-2009, det. M., Marković, 9565 HMN); Krupac, UTM FN37, opožarena mesta, kamenjari (leg. Marković, M. 13-Jul-2008, det. D., Pavlović, $5703 \mathrm{HMN}$ ); kamenjar (leg. Marković, M. 24-May-2008, det. M., Marković, 3336 HMN); opožarena mesta, kamenjari (leg. Marković, M. 24-May-2008, det. B., Zlatković, 4102 HMN); kamenjar (leg. Marković, M. 24-May-2008, det. M., Marković, $4672 \mathrm{HMN}$ ); opožarena mesta, kamenjari (leg. Marković, M. 24-May-2008, det. M., Marković, $4759 \mathrm{HMN}$ ); opožarena mesta, hrastove šume (leg. Marković, M., Marković, Lj., Stojanović, Lj., Đorđević, L. 14-Jun-2008, det. M., Marković, 4287 HMN); Vučje, UTM FN37, opožarena mesta, hrastove šume (leg. Marković, M. 05-Aug-2009, det. B., Zlatković, 7197 HMN); opožarena mesta, kamenjari (leg. Marković, M. 05-Aug-2009, det. B., Zlatković, 7212 HMN); kamenjar (leg. Marković, M. 05-Aug-2009, det. B., Zlatković, 7505 HMN); opožarena mesta, kamenjari (leg. Marković, M. 06-Jun-2009, det. B., Zlatković, 7548 HMN); (leg. Marković, M., Petrović, B. Jotić, B., Marković, Lj. 06-Jun-2009, det. B., Zlatković, 7111 HMN); kamenjar (leg. Marković, M., Petrović, B. Jotić, B., Marković, Lj. 06-Jun-2009, det. B., Zlatković, 7175 HMN); Mali vrh, UTM FN38, kamenjar (leg. Marković, M. 29-May-2005, det. M., Marković, $1350 \mathrm{HMN}$ ); Odorovački kamen - Golemi vrh, UTM FN47, krečnjak (leg. Vukojičić, S., Tomović, G., Zlatković, B. 14-Aug-2003, 17451 BEOU-KEGB); Rsovci, UTM FN48, kamenjar (leg. Marković, M. 08-Jun-2008, det. M., Marković, 3530 HMN); stene (leg. Marković, M. 12-Jun-2004, det. M., Marković, 932 HMN); Tepoš, UTM FN47, pored puteva (leg. Marković, M. 25-Jul-2005, det. M., Marković, 1603 HMN); Vazganica, UTM FN38, šumski put (leg. Marković, M. 06-Jul-2009, det. M., Marković, 9907 HMN); VIkovijski kamik, UTM FN57, kamenjar (leg. Marković, M. 17-May-2009, det. M., Marković, 9451 HMN); Vučje, UTM FN37, opožarena mesta, hrastove šume (leg. Marković, M. 03-Jul-2010, det. M., Marković, 10471 HMN); (leg. Marković, M. 06-Jun2010, det. Marković, M., Jotić, B., Fusijanović, I., Marković, Lj., 10519 HMN); opožarena mesta, kamenjari (leg. Marković, M., Jotić, B., Fusijanović, I., Marković, Lj. 06-Jun-2010, det. M., Marković, 10691 HMN); Zabrđe, UTM FN46, livada (leg. Marković, M. 12-Jun-2005, det. M., Marković, 1302 HMN); $\underline{\mathbf{S}}$ Serbia Bujanovac: Končuljska klisura, UTM EN50 (leg. Nikolić, V., 27-Jun-1965, det. V., Nikolić, 43604, 43605 BEO); (leg. Nikolić, V. 28-Jun-1965, 43606 BEO); (leg. Nikolić, V. 29-Jun-1965, 43602 BEO); kamenjar (leg. Nikolić, V. 02-Jun-1966, det. V., Nikolić, 43597, 43598, 43599 BEO); (leg. Nikolić, V., Diklić, N. 16-Jun-1975, det. V., Nikolić, 43626 BEO); (leg. Nikolić, V., Diklić, N., Bogdanović, M. 18-Jul-1874, det. V., Nikolić, 43627 BEO); (leg. Jurišić, Ž. 10-May-1914, 8744 BEO, sub H. richeri, rev. B., Zlatković 31Mar-2014); Lopardince, UTM EN60, kamenjar (leg. Diklić, N. 01-Jun-1963, det. N., Diklić, 43607 BEO); Lučane, UTM EM59, hrastova šuma (leg. Nikolić, V. 06-Jun-1967, det. V., Nikolić, 43601 BEO); Pljačkovica, Še-Arap, UTM EN71 (leg. llić, Đ. 1910, 8740 BEO); Grdelička klisura: Momin kamen, UTM EN82, 390 m, andezit, termofilne šume (leg. Đorđević, A., 2010, det. B., Zlatković, 7251 HMN); Leskovac: Belanovce, UTM EN66 (leg. Ilić, Đ., 42672 BEOU, sub H. richeri, rev. B., Zlatković 12-Dec-2013); Preševo, UTM EM58, livade (leg. Diklić, N. 31-May-1963, det. N., Diklić, 43611 BEO); (leg. Jovanović, V. 1984, det. B., Zlatković, 4800 HMN); krečnjak, kamenjar, pojas grabićevih šuma (leg. Nikolić, V., Diklić, N. 16-May-1975, det. V., Nikolić, 43663 BEO); Radan, UTM EN45, EN46, livada (leg. Jovanović, v. 1976, det. V., Jovanović, 5599 HMN); Sokolov vis, Veliki krš, UTM EN35, silikat, stene (leg. Niketić, M., Tomović, G. 01-May-2008, 26897 BEOU-KEGB); Vranje, UTM EN71 (leg. Nikolić, V. 26-Jul-1962, det. M., Jovanović, 43640 BEO); (leg. Pančić, J., det. J., Pančić, 3423 BEOU (Herb. Panc.)); Končuljska klisura, UTM EN50, kamenjar (leg. Nikolić, V. 07-Jun-1967, det. V., Nikolić, 43600 BEO); Neradovac - Ribince, UTM EN70, obradive površine, vinogradi (leg. Stevanović, V., Vukojičić, S., Tomović, G., Zlatković, B. 16-Jun-2003, 16988 BEOU-KEGB); Pljačkovica, UTM EN71 (leg. Ilić, Đ. 1910, 8739 BEO); SE Serbia Rudina, UTM FN20 (leg. Milosavljević, V., 
Ranđelović, N. 2003, det. N., Ranđelović, $2278 \mathrm{HMN}$ ); 900-1200 m, krečnjak, stene, kamenjari (leg. Niketić, M., Tomović, G., Zlatković, B., Anačkov, G. 16-Aug-2006, 22681 BEOU-KEGB); (leg. Ranđelović, N. 2002, det. N., Ranđelović, 2406 HMN); (leg. Ranđelović, N. 2004, det. N., Ranđelović, 2190, 2213, 2220 HMN); Trgovište, UTM EM88 (leg. Tomović, G., Ranđelović, V., Zlatković, B. 08-Aug-1997, 8095 BEOUKEGB, sub H. barbatum, rev. B., Zlatković 06-Feb-2014); Vlasina: Mala Plana, UTM FN13 (leg. Pančić, J., det. J., Pančić, 3414 BEOU (Herb. Panc.), sub H. richeri, rev. B., Zlatković 06-Feb-2014); Vranje: Zlatokop, UTM EN70, obradive površine, vinogradi (leg. Stevanović, V., Vukojičić, S., Tomović, G., Zlatković, B. 16-Jun-2003, 17016 BEOU-KEGB); Kosovo Gnjilane: Kmetovce - Dobrovčane, UTM EN40 (leg. Nikolić, V., 26-Jun-1966, 43603 BEO); Novo Brdo, UTM EN31 (leg. Tomović, G., Ranđelović, V., Zlatković, B. 08-Aug-1997, 8147 BEOU-KEGB); Priština, UTM EN12 (leg. Petrović, J. 14Jun-1909, BEOU); vinogradi (leg. Jurišić, Ž. 08-May-1914, 8768 BEO, sub H. richeri, rev. B., Zlatković 31-Mar-2014); Metohija Paštrik, UTM DM67 (leg. Košanin, N., 17-Jul-2013, 42555 BEOU); stene (leg. Košanin, N. 17-Aug-1919, 42540 BEOU); Gorožup, UTM DM66, 300-500 m, krečnjak, šikare, Quercetum macedonicae (leg. Nikolić, V., Diklić, N., Mladenović, S. 25-Jul-1979, 43634 BEO); Prizren, UTM DM77 (leg. Košanin, N. 05-May-2013, 42556 BEOU); Prizrenska Bistrica - klisura, UTM DM87 (leg. Košanin, N. 05-May-1913, 42557 BEOU); (leg. Soška, Th. 10-Jul-1914, BEOU).

Hypericum umbellatum A. Kerner (Fig. 12)

C Serbia Kopaonik: Treska, UTM DN98, serpentinit (leg. Niketić, M., Tomović, G., Novčić, R., 09-Jul-2005, 19930 BEOU-KEGB); E Serbia Rtanj, UTM EP64, EP74 (leg. Pančić, J., det. J., Pančić, 3435, 3434, 3436 BEOU (Herb. Panc.)); (leg. Soška, Th. 6.1924, 42680 BEOU); (leg. Pančić, J., det. J., Pančić, 3361 BEOU (Herb. Panc.), sub H. ciliatum, rev. B., Zlatković 06Feb-2014); (3404 BEOU (Herb. Panc.), sub $H$. richeri, rev. B., Zlatković 06-Feb-2014); 1400 m (leg. Grebenščikov, O. 15-Aug-1941, det. O., Grebenščikov, 8763 BEO); Šiljak - Ledeničke strane, UTM EP74, Exp. E, 1100-1550 m, pašnjaci (leg. Diklić, N., Nikolić, V. 29-Jun-1971, det. N., Diklić, 43590 BEO); Stara planina: Midžor, UTM FP30 (leg. Pelivanović, S. 9.1884, 43088 BEOU); Topli Do, UTM FP30, $1100 \mathrm{~m}$ (leg. Grebenščikov, O. 05-Jul-1946, det. O., Grebenščikov, 8764 BEO); Žarkova čuka, UTM FP30, crveni peščari i konglomerati, planinski pašnjaci (leg. Mihailović, T. 26-Jul-1993, det. T., Mihailović, 480 BEOU-KEGB); Suva planina (leg. D. M. 1879, 42679 BEOU); (leg. Ranđelović, V. 1989, det. V., Ranđelović, 1033 HMN); Sokolov kamen, UTM EN98 (leg. Ranđelović, V. 1992, det. V., Ranđelović, 1637 HMN); Trem, UTM EN98, 1700 m, krečnjak,

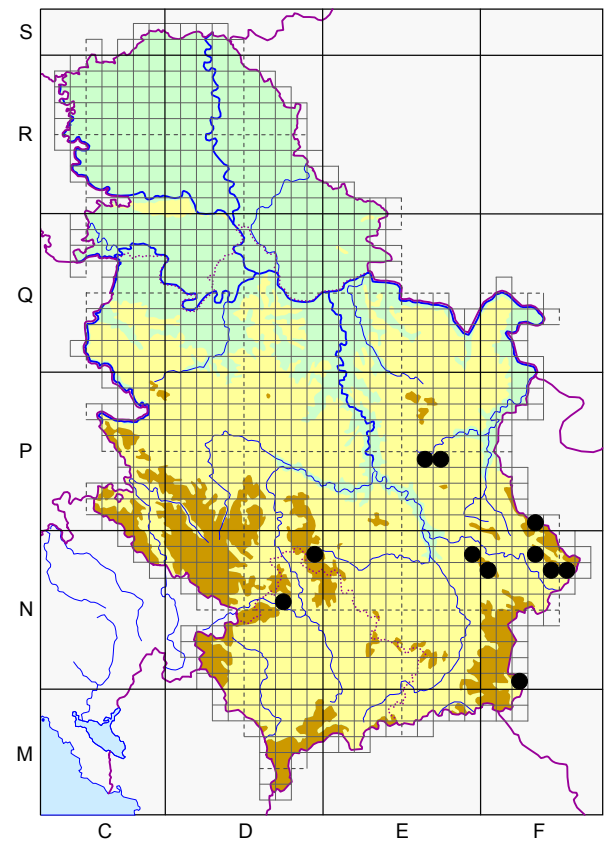

Fig. 12. - Distribution of the species H. umbellatum A. Kerner in Serbia. sipari (leg. Zlatković, B. 2001, det. B., Zlatković, 7265 HMN); 1500 m, krečnjak, kamenjar (leg. Nikolić, V., Diklić, N., Mladenović, S. 26-Jul-1977, det. V., Nikolić, 43592 BEO); Trem (Golemi kamen), UTM EN98, 1100 m, krečnjak (leg. Zlatković, B. 1993, det. B., Zlatković, 7262 HMN); Valožje, UTM FN07, 1750 m, krečnjak, vrtače (leg. Zlatković, B. 1996, det. B., Zlatković, 7264 HMN); Vidlič, UTM FN47, FN57 (leg. Ranđelović, N. 2010, det. N., Ranđelović, 6579 HMN); Basarski kamik, UTM FN38, livada (leg. Blagojevic, I. 2009, det. N., Ranđelović, 6637 HMN); SE Serbia Rudina, UTM FN20, krečnjak, pašnjaci (leg. Ranđelović, N., 1987, det. N., Ranđelović, $521 \mathrm{HMN}$ ); Kosovo Zubin Potok: Pridverica - jezero, UTM DN75 (leg. Pančić, J., det. J., Pančić, 3433 BEOU (Herb. Panc.)). 


\section{H. sect. OLIGostema (Boiss.) Stef.}

Hypericum humifusum L. subsp. humifusum (Fig. 13)

W Serbia Užice: Jelova gora, UTM DP06 (leg. Lakušić, D., Vukojičić, S., 25-Jul-1999, 9259 BEOU-KEGB).

\section{H. sect. HIRTELLA Stef.}

\section{Hypericum hyssopifolium Chaix} (Fig. 14)

NE Serbia Bor: Stol, UTM EP99 (leg. Pančić, J., det. J., Pančić, 3374 BEOU (Herb. Panc.)); Veliki krš, UTM EP89, 1149 m, kamenjar (leg. N. B. 13-Jul-1990, 1705 BEOU-KEGB).

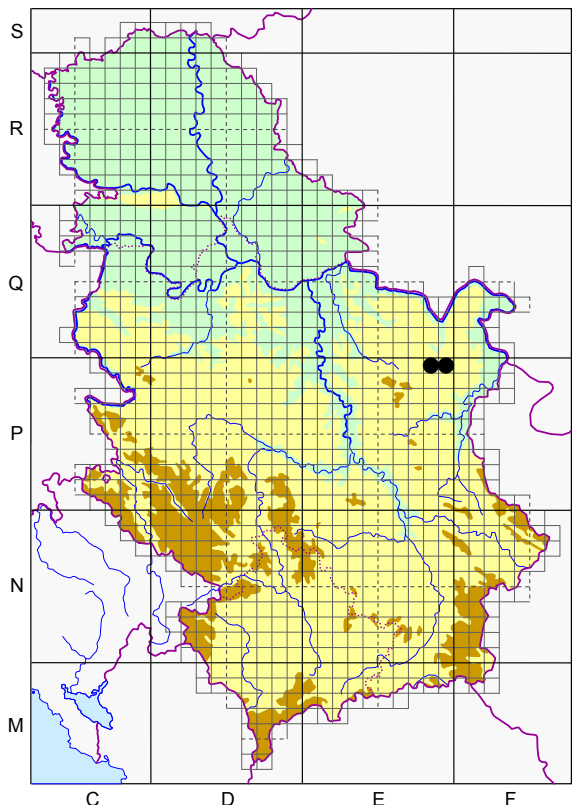

Fig. 14. - Distribution of the species H. hyssopifolium Chaix in Serbia.

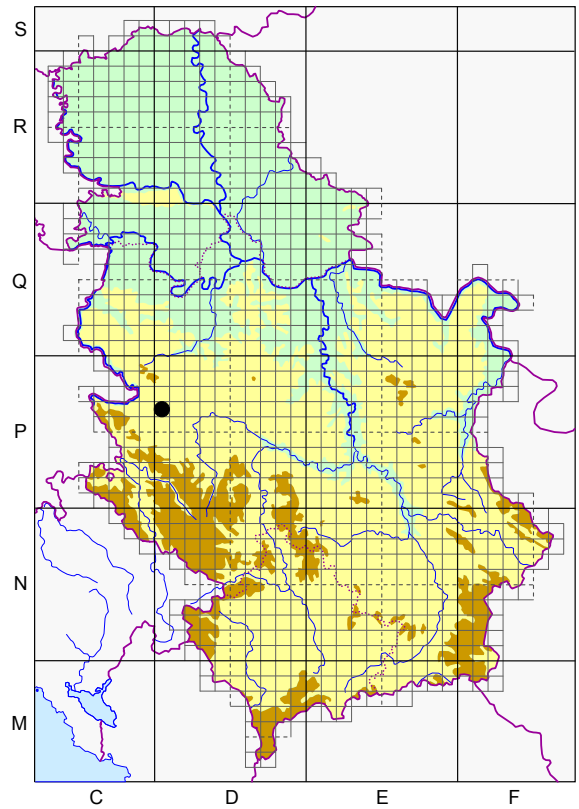

Fig. 13. - Distribution of the species H. humifusum L. in Serbia.

\section{H. sect. TAENIOCARPIUM Jaub. \& Spach}

Hypericum hirsutum L. (Fig. 15)

Banat Vršačke planine: Široko bilo, UTM EQ39, šume (leg. Nikolić, V., Diklić, N., 26-Jun1968, det. N., Diklić, 43288 BEO); Srem Fruška gora: Ledinci, Ledinačko jezero, UTM DR00, šume (leg. Jovanović, M., 27-Jun-2003, det. M., Jovanović, 43315, 43316, 43317 BEO); NW Serbia Gučevo: Crni vrh - Velika ravan, UTM CQ52 (leg. Niketić, M., Tomović, G., 25-Apr2008, 26700 BEOU-KEGB); Maljen: Divčiba-

re, UTM DP18 (leg. Popović, M. 25-Jun-1950, 42894 BEOU); Nova Varoš: Akmačići, Uvac kanjon, UTM DP00, 1000 m, krečnjak, kamenjar (leg. Nikolić, V., Diklić, N. 10-Jul-1977, det. M., Jovanović, 43302, 43303, 43304 BEO);Veliki Oštrik, UTM CP91, 1000-1200 m, krečnjak, stene, rudine (leg. Niketić, M., Tomović, G. 14-Aug-2008, 28048 BEOU-KEGB); Povlen: Veliki Povlen, UTM CP98, 1100 m, krečnjak, šume, Ostrya carpinifolia (leg. Niketić, M., Tomović, G. 11-Jun-2011, 32218 BEOUKEGB); Šumadija Avala, UTM DQ64, šuma, 18-Apr-1951, 42946 BEOU); (leg. V. P. 07-Jun-1952, 42945 BEOU); Beograd (leg. Jovanović, S. 1989, BEOU-KEGB); Košutnjak, UTM DQ55 (leg. Lakušić, D. 10-Apr-1986, 946 BEOU-KEGB); Rakovica, UTM DQ55 (leg. Janković, M. 24-Jun-1953, 42955 BEOU); Ripanj, UTM DQ64 (leg. Bornmüller, J. 1888, BEOU); Višnjica, UTM DQ66 (leg. 


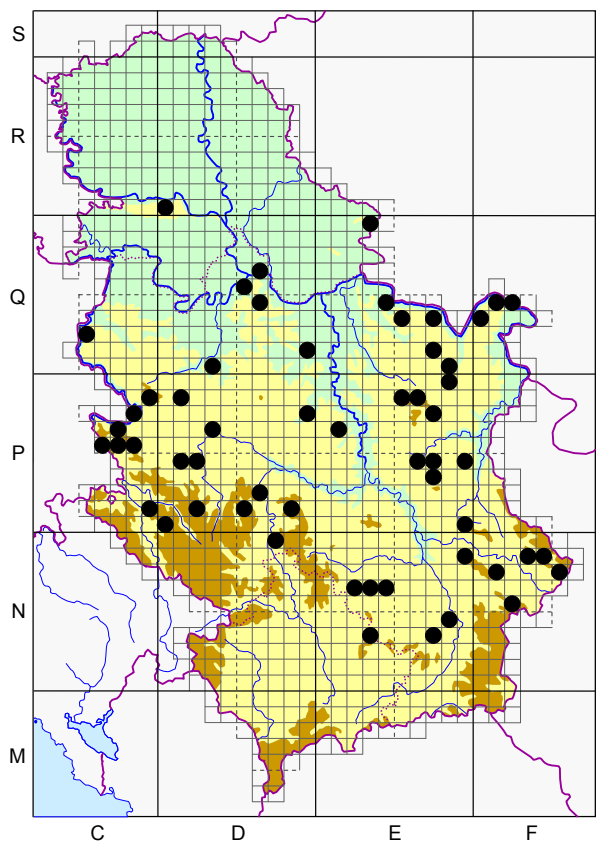

Fig. 15. - Distribution of the species H. hirsutum L. in Serbia.
Bogojević, R., 42921 BEOU); Kragujevac: Balaševačka šuma, UTM DP97 (leg. Rudski, I. 14-Jul-1936, det. I., Rudski, 8684 BEO); Beloševac, UTM DP97, šume (leg. Rudski, I. 14-Jul-1993, det. M., Jovanović, 43308, 43309 BEO); Bresnica - dolina, ul. Mile i Rajka Stojadinovića, UTM DP97, 170 m, ruderalne površine (leg. Marinković, D. 07-Aug-1998, det. V., Stevanović, BEOU-KEGB); Mečkovac, UTM DP97 (leg. Rudski, I. 29-Aug-1939, det. I., Rudski, 8685 BEO); Pomoravlje Jagodina: Belica, UTM EP16 (leg. Pančić, J., det. J., Pančić, 3368, 3369 BEOU (Herb. Panc.)); Lajkovac: manastir Bogovađa, UTM DQ30, šume (leg. Černjavski, P. 06-Jul-1942, det. P., Černjavski, 8683 BEO); Vračevići, UTM DQ30, šuma graba (leg. Černjavski, P. 08-Jul1942, det. P., Černjavski, 8682 BEO); Smederevska Palanka: Kudreč - jezero, UTM DQ91, šume (leg. Jovanović, M. 03-Jul-2003, det. M., Jovanović, 43314 BEO); NE Serbia Beljanica, UTM EP58, EP68 (leg. Pančić, J., det. J., Pančić, 3373 BEOU (Herb. Panc.)); Bor: Veliki krš, UTM EP89 (leg. N. B. 07-Jun1991, 1463 BEOU-KEGB); Donji Milanovac: Poreč, UTM EQ80, šume (leg. Nikolić, V., Diklić, N. 02-Aug-1966, det. N., Diklić, 43299 BEO);

Đerdap: Debelo Brdo, UTM EQ53, krečnjak (leg. Nikolić, V., Diklić, N., Mladenović, S. 26-Jun-1980, det. M., Jovanović, 43305 BEO); Golubac, UTM EQ44, pored puteva (leg. Nikolić, V., Diklić, N. 22-Jul-1967, det. N., Diklić, 43295 BEO); Pesača - potok, UTM EQ73, bukova šuma (leg. Nikolić, V., Diklić, N. 28Jun-1967, det. N., Diklić, 43297 BEO); Ploče - Veliki Štrbac, UTM FQ03, krečnjak, polidominatna šuma (leg. Stevanović, V., Vukojičić, S., Tomović, G. 11-Jul-2001, 13233 BEOU-KEGB, sub H. atomarium, rev. B., Zlatković 06-Feb-2014); Tekija, UTM FQ14, livade (leg. Nikolić, V., Diklić, N. 27-Jul-1967, det. N., Diklić, 43298 BEO); Veliki Štrbac, Pecka Bara, UTM FQ03, pored puteva (leg. Nikolić, V., Diklić, N. 25-Jun-1967, det. N., Diklić, 43296 BEO); Kladovo, UTM FQ24 (leg. Pančić, J., det. J., Pančić, 14705 BEOU (Herb. Panc.)); Majdanpek: Debeli Lug, UTM EQ71, šuma, pored potoka (leg. Niketić, M., Tomović, G. 08-Jul-2009, 30143 BEOU-KEGB); Pek - dolina, UTM EQ71 (leg. Černjavski, P. 22-Jul1946, det. P., Černjavski, 8690 BEO); Zlot: Malinik, UTM EP77, 800 m, krečnjak (leg. Lakušić, D. 16Jul-1986, 335 BEOU-KEGB); W Serbia Čemerno: Savošnica - dolina, Klisura, UTM DP51 (leg. Stevanović, V., Niketić, M., Vukojičić, S., Tomović, G., 01-May-2004, 18624 BEOU-KEGB); Javor: Kušići - Deretin, Visioc, UTM DP21, 800 m, krečnjak, bukova šuma (leg. Tomović, G. 24-Jul-1996, det. M., Niketić, 1801 BEOU-KEGB); Ovčarsko-Kablarska klisura: Kablar, UTM DP36, pored puteva (leg. Jovanović, M., Niketić, M. 20-Jul-2004, det. M., Jovanović, 43311, 43312, 43313 BEO); Tara, UTM CP76, CP75, serpentinit, opožarena šumska staništa (leg. Stevanović, V., Jovanović, S., Vukojičić, S. 10-Jun-2000, 14329 BEOU-KEGB); Greben, UTM CP76 (leg. Menković, N. 16-Jul-2000, 10684 BEOUKEGB); Kremna, UTM CP85, borove šume (leg. Diklić, N. 16-Sep-1966, det. N., Diklić, 43294 BEO); Rača, UTM CP87, bukovo-jelova šuma (leg. Diklić, N., Rakin, M. 17-Jul-1963, det. N., Diklić, 43287 BEO); čistine u bukovo-jelovoj šumi (leg. Diklić, N., Rakin, M. 17-Jul-1963, det. N., Diklić, 43293 BEO); Stolac, UTM CP75, 1200 m (leg. Stevanović, V., Jovanović, S., Vukojičić, S. 14-Jul-2000, 14572 BEOUKEGB); Zaovine, UTM CP65 (leg. Pančić, J., det. J., Pančić, 3367 BEOU (Herb. Panc.)); pašnjak, obradive površine (leg. Stevanović, V., Jovanović, S. 11-Sep-1996, 2963 BEOU-KEGB); Veliki Rzav klisura, UTM DP24, DP14, šuma, ass. Oxali-Alnetum incanae (leg. Lakušić, D., Jovanovć, S. 29-Jul1990, 2249 BEOU-KEGB); Zvezda: Veliki Stolac, UTM CP75 (leg. Menković, N. 21-Jul-2000, 10669 BEOU-KEGB); C Serbia Goč, UTM DP81, 1500 m (leg. Niketić, M., 19-Jul-1925, det. M., Niketić, 8686 BEO); Goljak: Mrkonjski vis, UTM EN33 (leg. Jovanović, V. 1983, det. M., Marković, 4525 
HMN); Kopaonik (leg. Lakušić, D., BEOU-KEGB); Semeteško jezero, UTM DN79 (leg. Pančić, J., det. J., Pančić, 3370 BEOU (Herb. Panc.)); Sokolovica: Krompirište, UTM EN26, 800 m, andezit, kamenjari, stene, 1706 BEOU); Presla - Krompirište, UTM EN26, 800 m, andezit, kamenjari, stene (leg. Tomović, G., Niketić, D. 17-Jul-2000, 1706 BEOU-KEGB); Radulovac, UTM EN36, 1050 m, andezit, pored puta, Fagetum moesiacae montanum, 1175 BEOU); (leg. Tomović, G., Niketić, D. 02-May1998, 1175 BEOU-KEGB); Stolovi, UTM DP62 (leg. Pančić, J., det. J., Pančić, 3371 BEOU (Herb. Panc.)); E Serbia Bela Palanka: Šljivovički vrh, Stranje - Barkov del, UTM FN17, 900-1200 m, krečnjak, kamenjar, 987 BEOU); (leg. Jušković, M., Zlatković, B. 07-Jul-2004, 987 BEOU-KEGB); Jerma - klisura: Zvonačka Banja, UTM FN25, 1100-1300 m, kamenjar (leg. Nikolić, V., Diklić, N. 17-Jul1965, det. N., Diklić, 43280 BEO); livade (leg. Nikolić, V., Diklić, N., Rakin, M. 15-Jul-1965, det. N., Diklić, 43290 BEO); Knjaževac: Tupižnica, UTM EP94, stene, kamenjari (leg. Niketić, M., Tomović, G. 28Jun-1998, 11432 BEOU-KEGB); Rtanj, UTM EP64, EP74 (leg. Pančić, J., det. J., Pančić, 3372 BEOU (Herb. Panc.)); UTM EP74, 1100 m, krečnjak, šikare (leg. Diklić, N., Mladenović, S. 19-Jul-1988, det. M., Jovanović, 43300, 43306, 43307 BEO); Sokobanja, UTM EP73, pored puteva (leg. Diklić, N. 24-Jul1957, det. N., Diklić, 43291 BEO); šume (leg. Diklić, N. 31-Jul-1963, det. N., Diklić, 43292 BEO); Stara planina: Slavinja, UTM FN57, planinski pašnjaci (leg. Marković, M. 8.2004, det. M., Marković, 1046 HMN); Suva planina: Bojanine Vode, UTM EN98, bukova šuma (leg. Marković, M. 2005, det. B., Zlatković, 3151 HMN); Devojački grob - Trem, UTM EN98, krečnjak (leg. Vukojičić, S., Tomović, G. 08-Jul-1997, 6334 BEOU-KEGB); Mosor - Sokolov kamen, UTM EN98, krečnjak (leg. Vukojičić, S., Tomović, G. 06-Jul-1997, 6659 BEOU-KEGB); Svrljig: Niševac, UTM EP90 (leg. Dimitrijević, M. M. 29May-1911, det. Ž., Jurišić, 8691 BEO); Vidlič: Basarski kamik, planinarski dom, UTM FN38, šumske progale (leg. Marković, M. 27-Jul-2008, det. D., Pavlović, 6055 HMN); Mojinci, UTM FN57, planinske livade (leg. Marković, M. 11-Jun-2005, det. D., Pavlović, 1922 HMN); Ponor, UTM FN17, livada (leg. Marković, M. 7.2004, det. M., Marković, 12 HMN); Vazganica, UTM FN38, šumski put (leg. Marković, M. 17-Jul-2008, det. M., Marković, 5653 HMN); šuma (leg. Marković, M., Pavlović, D., Marković, Lj. 21-Jun-2008, det. D., Pavlović, 3674 HMN); Vazganica - Rsovci, UTM FN48, planinski pašnjaci (leg. Marković, M., Andrejić, Z. 31-Jul-2004, det. M., Marković, 1173 HMN); S Serbia Kukavica, UTM EN73, EN84 (leg. Jovanović, V., 1971-1974, det. V., Jovanović, 5596 HMN); Jasićko Ravnište, UTM EN73 (leg. Jovanović, V. 1973, det. V., Jovanović, 5597 HMN); Pekarka, UTM EN73 (leg. Jovanović, V. 1973, det. V., Jovanović, $5598 \mathrm{HMN}$ ); Radan: Petrova gora, UTM EN46, bukova šuma (leg. Nikolić, V. 24-Jul-1959, det. M., Jovanović, 43301 $\mathrm{BEO})$.

\section{Hypericum linarioides Bosse} (Fig. 16)

E Serbia Stara planina: KIjun, UTM FP12 (leg. Pančić, J., det. J., Pančić, 3398 BEOU (Herb. Panc.), sub H. repens, rev. B., Zlatković 06-Feb2014); Vrtibog, UTM FN49, 1500 m, krečnjak, kamenjar (leg. Niketić, M. 04-Aug-1993., det. M. Niketić, BEO); Suva planina, 1882, 42656, 1887, 42653 BEOU); (leg. Dimitrijević, M. M. 06-Jul-1911, det. Ž., Jurišić, 8622 BEO, sub H. repens, sub H. repens, rev. B., Zlatković 06Feb-2014); Devojački grob - Trem, UTM EN98, krečnjak (leg. Vukojičić, S., Tomović, G. 08-Jul-1997, 6349 BEOU-KEGB); Trem, UTM EN98, 1800 m, krečnjak, kamenjar (leg. Bogosavljević, S. 2013, det. B., Zlatković, 7253 HMN); (leg. Pančić, J., det. J.,Pančić, 3397 BEOU (Herb.

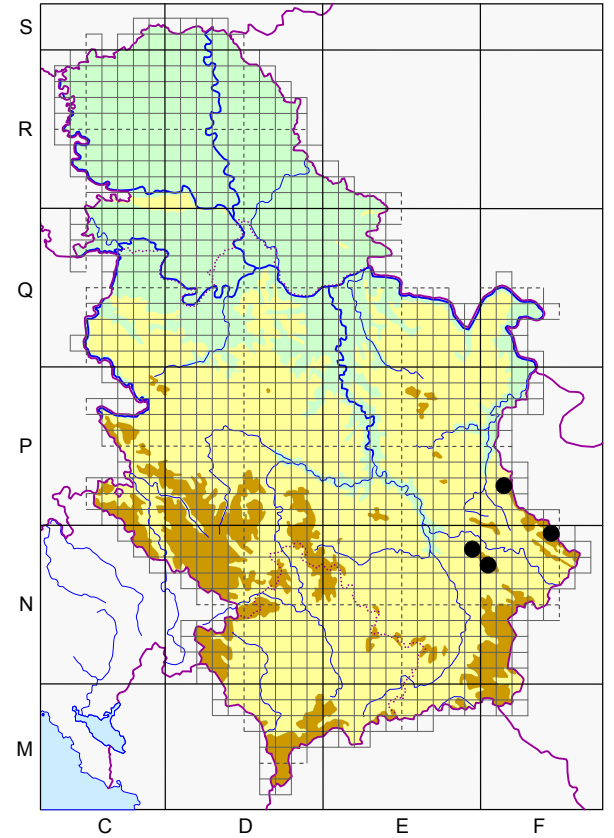

Fig. 16. - Distribution of the species $H$. linarioides Bosse in Serbia. 
Panc.), sub H. repens, rev. B., Zlatković 06-Feb-2014); Valožje, UTM FN07, 1750 m, krečnjak (leg. Zlatković, B. 1993, det. B., Zlatković, 7263 HMN); Svrljiške planine: Zeleni vrh, UTM FN09, 1300 m, krečnjak, kamenjar (leg. Niketić, M. 02-Jun-2008., det. M. Niketić, BEO).

\section{H. sect. ADENOSEPALUM Spach}

\section{Hypericum annulatum Moris subsp. annulatum (Fig. 17)}

NE Serbia Kučaj: Strački vrh?, UTM EP67 (leg. Pelivanović, S., 8.1884, 42835 BEOU, sub H. atomarium, rev. B., Zlatković 12-Dec2013); kalcit (leg. Pelivanović, S. 8.1884, 42835 BEOU, sub H. lanuginosum, rev. B., Zlatković 12-Dec-2013); C Serbia Kopaonik (leg. Pančić, J., det. J., Pančić, 3379 BEOU (Herb. Panc.), sub H. olympicum, rev. B., Zlatković 06-Feb2014); Drenje, Drenjska klisura, UTM DP80, 850 m, granodiorit, kamenjari, Festuca sp. (leg. Lakušić, D., Niketić, M. 18-Apr-1986, det. D., Lakušić, BEOU-KEGB, sub H. atomarium subsp. degenii, rev. B., Zlatković 06-Feb-2014); Treska, UTM DN98, 42534 BEOU); Prokuplje: Markov kamen, UTM EN58 (leg. Jurišić, ž. 01-Jul-1911, det. Ž., Jurišić, 8688, 8687 BEO, sub H. hirsutum, rev. B.,Zlatković31-Mar-2014); Sokolovica: Ravan - Aleksino brdo, UTM EN36, $650 \mathrm{~m}$, andezit, ass. Querco-Carpinetum orientalis silicicolum, 1079 BEOU); (leg. Tomović, G., Niketić, D. 03-Apr-1998, 1079 BEOU-KEGB, sub H. atomarium, rev. B., Zlatković 06-Feb-2014); E Serbia Niš, UTM EN79 (leg. Pančić, J., det. J., Pančić, 3335 BEOU (Herb. Panc.)); Matejevac, UTM EP70 (leg. Pančić, J., det. J., Pančić, 3346 BEOU (Herb.

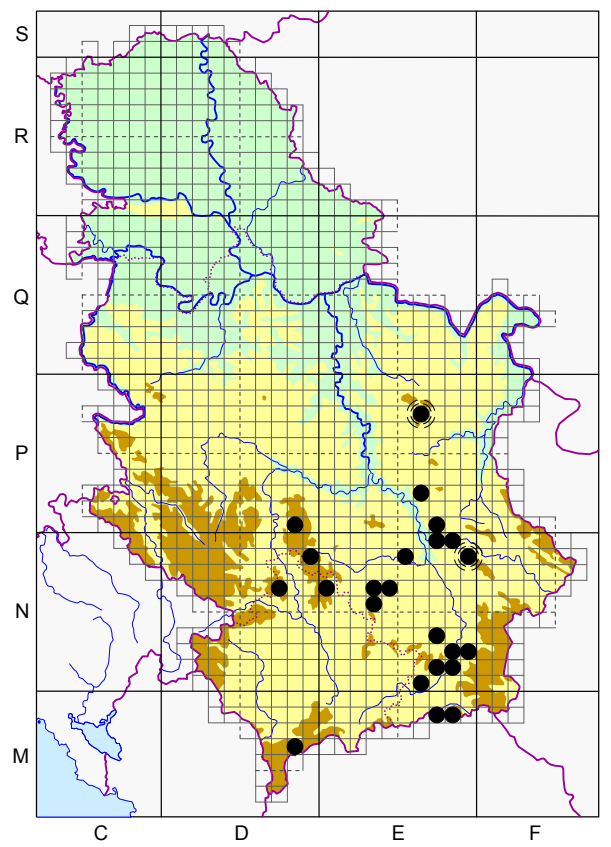

Fig. 17. - Distribution of the species H. annulatum Moris in Serbia.

Panc.), sub H. atomarium, rev. B., Zlatković

06-Feb-2014); Niška Banja, UTM EN89, šume (leg. Petrović, S. 1879, 42523 BEOU, sub H. atomarium, rev. B., Zlatković 12-Dec-2013); Ozren: Leskovik, UTM EP62 (leg. Pančić, J., det. J., Pančić, 3347 BEOU (Herb. Panc.), sub H. atomarium, rev. B., Zlatković 06-Feb-2014); Sićevačka klisura: Prosek, UTM EN89, krečnjak, belograbićeva šuma (leg. Menković, N., Tasić, S. 19-Jun-1998, 14911 BEOUKEGB, sub H. atomarium subsp. degenii, rev. B., Zlatković 06-Feb-2014); Suva planina, UTM EN98 (leg. D. M. 1879, BEOU); SW Serbia Rogozna: Grižani Han, UTM DN76, serpentinit, brdski pašnjaci (leg. Krivošej, Z., Prodanović, D., 13-Jul-2010, 39366 BEOU-KEGB); S Serbia Bujanovac: Končuljska klisura, UTM EN60 (leg. Nikolić, V., 02-Jun-1965, det. V., Nikolić, 43188 BEO, sub H. atomarium subsp. degenii, rev.?); (leg. Nikolić, V., 02-Jun-1965, det. V., Nikolić, 43189, 43190 BEO, sub H. atomarium subsp. degenii, rev. B., Zlatković 31-Mar-2014); (leg. Nikolić, V. 27-Jun-1965, det. V., Nikolić, 43191 BEO, sub H. atomarium subsp. degenii, rev. B., Zlatković 31-Mar-2014); Grdelička klisura: Momin kamen, UTM EN82, 370 m, andezit, termofilne šume (leg. Đorđević, A. 2010, det. B., Zlatković, $7252 \mathrm{HMN}$ ); (leg. Tomović, G., Ranđelović, V., Zlatković, B. 07-Aug-1997, 8173 BEOU-KEGB, sub H. atomarium, rev. B., Zlatković 06-Feb-2014); Kukavica: Denčino - Vladimirovo, UTM EN73, hrastova šuma (leg. Jovanović, V. 1973, det. V., Jovanović, 5603 HMN); Srednja čuka, UTM EN73 (leg. Ilić, Đ., det. Đ., Ilić, 8698 BEO, sub H. atomarium, rev. B., Zlatković 31-Mar-2014); Radan: Petrova gora, UTM EN46, 1180 m, andezit, kamenjari, termofilne šume (leg. Zlatković, B., Ranđelović, v. 1995, 7255 HMN); Sokolov vis, Veliki krš, UTM EN35, silikat, stene (leg. Niketić, M., Tomović, G. 
01-May-2008, 26891 BEOU-KEGB, sub H. atomarium, rev. B., Zlatković 12-Dec-2013); Vranje, UTM EN71 (leg. Pančić, J., det. J., Pančić, 3349 BEOU (Herb. Panc.), sub H. atomarium, rev. B., Zlatković 06-Feb-2014); Dubnica, UTM EN60, kamenjar, 42521 BEOU); Leskovik, UTM EN71 (leg. Pančić, J., det. J., Pančić, 3348 BEOU (Herb. Panc.), sub H. atomarium, rev. B., Zlatković 06-Feb-2014); Markovo kale, UTM EN71 (leg. Pančić, J., det. J., Pančić, 3344 BEOU (Herb. Panc.), sub H. atomarium, rev. B., Zlatković 06-Feb-2014); Pljačkovica, UTM EN71, 428520, 42524 BEOU); (leg. Ilić, $Đ$. 1910, det. Đ., Ilić, 8695, 8697 BEO, sub H. atomarium, rev. B., Zlatković 31-Mar-2014); (leg. Ilić, V. 1910, det. Đ., Ilić, 8696 BEO, sub H. atomarium, rev. B., Zlatković 31-Mar-2014); Soderce, UTM EN71, kamenjar (leg. llić, Đ., det. Đ., Ilić, 8699 BEO, sub H. atomarium, rev. B., Zlatković 31-Mar2014); SE Serbia Pčinja - dolina: Jablanica, UTM EM78, $560 \mathrm{~m}$, biotit i gnajs, šume medunca i grabića, Querco-Carpinetum orientalis, 16269 BEOU); Surdulica, UTM EN92, silikat (leg. Niketić, M., Tomović, G., Zlatković, B. 04-May-2002, 15536 BEOU-KEGB, sub H. atomarium, rev. B., Zlatković 06Feb-2014); Trgovište, UTM EM88, hrastove šume, žbunovita mesta, kamenjari, 16269 BEOU); (leg. Tomović, G., Ranđelović, V., Zlatković, B. 08-Aug-1997, 8106 BEOU-KEGB, sub H. degenii, rev. B., Zlatković 06-Feb-2014); Vranje: Izumno, UTM EN81 (leg. Ničić, Đ. 28-Jul-1886, 42524 BEOU, sub H. atomarium, rev. B., Zlatković 12-Dec-2013); Vranjska Banja, UTM EN81 (leg. Dimitrijević, M. 6.1879, 42522 BEOU, sub H. atomarium, rev. B., Zlatković 12-Dec-2013); Vranjska Banja, UTM EN81 (leg. Pančić, J., det. J., Pančić, 3345 BEOU (Herb. Panc.), sub H. atomarium, rev. B., Zlatković 06-Feb-2014); Kosovo Šar-planina: Oštro koplje, UTM EN06 (leg. Soška, Th., 29-Jun-1914, 42505 BEOU); Metohija Šar-planina: Kobilica - Beograd, UTM DM86, 1650 m, kalkšisti, livada (leg. Duraki, Š., 06-Aug-2002, det. Š., Duraki, 975 BEOU-KEGB, sub H. perforatum, rev. B., Zlatković 06-Feb-2014); Kobilica - Poljane, UTM DM86, 1450 m, karbonat, hlorit-sericitski škrljici, planinski pašnjaci (leg. Duraki, Š. 16-Aug-2002, det. Š., Duraki, 974 BEOU-KEGB, sub H. maculatum, rev. B., Zlatković 06Feb-2014).

\section{Hypericum montanum L. (Fig. 18)}

NW Serbia Cer, UTM CQ74, CQ83, hrastova šuma, 7.1988, 42515 BEOU); obod šume, 1988, 45208 BEOU); hrastova šuma, 26-Jul-1989, 42623 BEOU); Divčibare, UTM DP18, serpentinit (leg. Stevanović, V. 10-Sep-1981, 2753 BEOU-KEGB); Krupanj: Vukulovac, UTM CQ61 (leg. Jurišić, ž. 06-Jun-1912, 8745 BEO); Loznica: Gućevo, podnožje, UTM CQ52 (leg. Jurišić, Ž. 03-Jun-1912, 8746 BEO); W Serbia Ovčarsko-Kablarska klisura: Kablar, UTM DP36 (leg. Jovanović, M., Niketić, M., 20-Jul-2004, 43330 BEO); Perućac, UTM CP76 (leg. Diklić, N., Nikolić, V. 13-Jul-1963, det. V., Nikolić, 43589 BEO, sub H. umbellatum, rev. B., Zlatković 31-Mar-2014); Derventa, UTM CP66, pored puteva (leg. Diklić, N., Nikolić, V., Rakin, M. 11-Jul-1963, det. N., Diklić, 43328 BEO); Derventa - kanjon, UTM CP66 (leg. Jurišić, Ž. 19-Jun-1912, 8749, 8750 BEO); Tara: Brusnica - kanjon, UTM CP66, krečnjak, stene, kamenjari (leg. Ostojić, D. 24-Jul-2001, 12442 BEOU-KEGB); Ivica, UTM CP75 (leg. Pančić, J., det. J., Pančić, 3378 BEOU (Herb. Panc.)); Kaluđerske bare, UTM CP86, šume, Ostrya carpinifolia (leg. Diklić, N. 30-Jun1965, det. N., Diklić, 43327 BEO); Miloše-

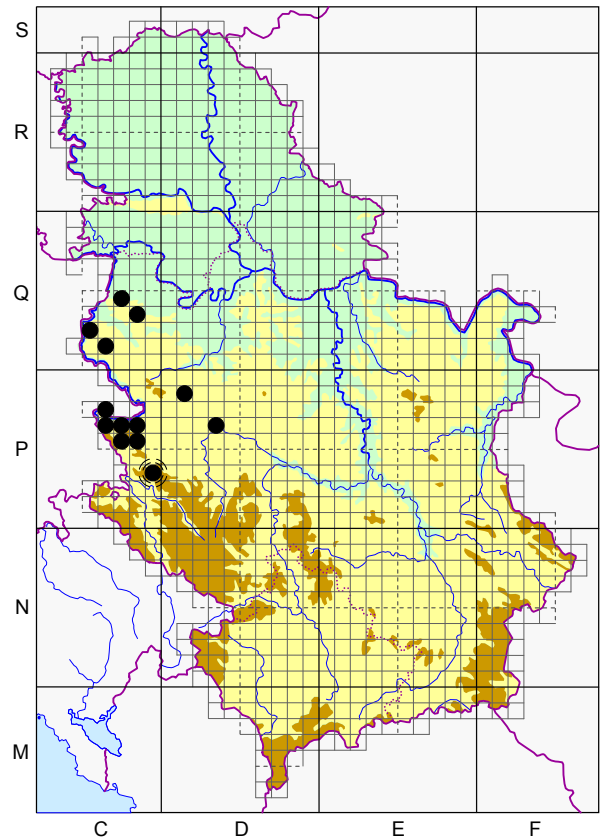

Fig. 18. - Distribution of the species H. montanum L. in Serbia. 
vac, UTM CP75 (leg. Pančić, J., det. J., Pančić, 3375 BEOU (Herb. Panc.)); Veliki Kraj, UTM CP67, krečnjak (leg. Stevanović, V., Vukojičić, S., Tomović, G., Zlatković, B. 02-Aug-2003, 17325 BEOU-KEGB); Užice: Mokra Gora, Jatare, UTM CP85 (leg. Jovanović, M. 19-Sep-2003, det. M., Jovanović, 43331 BEO); Zlatibor, UTM CP93 (leg. Pančić, J., det. J., Pančić, 3377 BEOU (Herb. Panc.)).

\section{Analysis of specimens and taxa}

The examination of all studied herbarium collections has shown presence of 1108 herbarium sheets with specimens of any taxa of the genus Hypericum collected within the territory of Serbia. Of this number, 426 specimens were deposited in collection of BEO, 484 specimens in collection of BEOU and 198 specimens in HMN. The surveys of herbarium material have shown evidence of presence of 18 accepted species from genus Hypericum, characterized by specific combinations of morphological characteristics. The misapplied name $H$. repens L. cited by Stjepanović-Veseličić (1972), was replaced with $H$. linarioides during the revision. Also, the cited $H$. richeri Vill., $H$. alpinum Waldst. \& Kit., non Vill and $H$. transsilvanicum Čelak. (Stjepanović-Veseličić 1972) actually belong to $H$. richeri subsp. grisebachii.

According to the number of specimens in inspected collections the representatives of $H$. sect. Drosocarpium (477) and $H$. sect. Hypericum (452) are best represented. To the contrary, the much smaller number of herbarium specimens was determined for the majority of sections: $H$. sect. Oligostema (1) H. sect. Hirtella (2), H. sect. Olympia (5), and $H$. sect. Androsaemum (6). The smallest number of herbarium sheets and UTM $(10 \times 10 \mathrm{~km})$ dots on the maps was recorded for the following species, which therefore should be treated as rare in Serbia: $H$. humifusum, $H$. hyssopifolium, $H$. androsaemum, $H$. linarioides and $H$. olympicum. At the level of individual taxa separately, $H$. perforatum L. subsp. perforatum was best-represented with 262 herbarium sheets. The poorest representation by herbarium data in collections was that of $H$. humifusum, recorded only in BEOU and represented by a single specimen. Complete survey and representation of revised herbarium data from three collections including all considered sections and taxa of Hypericum from Serbia is shown in Tab. 1.

\section{DISCUSSION AND CONCLUSIONS}

After the revision of herbarium material in BEO, BEOU and $\mathrm{HMN}$ it was concluded that $44.85 \%$ of the total number of exsiccates were unidentified or incorrectly identified. The survey and revision of specimens from the herbarium collections have resulted in a list of 18 species of genus Hypericum naturally occurring in the territory of Serbia.

The updated number of the species list regards several possible reasons. First of all, some important and reliable studies for genus Hypericum, largely 
Table 1. - Number of specimens of Hypericum species/subspecies deposited in $\mathrm{BEO}, \mathrm{BEOU}$ and $\mathrm{HMN}$.

\begin{tabular}{|c|c|c|c|c|c|c|}
\hline Taxon & BEO & $\begin{array}{c}\text { BEOU } \\
\text { (General) }\end{array}$ & $\begin{array}{c}\text { BEOU } \\
\text { (Herb Panc) }\end{array}$ & $\begin{array}{c}\text { BEOU } \\
\text { (KEGB) }\end{array}$ & HMN & Tota \\
\hline \multicolumn{7}{|c|}{ H. sect. Androsaemum } \\
\hline H. androsaemum & 2 & 3 & & 1 & & 6 \\
\hline \multicolumn{7}{|l|}{ H. sect. Hypericum } \\
\hline H. elegans & 6 & & 5 & & 2 & 13 \\
\hline $\begin{array}{l}\text { H. maculatum } \\
\text { subsp. maculatum }\end{array}$ & 6 & 2 & 3 & 14 & 11 & 36 \\
\hline $\begin{array}{l}\text { H. maculatum } \\
\text { subsp. immaculatum }\end{array}$ & 31 & 7 & 4 & 25 & 2 & 69 \\
\hline $\begin{array}{l}\text { H. perforatum } \\
\text { subsp. perforatum }\end{array}$ & 99 & 35 & 7 & 52 & 69 & 262 \\
\hline $\begin{array}{l}\text { H. perforatum } \\
\text { subsp. latifolium }\end{array}$ & 10 & 3 & & & & 13 \\
\hline $\begin{array}{l}\text { H. perforatum } \\
\text { subsp. veronense }\end{array}$ & 14 & 3 & 1 & 1 & 1 & 20 \\
\hline \multirow{2}{*}{$\begin{array}{l}\text { susp. verotrapterum } \\
\text { H. tetrat }\end{array}$} & 5 & 6 & 6 & 13 & 9 & 39 \\
\hline & 171 & 56 & 26 & 105 & 94 & 452 \\
\hline \multicolumn{7}{|l|}{ H. sect. Olympia } \\
\hline H. olympicum & 2 & & & 2 & 1 & 5 \\
\hline \multicolumn{7}{|l|}{ H. sect. Drosocarpium } \\
\hline H. barbatum & 47 & 24 & 8 & 29 & 7 & 115 \\
\hline H. montbretii & & 13 & & & & 13 \\
\hline $\begin{array}{l}\text { H. richeri } \\
\text { subsp. grisebachii }\end{array}$ & 36 & 15 & 7 & 15 & 6 & 79 \\
\hline H. rochelii & 45 & & 15 & 11 & 10 & 81 \\
\hline $\begin{array}{l}\text { H. rumeliacum } \\
\text { subsp. rumeliacum }\end{array}$ & 61 & 16 & 10 & 24 & 55 & 166 \\
\hline \multirow{2}{*}{ H. umbellatum } & 4 & 3 & 6 & 2 & 8 & 23 \\
\hline & 193 & 71 & 46 & 81 & 86 & 477 \\
\hline \multicolumn{7}{|l|}{ H. sect. Oligostema } \\
\hline $\begin{array}{l}\text { H. humifusum } \\
\text { subsp. humifusum }\end{array}$ & & & & 1 & & 1 \\
\hline \multicolumn{7}{|l|}{ H. sect. Hirtella } \\
\hline H. hyssopifolium & & & 1 & 1 & & 2 \\
\hline \multicolumn{7}{|c|}{ H. sect. Taeniocarpium } \\
\hline H. hirsutum & 37 & 9 & 8 & 25 & 12 & 91 \\
\hline \multirow[t]{2}{*}{ H. linarioides } & 3 & 2 & 2 & 1 & 2 & 10 \\
\hline & 40 & 11 & 10 & 26 & 14 & 101 \\
\hline \multicolumn{7}{|c|}{ H. sect. Adenosepalum } \\
\hline \multirow{3}{*}{$\begin{array}{l}\text { H. annulatum } \\
\text { subsp. annulatum } \\
\text { H. montanum }\end{array}$} & 11 & 14 & 8 & 10 & 3 & 46 \\
\hline & 9 & 3 & 3 & 3 & & 18 \\
\hline & 20 & 17 & 11 & 13 & 3 & 64 \\
\hline Total & 426 & 158 & 96 & 230 & 198 & 1108 \\
\hline
\end{tabular}


based on morphology, distribution, anatomy, cytology and phylogeny (Robson 1977, 1981, Nürk \& Blattner 2010), have been published after the paper by Stjepanović-Veseličić (1972). As the consequence, some of the taxa listed in "Flora of SR Serbia" have changed in nomenclature and taxonomic treatments.

The taxon H. boissieri Petrović was recognized by "Flora of SR Serbia" and some other relevant monographs from the region (Jordanov \& Kožuharov 1970) as a well-defined species, but according to the concept by Robson (1968) and Trinajstić (1975) it was considered only as a synonym for the variable species $H$. rochelii, distributed in Albania, Bulgaria, N.E. Greece, S.W. Romania and E. Serbia. However, H. boissieri (including $H$. boissieri var. latifolium Adamović), described from southeastern Serbia by Petrović (1885), may be distinguished from other similar taxa by several characters, first of all by presence of the intramarginal and superficial dark, and translucent glands across the surface of the leaves, as well as by shape of the vesicles on the ripe capsule (Jordanov \& Kožuharov 1970). On the other hand, according to Stjepanović-Veseličić (1972) H. rochelii was treated as a synonym of $H$. boissieri. It is interesting to note that the original description given by Petrović (1885) regards $H$. boissieri as more similar to $H$. spruneri Boiss. (which does not grow in Serbia) than to $H$. rochelii also represented in Serbia according to the same author (Petrović $1882,1885)$. Even the type specimen of $H$. boissieri was not found neither in BEO nor in BEOU, all the samples identified under that name fall into the prominent variability of $H$. rochelii according to our revision. The relationship of $H$. rochelii, $H$. spruneri and $H$. boissieri certainly deserves more attention and additional field research, which would help solving the taxonomic problematic within this interesting group of taxa.

A specific problem in identification was posed by the particularly high variability of morphological characters in some Hypericum species, resulting in their incorrect identification, documented in large percent of the cases in collections. One good example for this problem again pertains to several taxa of $H$. sect. Drosocarpium. Majority of the species from this group, e.g. $H$. barbatum, $H$. richeri, $H$. rochelii and $H$. rumeliacum are characterized by prominent variability and plasticity of morphological characters, especially their habit. $H$. rochelii is particularly variable in length and shape of the cauline leaves. Several infraspecific taxa were described from the neighboring regions (Gusuleac \& Nyarady 1956, Jordanov \& Kožuharov 1970), additionally making difficult determination and separation of this group from other representatives of the section. It was proven that a large number of specimens of $H$. rochelii were wrongly identified, not only as $H$. boissieri, but also as $H$. rumeliacum, $H$. barbatum and $H$. richeri, and vice versa. 
As noted, $H$. alpinum Waldst. \& Kit., non Vill. and $H$. transsilvanicum Člak., both synonyms of $H$. richeri subsp. grisebachii, were considered as separate species in the edition "Flora of SR Serbia" (Stjepanović-Veseličić 1972, 1977, Diklić 1986), creating additional confusion for the reader. It remains an open question if enumeration of $H$. richerii with no infraspecific taxa added by Stjepanović-Veseličić (1972) refers to H. richeri subsp. richeri as its presence was not recorded in revised herbarium material from Serbia.

The situation was similar in $H$. sect. Hypericum, as its representatives are characterized by a large variation in morphological traits (Robson 1986, 2002). Due to the prominent variability of infraspecific taxa of $H$. perforatum it was difficult to distinguish between the taxa treated at the rank of subspecies (H. p. subsp. perforatum, H. p. subsp. latifolium and $H$. p. subsp. veronense) for this purpose. This taxonomic complex of $H$. perforatum s.l., as one of the best-known member of the genus, certainly deserves further taxonomic and nomenclature reconsideration.

It should also be notified that Stjepanović-Veseličić (1972) includes species $H$. repens, which was probably misapplied by $H$. linarioides. The first record for the doubtful presence of species $H$. repens in Serbia was published by Pančić (1884), and it was probably based on few voucher specimens deposited in collection "Herbarium Pancicianum" (BEOU), that were checked and identified as $H$. linarioides during the recent revision.

\section{Acknowledgements}

The authors would like to thank our colleagues Snežana Vukojičić, Marija Marković and Uroš Buzurović who kindly helped with work in herbarium collections, as well as Gordana Tomović for helpful guidance. The Ministry of Education, Science and Technological Development of the Republic of Serbia supported this research through Grant 173030 "Plant biodiversity of Serbia and the Balkans - assessment, sustainable use and protection".

\section{REFERENCES}

Crockett, S. L., Robson, N. K. B. (2011): Taxonomy and Chemotaxonomy of the Genus Hypericum. - Medicinal and Aromatic Plant Science and Biotechnology 5(1): 1-13.

Diklić, N. (1986): Hypericaceae Lindl. In: Sarić, M. R. (ed.): Flora SR Srbije 10: 58. - Srpska Akademija nauka i umetnosti, Odeljenje prirodno-matematičkih nauka, Beograd. [In Serbian]

Gusuleac, M., Nyarady, E. I. (1956): Hypericaceae Lindl. In: Savulescu, T. (ed.): Flora Reipublicae Popularis Romanicae 4: 25-46. - Editura Academiei Republicii Populare Romine, Bucuresti. [In Romanian] 
Jordanov, D., Kožuharov, S. (1970): Guttiferae Juss. In: Jordanov, D. (ed.): Flora na Narodna Republika B'lgaria 4: 224-273. - B'lgarskata Akademija na Naukite, Sofija. [In Bulgarian]

Lampinen R. (2001): Universal Transverse Mercator (UTM) and Military Grid Reference System (MGRS). [Available at: http://www.luomus.fi/english/botany/ afe/map/utm.htm]

Micevski, K. (1995): The Flora of the Republic of Macedonia 1(3). - Macedonian Academy of Sciencec and Arts, Skopje. [In Macedonian]

Nürk, N. M., Blattner, F. R. (2010): Cladistic analysis of morphological characters in Hypericum (Hypericaceae). - Taxon 59: 1495-1507.

Pančić, J. (1884): Dodatak flori kneževine Srbije. - Kraljevsko-srpska državna štamparija, Beograd. [In Serbian]

Petrović, S. (1882): Flora okoline Niša. - Kraljevsko-srpska državna štamparija, Beograd. [In Serbian]

Petrović, S. (1885): Dodatak flori okoline Niša. - Kraljevsko-srpska državna štamparija, Beograd. [In Serbian]

Robson, N. K. B. (1968): Hypericum L. In: Tutin, T. G., Heywood, V. H., Burges, N. A., Moore, D. M., Valentine, D. H., Walters, S. M., Webb, D. A. (ed.): Flora Europaea 2: 261-296. - University Press, Cambridge

Robson, N. K. B. (1977): Studies in the genus Hypericum L. (Guttiferae) 1. Infrageneric classification. - Bulletin of the British Museum (Natural History), Botany 5: 291-355.

Robson, N. K. B. (1981): Studies in the genus Hypericum L. (Guttiferae): 2. Characters of the genus. - Bulletin of the British Museum (Natural History), Botany 8: 55-226.

Robson, N. K. B. (2002): Studies in the genus Hypericum L. (Guttiferae) 4(2). Section 9. Hypericum sensu lato (part 2): subsection 1. Hypericum series 1. Hypericum. - Bulletin of the British Museum (Natural History), Botany 32(2): 61-123.

Stevens, P. F. (2007): Hypericaceae L. In: Kubitzki, K. (ed.): The families and genera of vascular plants (Flowering Plants, Eudicots) 9: 194-201. - Springer-Verlag, Berlin-Heidelberg. 9

Stjepanović-Veseličić, L. (1972): Hypericaceae Lindl. In: Josifović, M. (ed.): Flora SR Srbije 3: 104-125. - Srpska Akademija nauka i umetnosti, Odeljenje prirodno-matematičkih nauka, Beograd. [In Serbian]

Stjepanović-Veseličić, L. (1977): Hypericaceae Lindl. In: Josifović, M. (ed.): Flora SR Srbije 9: 70. - Srpska Akademija nauka i umetnosti, Odeljenje prirodno-matematičkih nauka, Beograd. [In Serbian]

Takhtajan, A. (2009): Flowering Plants, 2nd edn. - Springer Science \& Business Media B.V., Dordrecht.

The EURO+MED Plantbase - the information resource for Euro-Mediterranean plant diversity. [http://ww2.bgbm.org/EuroPlusMed/]

Thiers, B. (2014): Index Herbariorum: A global directory of public herbaria and associated staff. - New York Botanical Garden's Virtual Herbarium. [continuously updated: http://sweetgum.nybg.org/ih]

Trinajstić, I. (1975): Hypericum L. In: Trinajstić, I. (ed.): Analitička flora Jugoslavije 2(1): 9-30. - Institut za botaniku Sveučilišta u Zagrebu, Zagreb. [In Serbo-Croatian]

Zlatković, B., Tomović. G., Ranđelović, V., Vukojičić, S., Niketić, M. (2009): Distribution and conservation status of several new and neglected vascular plants in Serbia. - Phytologia Balcanica 15(1): 95-105. 


\title{
РЕВИЗИJA РОДА НYPERICUM L. (HYPERICACEAE) У TPИ ХЕРБАРИЈУМСКЕ ЗБИРКЕ ИЗ СРБИЈЕ
}

\author{
БОЈАН ЗЛАТКОВИЋ, МАРКО НИКОЛИЋ, МИЉАНА ДРНДАРЕВИЋ, \\ МИРОСЛАВ ЈОВАНОВИЋ, МАРЈАН НИКЕТИЋ
}

\section{Р Е 3 И М Е}

Рад представља каталошки приказ хербарских примерака за 18 врста рода Hypericum L. сакупљених на подручју Србије, регистрованих прегледом три хербаријумске колекције: Хербаријума Природњачког музеја у Београду (ВEО), Хербаријума Института за ботанику и Ботаничке баште "Јевремовац", Универзитета у Београду (BEOU) и Хербаријума Природно-Математичког факултета, Универзитета у Нишу (HNM). Прегледано је укупно 1108 ексиката, при чему се 426 примерака чува у BEO, 484 у BEOU и 198 у збирци HNM. На приложеним картама је за сваку испитивану врсту приказана дистрибуција базирана на провереним хербаријумским подацима о њеном распрострањењу у Србији. 